\title{
FLUID FLOW CHARACTERIZATION IN RAPID PROTOTYPED COMMON ILIAC ARTERY ANEURYSM MOLDS
}

\author{
A Thesis \\ presented to \\ the Faculty of California Polytechnic State University, \\ San Luis Obispo
}

\author{
In Partial Fulfillment \\ of the Requirements for the Degree \\ Master of Science in Biomedical Engineering
}

by

Daniel Cole Greinke

March 2016 
(C) 2016

Daniel Cole Greinke

ALL RIGHTS RESERVED 
COMMITTEE MEMBERSHIP

TITLE: $\quad$ Fluid Flow Characterization in Rapid Prototyped

Common Iliac Artery Aneurysm Molds

AUTHOR: Daniel Cole Greinke

DATE SUBMITTED: $\quad$ March 2016

COMMITTEE CHAIR: $\quad$ David Clague, Ph.D.

Professor of Biomedical Engineering

COMMITTEE MEMBER: Kathy Chen, Ph.D.

Professor of Biomedical Engineering

COMMITTEE MEMBER: $\quad$ Richard Savage, Ph.D.

Professor of Biomedical Engineering 


\begin{abstract}
Fluid Flow Characterization in Rapid Prototyped Common Iliac Artery Aneurysm Molds

Daniel Cole Greinke
\end{abstract}

The goal of this project was to determine whether i) fused deposition modeling could be employed to manufacture molds for vascular constructs, ii) whether vascular constructs could be created from these molds, and iii) to verify practical equivalence between observed fluid velocities. Dye tracking was to be employed to characterize fluid velocity profiles through the in vitro vascular constructs, including a half-vessel model and a full vessel model of an iliac artery aneurysm. A PDMS half-vessel construct was manufactured, and the movement of dye through the construct was tracked by a cellphone camera. Thresholds were applied to each video in HSB or YUV mode in ImageJ, and analyzed to determine the velocity of the fluid through the construct. COMSOL simulations of the half-vessel were conducted for comparison to the empirical observations. Plots describing the flow velocities along the maximum streamline path length were generated, and a one sample t-test was conducted at a 5\% significance level to determine whether there was a significant difference between velocity values obtained by dye tracking and the COMSOL simulations. It was determined that the empirical dye tracking trials failed to demonstrate agreement between the measured and predicted flow rates. A full vessel construct was not completed due to unforeseen time constraints.

Dye tracking was not determined to be reliable as a means of measuring the maximum velocity of fluid. Discrepancies between the empirical observations and the 
COMSOL simulation are discussed. The discrepancy was attributed to limitations in the experimental protocol; low frame rate, poor control over lighting conditions, and the subjectivity involved in image processing. Methods of improving upon the manufacturing and experimental protocols used for the half-vessel are proposed for future work, such as improving control over lighting conditions, choosing a camera with a higher frame rate, constructing a more stable fixture, exploring PIV. Additionally, the technical problems leading to the failure to complete the full vessel model are discussed, and changes in the manufacturing process are proposed to allow dissolution or removal of the aneurysm model.

Keywords: Iliac Artery Aneurysm, Hemodynamics, Dye Tracking, COMSOL, Flow Visualization 


\section{ACKNOWLEDGMENTS}

I would like to thank my parents, Gary and Karen for their support as I pursued a Master's degree in Biomedical Engineering.

I thank my significant other, Rebecca McGillivary, for the much needed emotional support throughout this project. I promise to reciprocate as she pursues her Ph.D. with the UCSF Tetrad program.

Thanks to Dr. Clague for welcoming me into the research group, for the feedback on my work over the past year, and for the help obtaining materials for the project.

Thanks to Rachel Willis for her contributions to our experimental setup and data analysis, and for multiple drives to the hardware store for materials.

Thanks to Dr. Richard Savage for granting Rachel and I access to the clean room for the plasma treatments, and for advice regarding the heat treatment.

Thanks to Dr. Andrew Schaffner for his advice regarding statistical analysis for this project.

Thanks to Dave Laiho for his help with printing the half vessel aneurysm model. 


\section{TABLE OF CONTENTS}

Page

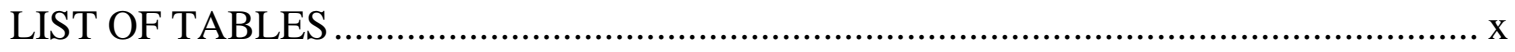

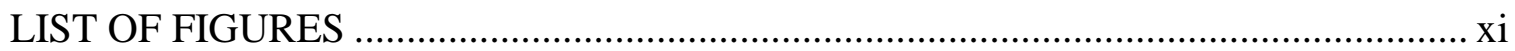

\section{CHAPTER}

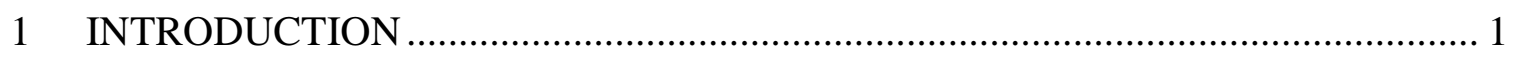

1.1 Specific Goals of this Thesis Project and Report............................................... 1

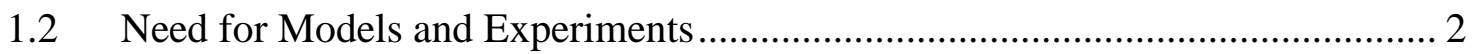

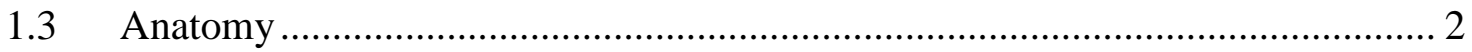

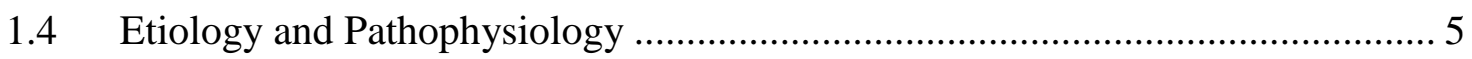

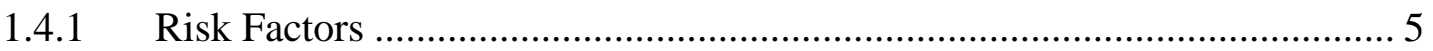

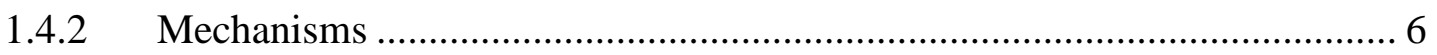

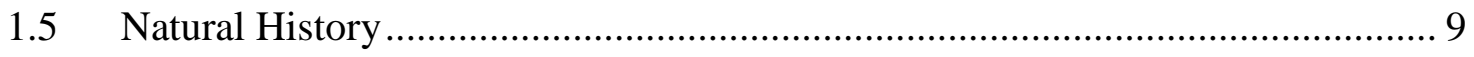

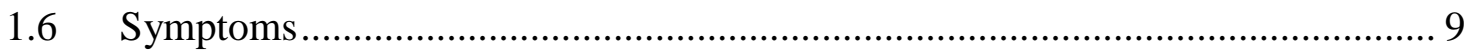

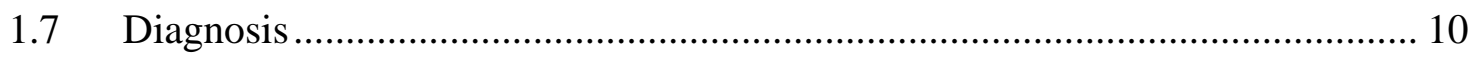

1.7.1 Ultrasonography ................................................................................ 13

1.7.2 Computed Tomography ...................................................................... 14

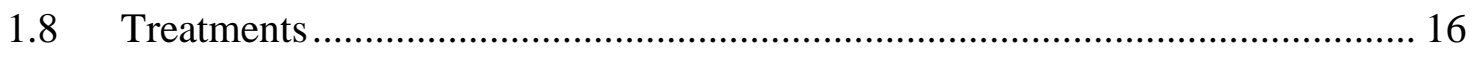

1.8.1 Endovascular Repair ........................................................................ 16

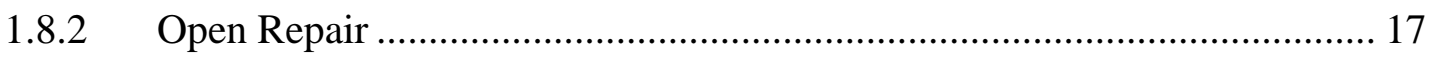

1.9 Previous Research into Aneurysmal Hemodynamics ........................................ 18

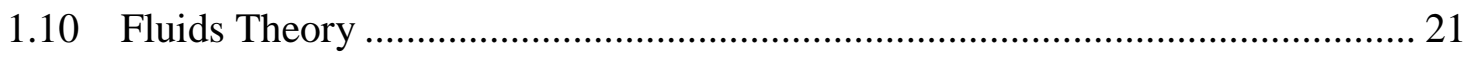

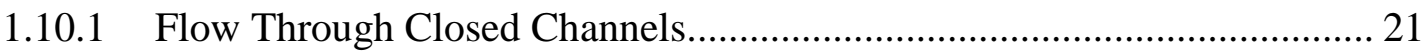


1.10.2 Fluid Properties ................................................................................... 24

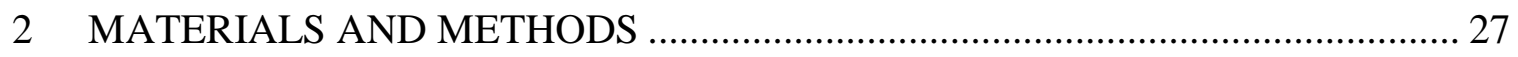

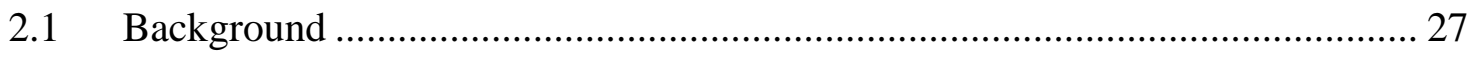

2.1.1 Fused Deposition Modeling ..................................................................... 27

2.1.2 Model Preparation............................................................................... 28

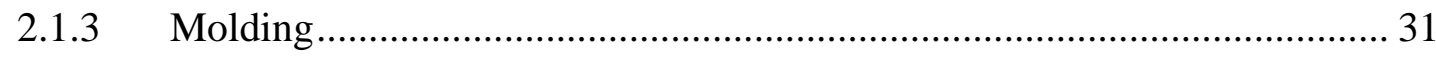

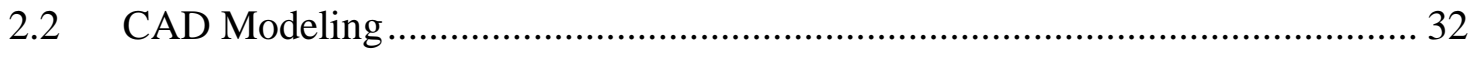

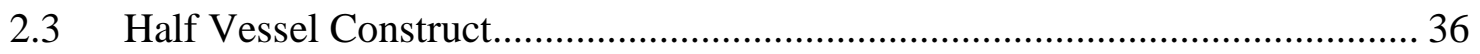

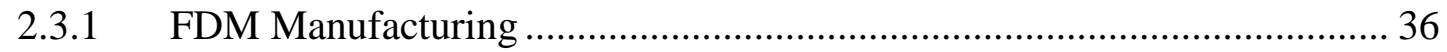

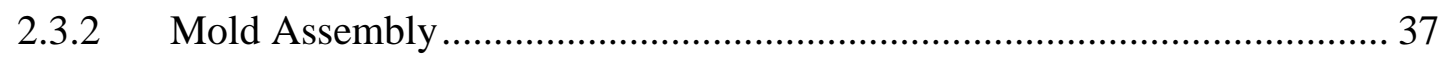

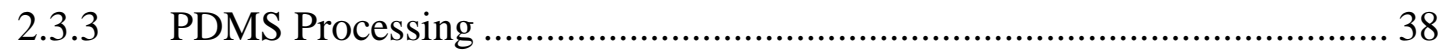

2.3.4 Aneurysm Construct Manufacturing ........................................................ 39

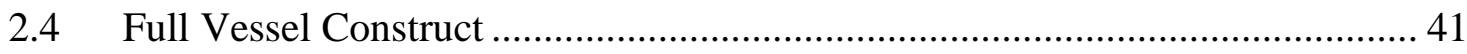

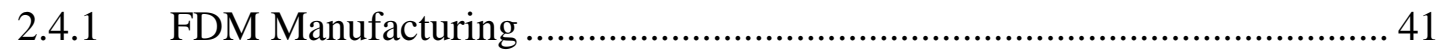

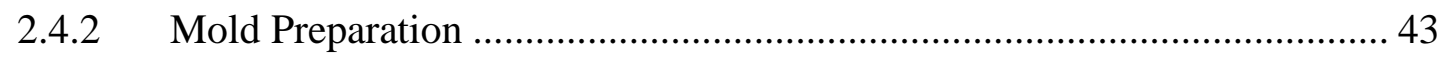

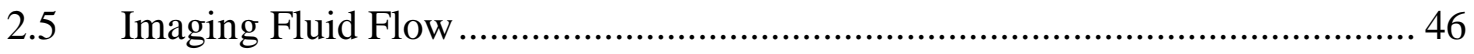

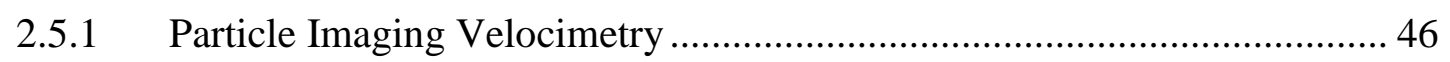

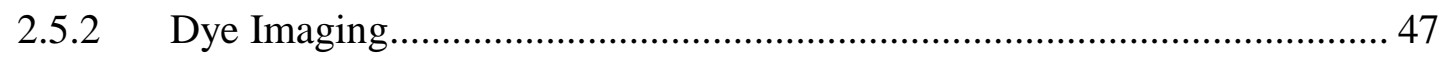

2.5.3 Image Processing …………………………….................................... 50

2.5.4 Image Analysis............................................................................ 51

2.6 Half Vessel COMSOL Validation................................................................... 54

2.6.1 Data Processing and Analysis ............................................................. 55

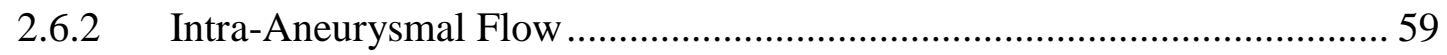




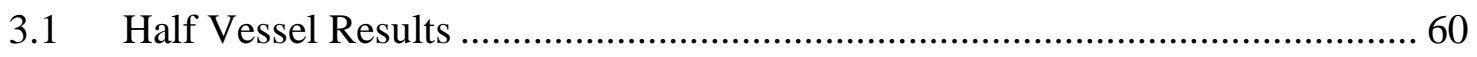

3.1.1 Comparison of Empirical Velocity Measurements to COMSOL ............... 60

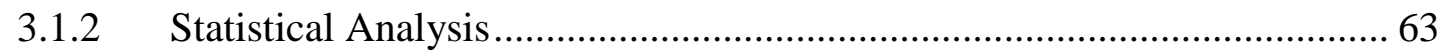

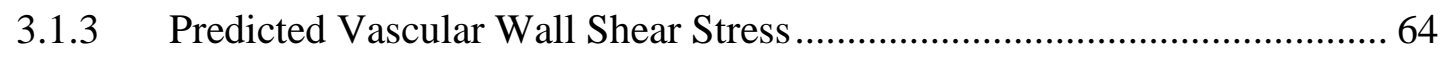

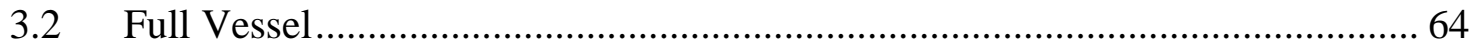

3.3 Discussion of Half Vessel Findings ......................................................... 65

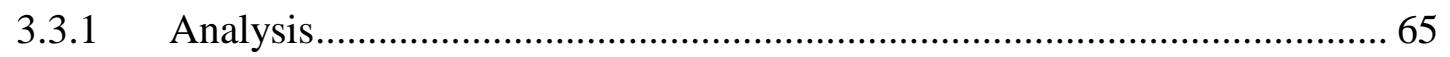

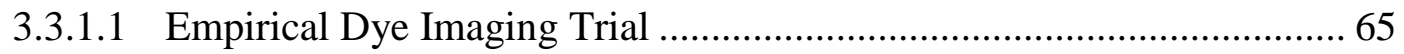

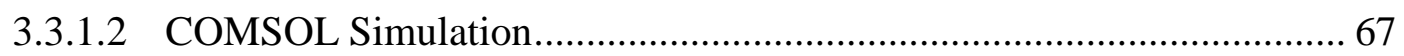

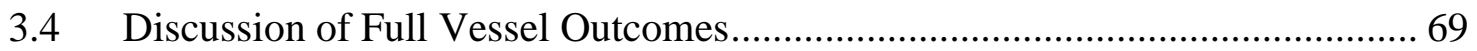

4 SUMMARY AND CONCLUSIONS ........................................................... 71

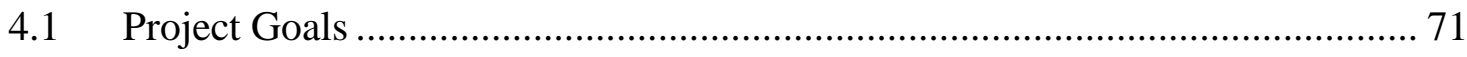

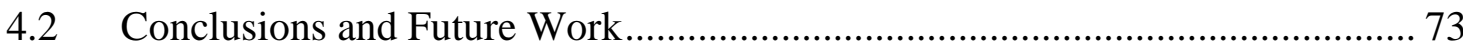

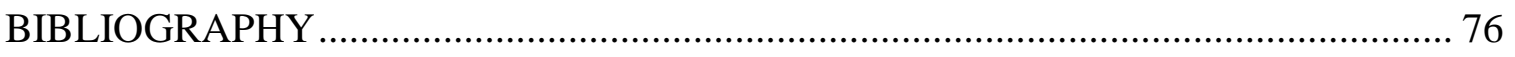
APPENDICES

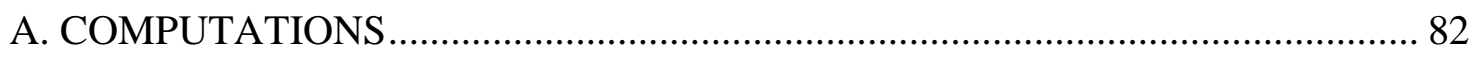

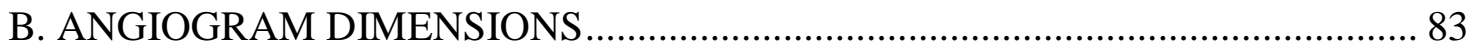

C. ANEURYSM MODEL DESIGN .............................................................. 84

D. FRAMES OF HALF VESSEL IMAGING TRIAL AFTER THRESHOLD.......... 87 


\section{LIST OF TABLES}

Table

Page

Table 1: Aneurysm Management According to Size ................................................. 11

Table 2: Flow Regime According To Reynolds Number .......................................... 23

Table 3: Half Vessel Mesh Statistics ..................................................................... 55

Table 4: Changes to COMSOL Solution with Increasing Mesh Fineness....................... 68

Table 5: Arterial Diameter Measurements Extracted from Angiogram. .......................... 83 


\section{LIST OF FIGURES}

Figure

Page

Figure 1: Gross anatomy of the vasculature of interest including the Abdominal Aorta, the common iliac arteries downstream and the internal iliac arteries and external iliac arteries [25]

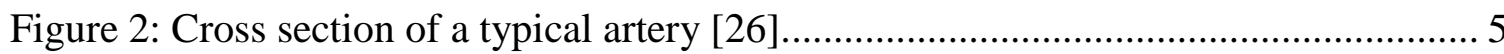

Figure 3: Visual representation of likely interplay of biological and hemodynamic

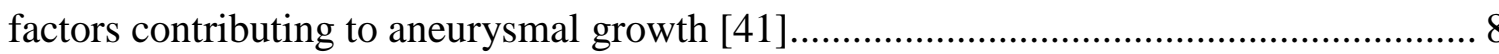

Figure 4: As the shear rate increases, the apparent viscosity of blood decreases and approaches an asymptote at $3.0 \mathrm{cP}$. Therefore, blood is considered to be a shear thinning fluid which can be treated as Newtonian at shear rates higher than $150 \mathrm{sec}-1$ [38].

Figure 5: Schematic of FDM operation. A 3D model is split into 2D cross sections which are added drawn by an extruder layer-by-layer to produce a 3D object.

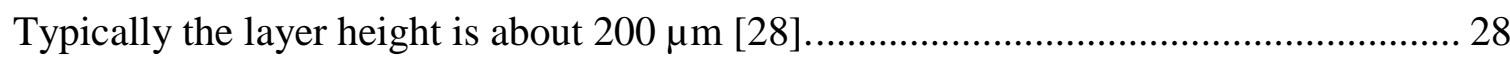

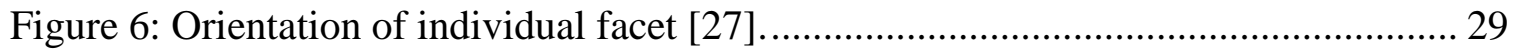
Figure 7: Vertex-to-vertex rule requires vertex coordinates to be shared between facets, prevents vertices from meeting side of facet [27]. 30 Figure 8: Abdominal angiogram of a patient suffering fibromuscular dysplasia, a condition unrelated to aneurysmal disease. This condition resulted in beading shown on the external iliac arteries, but did not affect the overall arterial dimensions [44]. 34 Figure 9: To ensure the half-vessel model could be printed, the model was split into multiple pieces with pegs and holes added, and arranged to ensure all pieces would fit 
the uPrint build surface. At this point, the conical connector had not been added, which is why the aortic section was modified and reprinted in PLA on the Series 1 at a later date.

Figure 10: Aneurysm model after FDM manufacturing. The aneurysm model was assembled and used in the molding process to define the shape of the channel of the in vitro model. The aortic section was modified to add the conical connector with a 3/16 inch diameter peg. 36

Figure 11: Completed mold featuring plexiglass walls fixed to a glass surface with caulk. This configuration allowed the PDMS mold to set before being fixed to the glass by plasma bonding.

Figure 12: The general steps in construct production include a) construction of the mold, and pouring PDMS, b) allowing the PDMS to set, c) separating the PDMS from the glass slide and plexiglass walls, d) removal of aneurysm model, and cleaning mold, followed by a plasma treatment, leaving e) the final aneurysm construct which was subsequently subjected to a heat treatment described below.

Figure 13: Manufacturing the aneurysm model, and producing a mold resulted in four types of artifacts, including a) ridges in the PDMS left by the edges of individual layers from the FDM process, b) infill of the joints between components, leaving a flap which needed to be removed by trimming, c) excess PDMS filling the gap between the model outlets and the mold wall; which required removal to allow flow through the mold, and d) excess PDMS flaps underneath the halfvessel model which was removed prior to flow visualization. 
Figure 14: "Cornucopia Connector" after printing had finished. The print featured support material to prevent warping during print. Support material was removed before the molding process.

Figure 15: Full Vessel Mold half filled with PDMS. At this stage, the PDMS was allowed to set so the model would be secured in place, preventing bouyant forces from lifting the model out of the mold once the model was completely submerged in the subsequent layer.

Figure 16: Full Vessel construct after PDMS had set, and plexiglass sides were removed 45

Figure 17: The general steps involved in the production of the full vessel include a) mold assembly and pouring half the PDMS into the mold, b) allowing the PDMS to set, c) pouring in the second half of the PDMS, d) allowing the final layer of PDMS to set, e) cutting excess material away from the outlets, and f) dissolving the PVA by submerging the mold in hot water. 46

Figure 18: Schematic of imaging setup. Clear water was pumped through the construct until a flow profile was established. Next, dye was introduced to the inlet and it's flow patterns were captured by the camera before draining into a sink.

Figure 19: Once the coordinates of the dye fronts were recorded, the scaling was adjusted to convert pixels to meters. However, it was necessary to convert these positions into the COMSOL coordinate system to generate a streamline model, which was used to select point evaluations in COMSOL that would correspond to the motion of the dye in the images. 
Figure 20: The coordinate transformation converted the recorded dye front positions into the coordinate system used in COMSOL. However, Run 5 (dark blue) was dropped for the purposes of creating the streamline model, because a translational error which shifted the data points in the positive y direction, outside the aneurysm model in COMSOL. Data from Run 5 was still used to obtain velocity measurements which should not have been affected by the translational error. 53 Figure 21: A schematic of the data acquisition techniques used to extract velocity measurements from the dye imaging trials. 53 Figure 22: A sixth order polynomial model of the maximum velocity streamline was generated based on the positions of the dye fronts in the images. This model was used to generate point evaluations of the fluid velocity within the COMSOL model of the aneurysm. The function exhibited undesired curvature for z-values less than -0.213 , located within the aorta. These point evaluations were omitted from an analysis of the velocity profile. 58

Figure 23: A comparison between the velocity measurements output by COMSOL and empirical measurements. A discrepancy was evident based on a cursory examination of the data. However, the ratio of the area between each empirical plot and the COMSOL plot was generated using trapezoidal rule and identical limits for each comparison were used to obtain a quantitative assessment of this discrepancy

Figure 24: An arrow plot using logarithmic scaling shows the presence of a high velocity jet at the medial surface, and low velocity back flow at the lateral side of the aneurysm, demonstrating rotational flow within the aneurysm. 
Figure 25: Surface Plot of velocity provides quantitative assessment of intraaneurysmal velocity profile, revealing the high velocity jet at the medial surface of the aneurysm.

Figure 26: Overlay of velocity surface plot and point evaluations generated in COMSOL, including both the streamline model and the raw data used to generate that model. Roughly the distal half of the streamline model runs slightly medial to the maximum velocity jet through the aneurysm predicted by COMSOL

Figure 27: Shear stress slice plot within aneurysm model. The highest shear stress regions are located at the bifurcations of the vasculature.

Figure 28: The aneurysm model was constructed from a series of splines with points placed at semi-regular intervals along the splines. 84

Figure 29: Circles at desired dimensions were centered on the chosen points, perpendicular to the spline. Next, a loft was applied to connect the circles and obtain the organic form of vasculature. 85

Figure 30: The geometry was exported to COMSOL, cut in half along the x-z plane. The COMSOL simulation did not include the connector used in the dye tracking study, assuming a uniform flow field at the inlet, leading to a discrepancy in the boundary conditions 86

Figure 31: In preparation for FDM manufacturing, a $3 \mathrm{~cm}$ conical connector was added to the end of the half vessel model, and the EIA's and IIA's were trimmed to

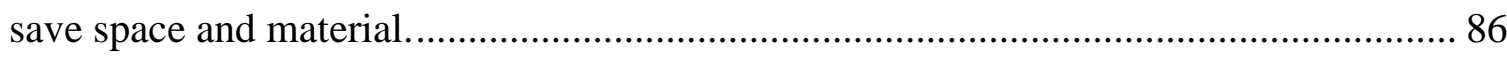

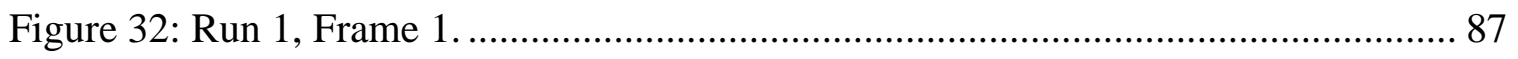

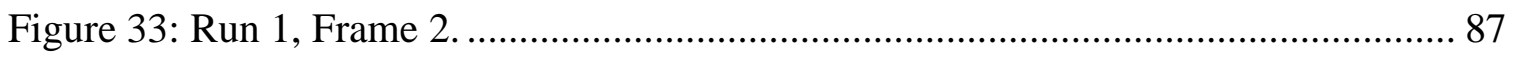




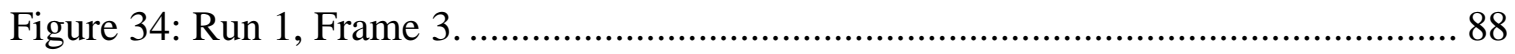

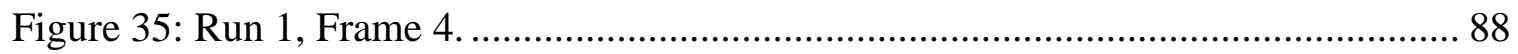

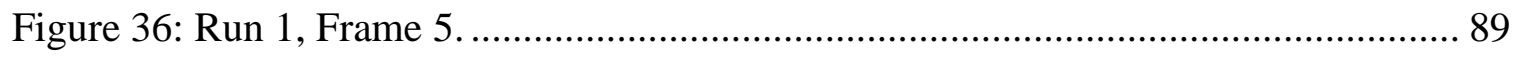

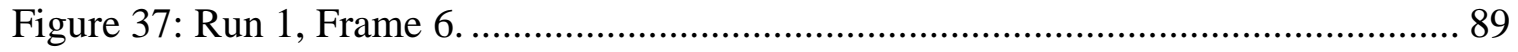

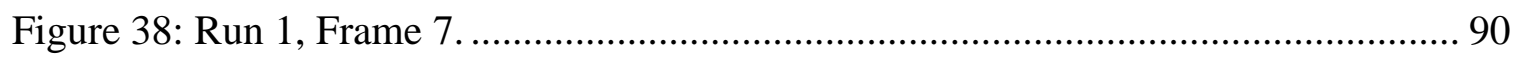

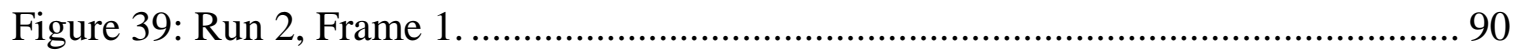

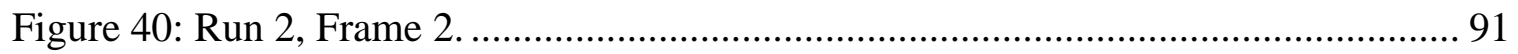

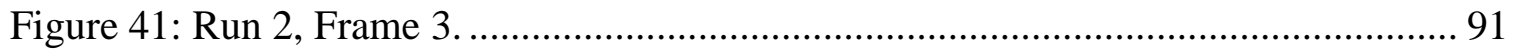

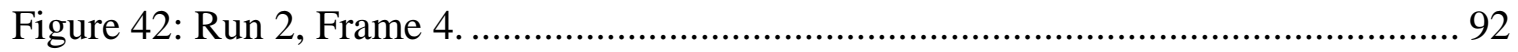

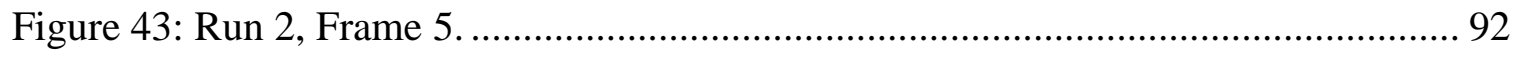

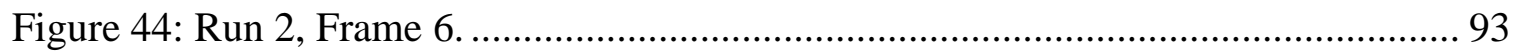

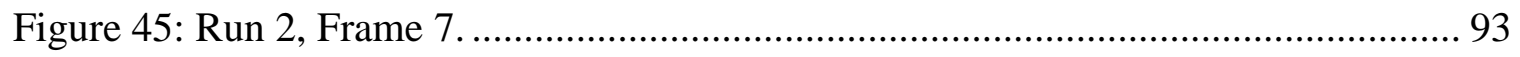

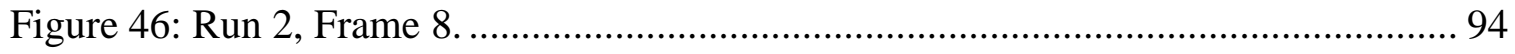

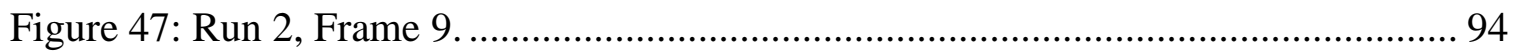

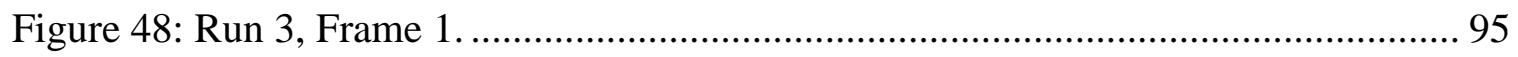

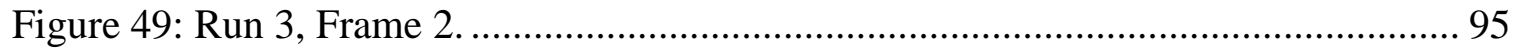

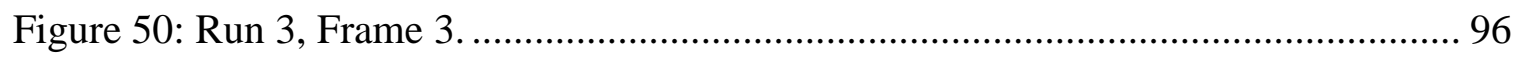

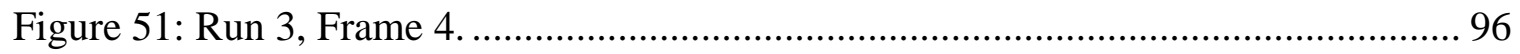

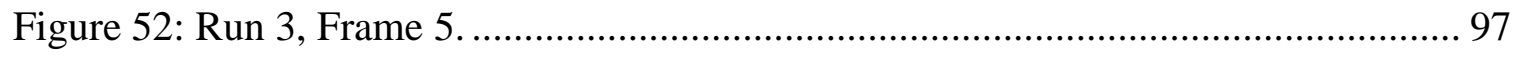

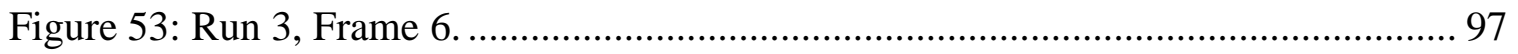

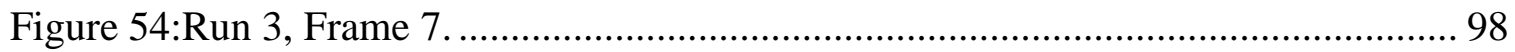

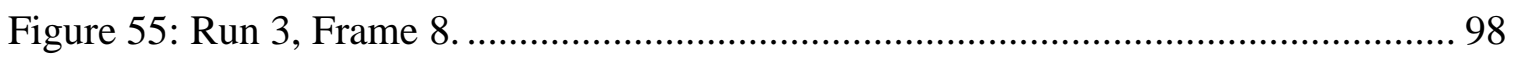

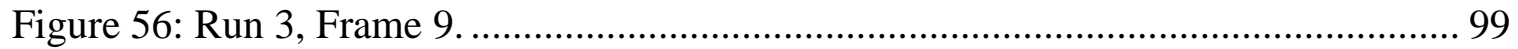




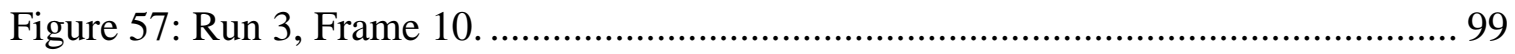

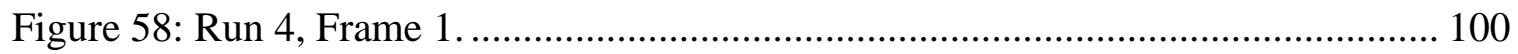

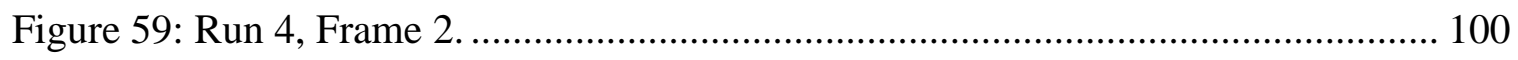

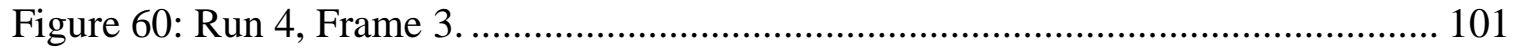

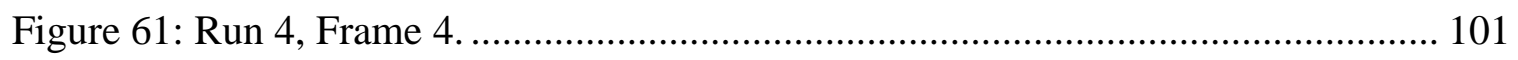

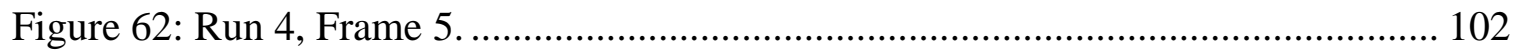

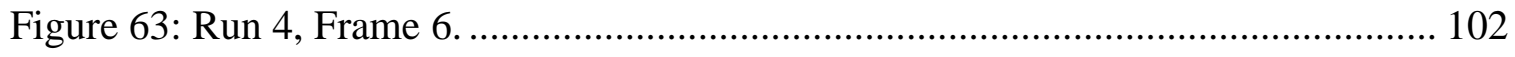

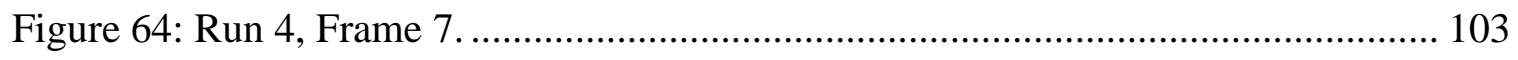

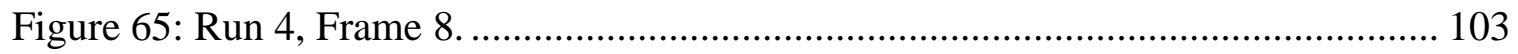

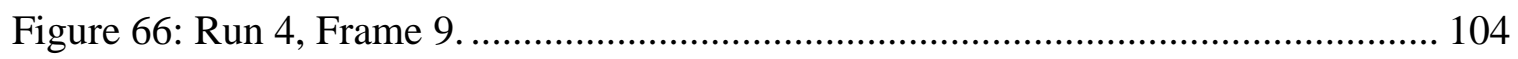

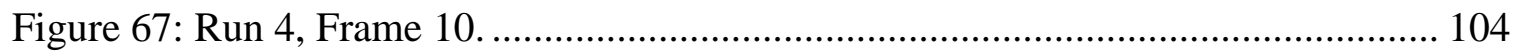

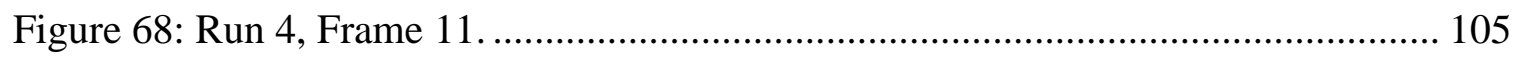

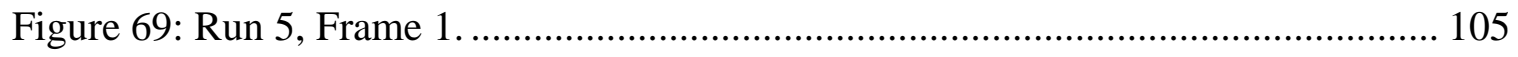

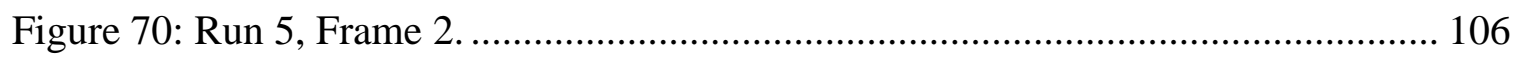

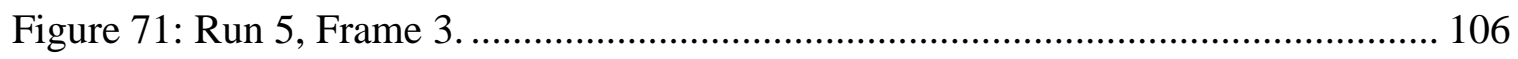

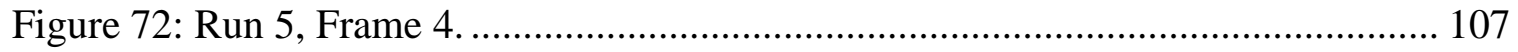

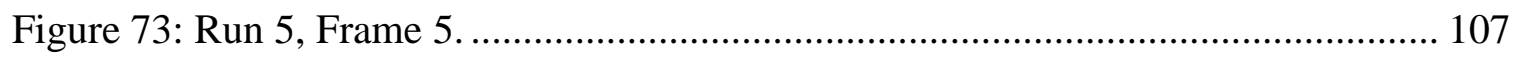

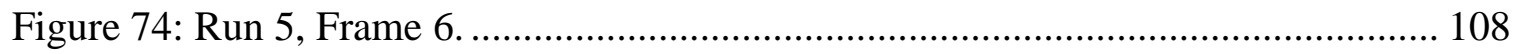

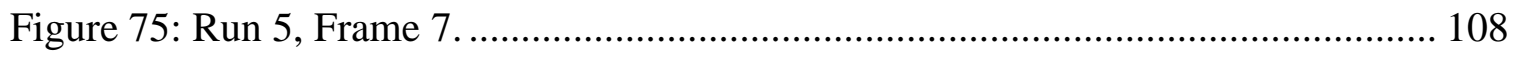

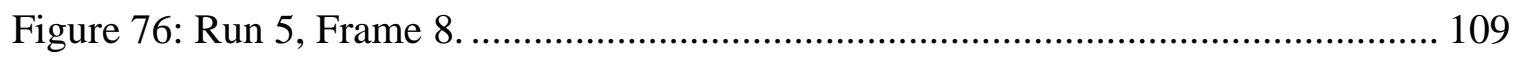

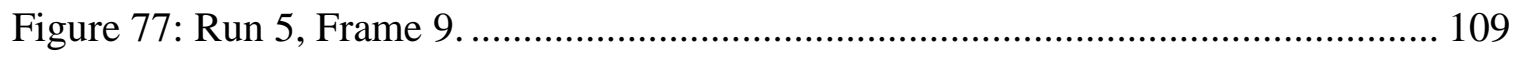

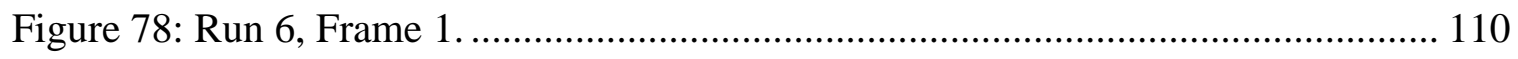

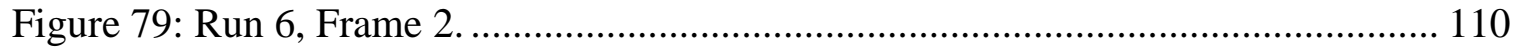




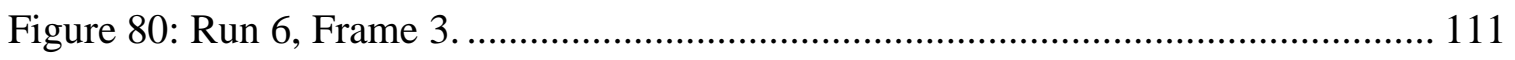

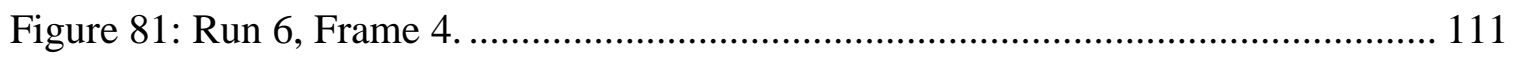

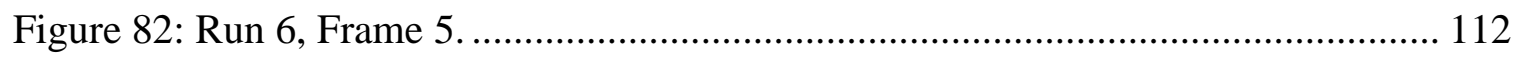

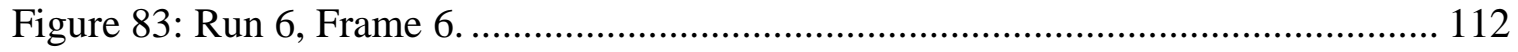

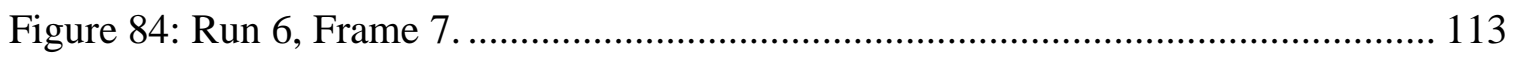

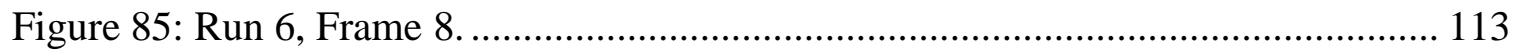

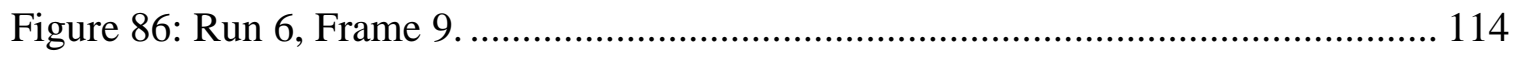




\section{INTRODUCTION}

\subsection{Specific Goals of this Thesis Project and Report}

The goal of this project was to make a three dimensional construct of an isolated common iliac artery aneurysm and to validate a complementary predictive COMSOL model of blood flow conditions in an iliac artery aneurysm. The experiments conducted as a part of this thesis project were intended to quantify fluid flow rates through aneurysm molds which were identical to the models used in COMSOL computer simulations. Two models were constructed; a half vessel model which featured a plane cutting longitudinally though the center of the model, and a full three dimensional vessel construct. The half vessel construct featured a no-slip condition at the glass surface which functioned as a "window" into the blood vessel for fluid flow visualization. In the full vessel construct, there was no glass surface and therefore it lacked a no-slip condition at the center of the construct. Success was defined by the ability of the experiments to validate the fluid flow conditions predicted in COMSOL simulations of the half vessel and the full vessel constructs. In conducting these studies, this thesis sought to answer the following questions:

1. Can fused deposition modeling be used to construct half vessel and full vessel constructs of an iliac artery aneurysm?

2. Can the velocity profile through the aneurysm models be measured experimentally using dye?

3. Was there good agreement between COMSOL and fluid flow visualization studies? 


\subsection{Need for Models and Experiments}

The purpose of characterizing the fluid velocity profiles through an aneurysm construct was to provide empirical verification of the predicted velocity profile that was output by the COMSOL simulation, and allow corrections to be made to the simulation accordingly. The shear stress along the vascular walls could not be measured directly by empirical studies, but it could be predicted with the aid of a COMSOL simulation. An understanding of the shear stress conditions within an iliac artery aneurysm would allow predictions of the effects of hemodynamic phenomena on the natural history of iliac artery aneurysms.

\subsection{Anatomy}

Blood pumped from the heart travels through the aorta, the largest artery in the human body, with a diameter of about $2.5 \mathrm{~cm}$. The aorta starts at the superior aspect of the left ventricle of the heart, forming the aortic arch which branches off to form the subclavian arteries and carotid arteries. Past the arch, the thoracic aorta extends through the thoracic cavity, branching off to supply blood to structures there. Finally, the abdominal aorta extends from the inferior aspect of the diaphragm, branching off to the renal arteries and extending down to the level of the fourth lumbar vertebra and splits off into the two

common iliac arteries (Figure 1) [7]. The common iliac arteries (with a diameter of about $1.25 \mathrm{~cm}$ ) run laterally along the medial edge of the psoas muscle and branch off into the internal iliac arteries and the external iliac arteries anterior to the sacroiliac joint. The internal iliac artery extends through the true pelvis to the superior aspect of the greater 
sciatic foramen. The external iliac artery travels along the medial border of the psoas muscle and follows the pelvic brim $[1,8]$.

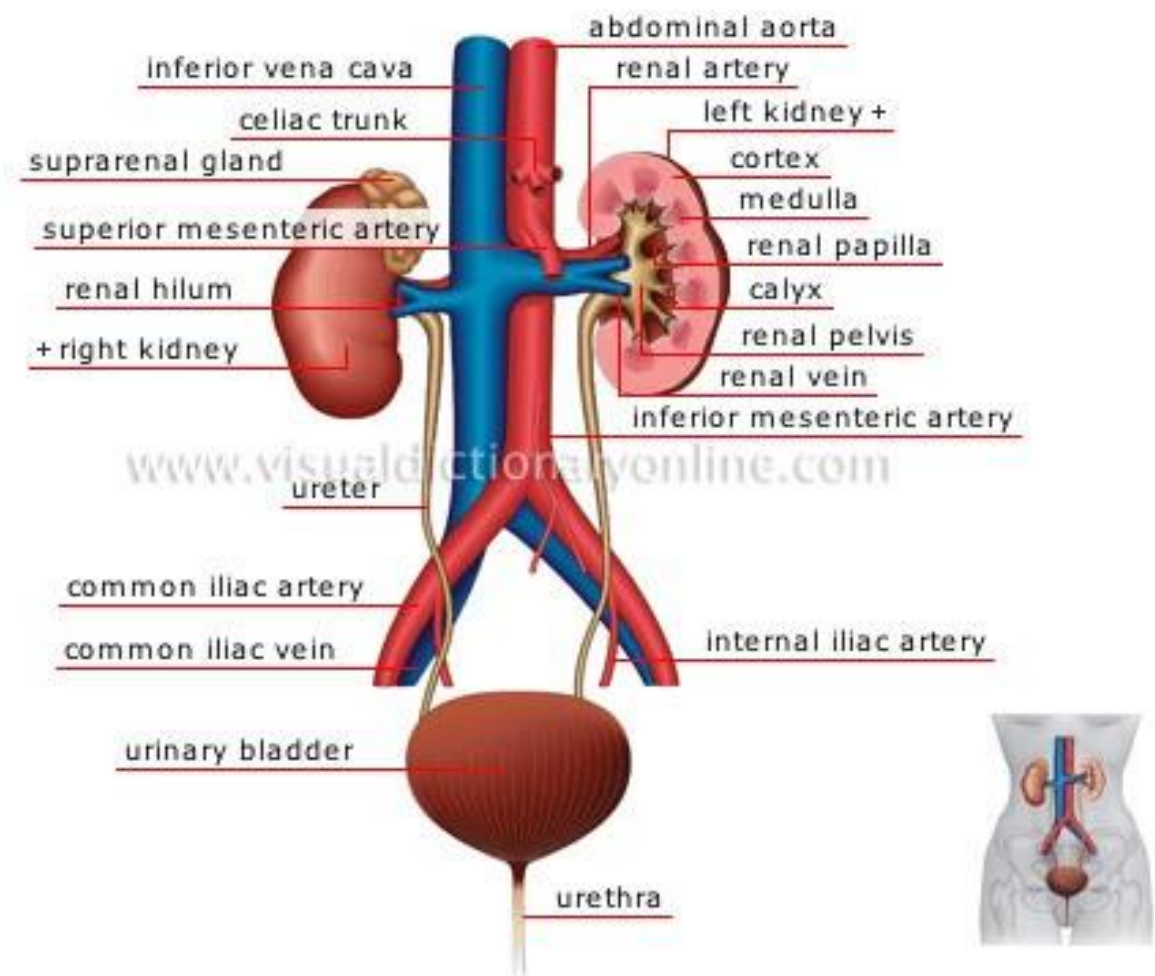

Figure 1: Gross anatomy of the vasculature of interest including the Abdominal Aorta, the common iliac arteries downstream and the internal iliac arteries and external iliac arteries [25].

The average fluid velocity of blood through the abdominal aorta is between 10 and 60 $\mathrm{cm} / \mathrm{s}$, and typical pressures range from $70 \mathrm{mmHg}$ to $115 \mathrm{mmHg}$ [9]. The relatively high pressures in the arterial system necessitate thick vascular walls compared to the venous system which is subjected to pressures of about $20 \mathrm{mmHg}$. A large conducting artery such as the aorta has three major layers to its structure, the tunica intima, tunica media and the tunica adventitia (Figure 2). The tunica intima features the endothelium with the basement membrane underneath the endothelial layer. The endothelium is a selectively 
permeable layer which allows certain substances in and out of the blood stream. The endothelium can also secrete vasodilators and vasoconstrictors, and chemical signals causing leukocytes to adhere to the surface of the endothelium and enter nearby inflamed tissues. Typically the endothelium repels platelets, but in the event that the endothelium is damaged, platelets may adhere to it and form a thrombus. The next major layer is the tunica media, which is composed of smooth muscle, collagen and in the case of large conducting vessels such as the aorta, elastin. The smooth muscle cells in the tunica media are responsible for vasodilation and vasoconstriction which regulate the blood supply received by various tissues. In addition, the tunica media provides a major component of the structural integrity of the blood vessel, preventing rupture under pressure. Finally, the tunica externa consists of connective tissues which secure the vessel in place. It allows passage for small nerves, lymphatic vessels and vasa vasorum which provide oxygen to the outer layers of larger vessels. The interior of most large blood vessels are believed to be oxygenated by blood passing through the lumen $[10,7]$. 


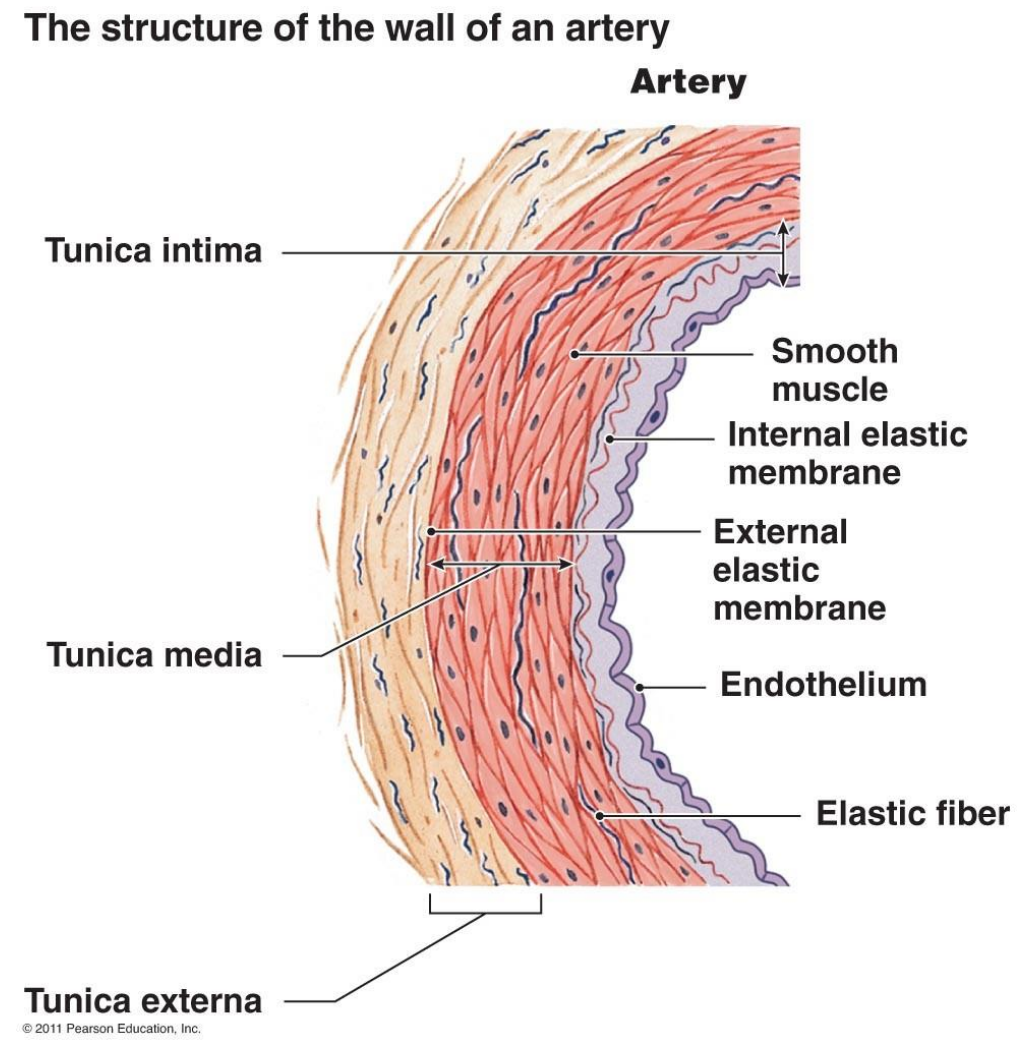

Figure 2: Cross section of a typical artery [26].

\subsection{Etiology and Pathophysiology}

\subsubsection{Risk Factors}

An Iliac Artery Aneurysm (IAA) is often defined as a dilation of the iliac arteries of at least $1.5 \mathrm{~cm}$ while Abdominal Aortic Aneurysms (AAAs) are typically defined by dilations greater than $3 \mathrm{~cm}$ in diameter. Iliac artery aneurysms coincide with about $10 \%$ to $20 \%$ of AAAs. Isolated iliac artery aneurysms are relatively rare, comprising about $0.4 \%$ to $1.9 \%$ of all aneurysmal disease.

Men aged 70 years and older are primarily affected by IAAs, with a sex ratio of 7:1 [1]. Common comorbidities include hypertension, coronary artery disease, peripheral vascular disease and chronic renal insufficiency. Smoking increases the risk of aneurysm 
development [20]. Connective tissue disorders may lead to the degradation of collagen or elastin surrounding blood vessels, leading to dilation and the eventual rupture of the vessel wall [3].

\subsubsection{Mechanisms}

The pathogenesis of IAAs is not well understood, though it is believed to be similar to that of AAAs. Suspected mechanisms include the degradation of elastin on the arterial wall associated with excess matrix metalloproteinase activity due to the presence of macrophages in atherosclerotic plaques, reducing wall strength $[1,11]$.

In the case of AAAs, the most common cause of extracellular matrix degradation is the presence of inflammatory infiltrates and pyrolytic enzymes generated in atherosclerotic arteries. The atherosclerotic plaques inhibit the diffusion of waste products as well as nutrients between the vascular wall and the lumen, leading to ischemia in the media [12]. It should be noted that the importance of atherosclerosis is the subject of dispute. Aneurysms often form in the absence of atherosclerosis, and arteries with heavy atherosclerotic plaque buildup rarely become aneurysmal. It is likely that atherosclerosis may increase the progression of aneurysm growth without being the primary driver of aneurysm formation.

Additionally, hypertension can lead to narrowing of the vasa vasorum, causing ischemia in the outer media. These factors result in necrosis in the media, weakening the arterial wall. Age related degradation of elastin in the tunica media, as well as increased collagen formation causes arteries to become stiffer and less elastic with age. This increased stiffness of the arteries causes the speed of the pulse wave to increase from 
about $6.5 \mathrm{~m} / \mathrm{s}$ at age 10 to about $11 \mathrm{~m} / \mathrm{s}$ at age 60 , contributing to a fatigue-like mechanism where cyclic tearing of elastin fibers combined with gradual loss of elasticity with age leads to a gradual permanent dilation of the blood vessel [41].

Age related changes in the vasculature may also generate hemodynamic conditions that contribute to aneurysm formation. As a person ages, their blood vessels slightly increase in length, leading to increased tortuosity which cause unsteady flow conditions such as increased turbulence which damages the endothelium and causes regulatory processes to malfunction. It has been argued that the responses of endothelial cells to unsteady flow conditions may contribute to the degradation of the vascular wall. However, studies in this field have not yet conclusively proven this connection [41].

Low shear stresses along the endothelium lead to decreased permeability of the vascular wall, and low shear stresses $(<0.4 \mathrm{~Pa})$ contribute to the formation of atherosclerotic plaques that can contribute to aneurysmal growth as described above. Areas where low shear stresses are likely to occur include the lateral surfaces of the lumen at bifurcations, and the intra-aneurysmal lumen. Low shear stress regions exhibit greater endothelial cell cycling and vulnerability to systemic apoptogenic stimuli such as low-density lipoprotein, and tumor necrosis factor (TNF)- $\alpha$. Endothelial cells under low shear stresses may activate circulating monocytes, causing adhesion and diapedesis (monocyte penetration of the vascular wall). Low antioxidant levels in combination with low nitric oxide production stimulate an increased cell cycle in the components of the vascular wall. Specifically, increased production of mitogenic substances like angiotensin-II, endothelin-1, and platelet derived growth factor B increase smooth muscle cell and fibroblast proliferation. Reduced production of fibrinolytic tissue type 
plasminogen activator, nitric oxide, and prostacyclin may increase fibrin deposition and platelet aggregation, therefore accelerating atherosclerotic plaque development, and increase the risk of a thromboembolism occurring [42].

Less common factors contributing to the development of AAAs include mutations in TGF $\beta$ receptors or signaling pathways downstream which lead to the synthesis of defective elastin (e.g. Marfan's Syndrome) [12]. The role of physiological conditions such as wall shear forces or pressure in the degeneration of the arterial wall is currently a subject of debate. It is likely that aneurysmal development is the result of a combination of biological processes such as aging, inherited biochemical or structural defects, and infection as well as hemodynamic or physiological triggers (Figure 1.3) [41].

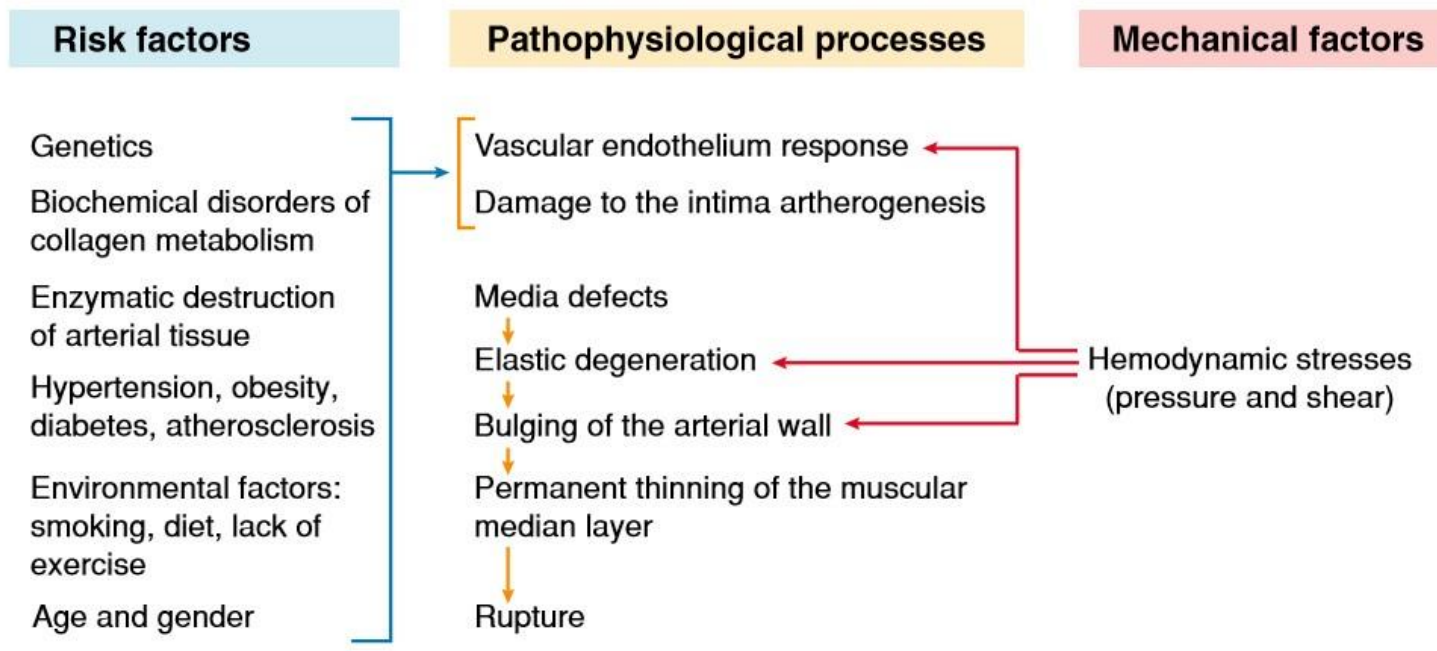

Figure 3: Visual representation of likely interplay of biological and hemodynamic factors contributing to aneurysmal growth [41]. 


\subsection{Natural History}

IAAs are often associated with atherosclerosis, trauma, or infections. Of these causes, atherosclerosis is the most common. Historically, aneurysms were associated with infectious diseases such as syphilis and tuberculosis. One notable subgroup of patients includes young females who underwent pregnancy and suffered from IAAs associated with trauma resulting from Caesarian sections and childbirth [2].

\subsection{Symptoms}

IAAs are symptomatic in up to $50 \%$ of cases as a result of the compression of surrounding tissues. Patients may make complaints about vague lower back, abdominal or flank pain. Other symptoms may include sepsis or pyelonephritis (kidney infection) resulting from urethral obstruction, pain on defecation, and paresthesias (commonly known as a "pins and needles" sensation) of lower legs due to pelvic nerve compression. Diagnosis is often delayed since these symptoms are not a directly related to the cardiovascular system. According to one paper, IAAs can be diagnosed in as many as two thirds of patients by detection of a pulsatile mass during abdominal or rectal interrogation [1].

One case study reported an instance of a 65 year old man with a history of hypertension and smoking who presented with a symptomatic common iliac artery aneurysm. Symptoms included abdominal pain and distension of the abdomen. Clinical examination revealed a pulsatile mass that extended from the right iliac fossa to the paraumbilical region, as well as pulsations in all limbs. CT imaging showed that the patient suffered from an isolated common iliac artery aneurysm. The patient was treated 
by open surgery rather than endovascular repair due to concerns over the tortuosity and calcification present in his blood vessels [15]. Another case study reported a 78 year old man who presented with acute pain in the lower left hip quadrant which was agitated by walking and relieved at rest. In addition, the patient suffered from nausea at the time of his visit, but was otherwise alert. Notable medical history included a previously treated AAA, chronic atrial fibrillation, hypertension, hyperlipidemia, and controlled type II diabetes mellitus. CT scans revealed an 8.5 by $8.0 \mathrm{~cm}$ internal iliac artery aneurysm which likely caused mild to moderate renal hydronephrosis due to compression of the ureter. The formation of a hematoma caused by the accumulation of fluid in the surrounding tissues indicated possible aneurysm rupture. The patient was referred to emergent vascular surgery, but suffered cardiac arrest en route, and was not able to be revived [13].

\subsection{Diagnosis}

Asymptomatic aneurysms are typically discovered during abdominal or pelvic imaging for unrelated conditions. B-mode ultrasonography and computed tomography are typical imaging techniques which can be used to detect aneurysms. B-mode ultrasound is relatively inexpensive and imprecise while computed tomography is more expensive and provides a more precise rendering of aneurysmal geometry. The average size of an isolated IAA at the time of discovery is $5.6 \mathrm{~cm}$, large enough to warrant emergent repair (Table 1) [1]. Rates of early detection are poor, presenting a significant bottleneck on current survival rates. For this reason, the National Health Service in the United Kingdom has begun offering men aged 65 or older ultrasound screenings for AAAs [32]. 
Table 1: Aneurysm Management According to Size

\begin{tabular}{cll}
\hline Aneurysm Type & Diameter & \multicolumn{1}{c}{ Treatment } \\
\hline Abdominal Aortic Aneurysm [17] & Less than $5 \mathrm{~cm}$ & Ultrasound \\
& & Surveillance \\
& Greater than $9 \mathrm{~cm}$ to $8 \mathrm{~cm}$ & Emergent Repair \\
\hline Iliac Artery Aneurysm [1] & Less than $3.5 \mathrm{~cm}$ & Ultrasound \\
& & Surveillance \\
& & Elective repair \\
& Greater than $5 \mathrm{~cm}$ & Emergent repair \\
\hline
\end{tabular}

IAAs are divided into five categories based on the morphology of aneurysmal vasculature. These categories are based on the location of the aneurysm or aneurysms, and the presence of proximal and distal necks at the ends of the aneurysm. The function of these categories is to determine appropriate treatment options. The categories are as follows [1]:

1. Category A: Isolated common iliac artery aneurysm which features proximal and distal necks longer than $1 \mathrm{~cm}$ which features minimal calcification or thrombus formation. Patients may be treated in open repair with aneurysmorraphy using an inlay interposition graft anastomosed proximally and distally to the aneurysm. Endografts must be positioned to attach to the vascular wall at the proximal and distal necks within the common iliac artery. 
2. Category B: Common iliac artery (CIA) aneurysm featuring a proximal neck while the distal aspect of the aneurysm extends into the bifurcation of the CIA. These aneurysms can be treated in open repair using inlay interposition grafts which is anastomosed proximally to the normal CIA, and distally at either side of the bifurcation. In endovascular repair, the graft is laid across the proximal normal CIA and the distal end attached to the external iliac artery (EIA), with the internal iliac artery (IIA) closed off by coil embolization.

3. Category $\mathrm{C}$ : Common iliac artery aneurysm featuring an adequate proximal neck within theca, but where the dilated region extends past the bifurcation to include both the EIA and IIA. Patients with this type of aneurysm can be treated in open aneurysmorraphy with an inlay interposition graft. The graft would need to be anastomosed proximal to the normal CIA, and distally to the EIA, with coil embolization in the IIA. If the IIA is only aneurysmal at the proximal trunk, then it may be treated with a jump bypass to the normal distal IIA. If the distal IAA and its anterior and posterior divisions are affected, then it may be treated by opening the aneurysmal sac and over sewing its branches from within the sac. For endovascular repair, a graft may be fixed to the normal proximal CIA, and fixed distally to the normal distal EIA while the IIA is closed off by coil embolization.

4. Category D: Features an aneurysmal IIA with a normal CIA. Open surgical treatment involves over sewing the IIA branches from within the aneurysm. An inlay graft can be used to bridge the CIA to the EIA if the ostium of the EIA is aneurysmal. For a redundant EIA, then a primary end-to-end CIA-EIA 
anastomosis may be performed. Endografts may also be placed across an aneurysmal IIA as coil embolization is used to close off the aneurysmal sac.

5. Category E: Includes a variety of possible morphologies. Patient may have an isolated CIA aneurysm with inadequate proximal neck due to aneurysmal CIA ostium or aortic bifurcation, or the patient may have a CIA aneurysm which coincides with an AAA, or the patient may have bilateral CIA aneurysms. Patients with a Category E aneurysm may be treated with a bifurcated graft anastomosed proximally just below the renal arteries past the aneurysmal disease to the normal distal sections of the CIAs. For patients where the iliac bifurcation is involved, it open approaches need to employ distal anastomotic techniques such as those used for patients with Category B or C aneurysms. Endovascular techniques may employ coil embolization along with endografts featuring a multi-branched system which extends into the IIA and EIA to repair bilateral CIA aneurysms.

\subsubsection{Ultrasonography}

After examination, ultrasound, or medical ultrasonography is used to verify the presence of an IAA. For ultrasound, images are recorded in B-Mode, which records a two dimensional image of a cross section of the patient's body. Frequencies used to capture these images typically range from 1 to $18 \mathrm{MHz}$. One case reported in the Journal of Diagnostic Medical Sonography described the detection of an internal iliac artery aneurysm by ultrasound performed at $5 \mathrm{MHz}$ using a Philips iu22 device [13]. High frequency pulses are generated by a transducer and sent through the body. Echoes from 
various structures are received by the transducer, which can determine the location of the structure based on the timing of the echo. The brightness of a spot on a sonogram is determined by the intensity of an echo, with more intense echoes producing brighter images on the display. A sweeping action is required to determine the location of an echo source. This can be accomplished with a one dimensional array of transducers which perform the sweep electronically [5].

Ultrasound is the most commonly used method of screening for AAAs in the U.S. There is no universally accepted practice for detection, but the three dominant imaging techniques are inner-to-inner edge (ITI), leading-to-leading edge (LTL), and outer-to outer edge (OTO). A study comparing the three methods found no statistically significant difference between the three methods in terms of inter and intra observer variability. However, since the ITI method measures the size of an aneurysm from the inner aortic walls, it does not account for the combined $4 \mathrm{~mm}$ thickness of the aortic walls, resulting in an underestimation of the aneurysm size. This discrepancy between the output of ITI and OTO methods needs to be accounted for by adjusting surveillance and treatment thresholds to the imaging method [14].

\subsubsection{Computed Tomography}

Computed tomography is a method of imaging internal structures non-invasively. Like ultrasound, CT scans generate 2D images. However, CT scans have the advantage of producing higher resolution images. Due to their high cost, CT scans are not generally used to verify the presence of an aneurysm, but to determine the dimensions of the aneurysm and surrounding blood vessels prior to surgery. 
$\mathrm{X}$-rays are generated by heating a tungsten filament enough to emit electrons, which collide with a metal plate (such as copper) and emit x-rays during deceleration. These $\mathrm{x}$ rays pass through the patient's body and hit a detector. The detector records the attenuation of each ray in the fan-shaped x-ray beam in each view of the body. The data generated by multiple views can be used to reconstruct an image of the internal structure of an axial cross section of the patient. In hospitals, the beam is swept around the patient in a spiral pattern, recording overlapping cross sections of the body. This generates sufficient volumetric data to allow the reconstruction of three dimensional models of the internal structures of the patient's body. In CT angiograms, a contrast agent is injected into the patient's blood to increase the contrast of the vasculature. Typically iodine based solutions are used, since they have few associated side effects. CT angiography allows high resolution, high contrast images of the vasculature of a patient, allowing for detailed models of structures such as aneurysms to be reconstructed [6].

Another technique for the imaging of aneurysms is multi-detector computed tomography (MDCT) which is reportedly replacing conventional angiography in many instances. Unlike conventional CT scans, which use a one dimensional array of detectors that necessitate a helical sweep, multi-detector CT scans employ a two dimensional array of detectors and is able to obtain multiple slices simultaneously, reducing the time required for image acquisition. MDCT has the ability to assess peri-aortic soft tissue and resolve aneurysmal dimensions with high precision. Its excellent vascular opacification and multi-planar reconstruction of vasculature make MDCT a good technique for imaging abdominal aortic and iliac artery aneurysms [16]. 


\subsection{Treatments}

Historically, iliac artery and abdominal aortic aneurysms were repaired by open surgery, where the aneurysmal segments of blood vessel were replaced with vascular grafts. However, open surgery is associated higher morbidity and mortality rates than minimally invasive Endovascular Repair (EVAR) procedures. Limitations of endovascular grafts include graft migration from the implant site and endoleaks, which can result in aneurysmal sac expansion. EVAR graft patients require reintervention in $15 \%$ to $19 \%$ of cases after an average of 34 months [18].

\subsubsection{Endovascular Repair}

Endovascular repair involves the transcatheter implantation of a stent graft in the lumen of a blood vessel at the site of an aneurysm. When properly secured, the graft reinforces the blood vessel and prevents blood from leaking into the aneurysmal sac. This halts aneurysmal growth and prevents rupture. The precise configuration of the stent graft depends on the location of the aneurysm and vascular geometry. For example, in the Chemelli study, iliac artery aneurysms with necks at least $1.5 \mathrm{~cm}$ long were considered sufficient for the placement of a stent graft. For aneurysms that did not feature adequate necks of healthy vasculature, a bifurcated stent graft or an abdominomonoiliac stent graft was used. Aneurysms with insufficiently long distal landing zones were treated with grafts that extended into the external iliac artery. Moreover, the dimensions of the stent grafts were determined with the aid of preinterventional CT scans [19].

Scheinert et al. [21] reported a primary technical success rate of 97.9\% (47 of 48 cases) with one case of residual perfusion of the aneurysmal sac, which resolved itself 
spontaneously within several days of surgery. 62.2\% (23 of 37) of patients with claudication or resting pain reported improvements at the two week follow up. Minor complications including a groin hematoma, one false aneurysm, eight patients treated with the EndoPro System 1 elevated body temperature (mean = $38.8^{\circ} \mathrm{C}$ ), and 7 patients suffered from increased white blood cell counts (mean $=$ 15.6/nL). Kaplan Mayer estimates indicated a postoperative patency rate of $100 \%$ for the first 12 months, which dropped to $87.6 \%$ at the 48 month mark. There are no statistically significant associations between method of repair, gender, or comorbidities (e.g. hypertension, diabetes, coronary artery disease, or hyperlipidemia) and death. Higher iliac artery morphologic scores (assigned according to Society for Vascular Surgery guidelines) and higher rates of bilateral hypogastric occlusion were found to be associated with death with $95 \%$ confidence [20].

\subsubsection{Open Repair}

In open repair, the diseased portion of the patient's aorta is cross clamped and cut open to allow a polymeric graft, often made from Goretex (PTFE), to be sutured into place. The aneurysmal sac is then sutured around the graft. Historically, open surgery was the standard method of repairing AAAs and IAAs. However the procedure has fallen out of favor in the past decade with the advent of less invasive endovascular repair (EVAR) procedures. Nevertheless, open repair is still considered an option for patients who are younger and better able to withstand the physiological stress of surgery, and for whom long term durability of the graft is a greater concern. Conrad et al. [23] reported that open repair of AAAs are associated with a $98.2 \%$ freedom from reintervention rate compared 
to a $78.2 \%$ freedom from reintervention rate for patients who underwent EVAR. Moreover, it was reported that while EVAR is associated with lower perioperative mortality rates compared to Open Repair, patients who undergo open repair exhibit greater long term survival rates. Conrad et al reported 5 year survival rates of $70.7 \%$ for open repair compared to $52.1 \%$ for EVAR. On the other hand, Duran et al. [20] reported $100 \%$ morbidity post-operatively (within 30 days of surgery) for open repair of iliac arteries compared to $23 \%$ for endovascular repair of iliac arteries. Lower short term morbidity and mortality rates account in part for the popularity of endovascular aneurysmal repair, and suggest that it may be the appropriate option for patients who are older and higher risk for open repair.

\subsection{Previous Research into Aneurysmal Hemodynamics}

A study by Castro et al. [35] sought to determine the effects of upstream vascular geometry on aneurysmal flow fields. Patient angiograms were used to generate meshes for FEA simulations. The flow rates used in these simulations were obtained from NMR measurements on different patients on the same arteries. Simulations were run for models containing the original upstream vasculature, and for models which were modified such that the parent arteries were truncated $1 \mathrm{~cm}$ upstream from the aneurysm, and replaced with straight cylinders. In all cases, the simulations featuring the truncated parent vessel underestimated wall shear stress within the aneurysm, and shifted the impaction zone towards the neck. Additionally, the laminar behavior of flow in the parent vessel caused the aneurysms to exhibit less complex flow fields. 
The sensitivity of model behavior to geometry indicates that in vitro models with idealized geometries are of limited clinical value in understanding the hemodynamics presented in individual cases, because, predictions of physiological responses to hemodynamic conditions seen in in vitro models can be unreliable. In response, research in to aneurysmal hemodynamics has shifted away from the use of idealized models to realistic models from multiple patients, allowing connections to be drawn between patient specific hemodynamic factors and clinical events [35]. For example, Cebral et al. [33] acquired dimensional information about intracranial aneurysms in 80 patients using CT angiography. Then simulations of blood flow under pulsatile conditions were conducted. Assumptions included Newtonian behavior, rigid walls, no-slip boundary condition at the vascular wall, and laminar flow. In this study, a total of 62 fluid flow models were produced across 57 patients, and the study drew associations between subarachnoid hemorrhage risk and intra aneurysmal fluid flow behavior. It was found that ruptured aneurysms exhibited complex and unstable flow patterns, small impingement regions, and narrow inflow jets. By contrast, aneurysms with simple flow patterns, small impingement regions, and large jet sizes. The hemodynamic conditions in an aneurysm were highly dependent on patient specific aneurysmal geometry.

Existing imaging methods can provide rough estimates of hemodynamic conditions in vivo. For example, CT imaging synchronized with the cardiac cycle can determine fluid velocity based on contrast dye wash in and wash out times. NMR may be used to characterize velocity profiles with the phase contrast technique, because the measured phase of a medium is dependent on its velocity. Ultrasonography and echocardiography may be used to characterize the in-plane component of velocity profiles [34]. However, 
no existing non-invasive or invasive techniques are able to provide a detailed characterization of velocity fields, which would be needed to completely validate the computer simulations. This issue can be circumvented by constructing in vitro models of patient aneurysms, recorded by angiograms, for empirical validation of computer simulations.

Tateshima et al. [36] created an in vitro model of an intracranial aneurysm to allow empirical validation of computer simulations of intra-aneurysmal fluid flow fields. The aneurysm model used in the study was extracted from an angiogram, and used to create a silicone construct. An aqueous solution of glycerol was chosen to match the refraction coefficients of the fluid and the mold and minimize optical distortion in the resulting images. Additionally the aneurysm model was scaled up $3 x$ to allow detailed observation of the flow fields. Individual parameters used in the study were modulated to ensure dimensionless parameters such as the Reynolds number and Wormersly pulsatility index matched values seen in vivo. Velocity and vorticity measurements were recorded by PIV analysis. Wall shear stress measurements were calculated based on velocity data. High shear stress levels at the neck of the aneurysm were observed, corroborating evidence that shear stress conditions at the neck stimulates matrix metalloproteinase activity, leading to degradation of the arterial wall from the neck of the aneurysm [36]. Other research has indicated that shear stresses below $0.4 \mathrm{~Pa}$ are associated with the development of atherosclerotic plaques at the lateral edges of aneurysmal bifurcations [42].

Due to the unusual nature of iliac artery aneurysms, no research was found discussing in vitro models of IAAs. However, previous researchers have conducted in vitro studies 
of AAAs using silicone models. For example, Ene et al. [40] obtained angiograms and generated an idealized 3D model of the AAA based best-fit circles fitted to a straight centerline. The best fit circles were swept to generate the surfaces of the model. This idealized model of the aneurysm was used these to produce models of the vasculature to be used in the study. Three molds were made, which allowed the manufacture of the aneurysm for this study.

1. Mold A was used to produce the wax "core" used to define lumen geometry.

2. Mold B created the intra-lumen thrombus

3. Mold C defined the exterior wall of the aneurysm

Pulsatile flow based on a physiologically realistic waveform was introduced into the model, and the extension and distension of the wall was recorded by a camera to characterize the mechanical behavior of aneurysmal vasculature. It was found that the intra-lumen thrombus reduced arterial compliance by between $0.5 \%$ and $81 \%$. The proximal region of the aneurysm exhibited greater compliance, and presented with the greatest strain, making it a likely site for rupture [40].

\subsection{Fluids Theory}

\subsubsection{Flow Through Closed Channels}

The fluid flow model assumes a solid conduit (e.g. a cylindrical conduit), meaning there is a no-slip condition at the conduit surface. Volumetric flow driven by a pressure differential across the conduit can be modeled using Hagen-Poiseuille. Boundary conditions include no-slip at the walls, and a shear rate of zero at the center, where the fluid is at maximum velocity (Equation 1.1). 


$$
Q=\frac{\pi R^{4}\left(P_{i}-P_{o}\right)}{8 \mu L}
$$

Where $\mathrm{Q}$ is the volumetric flow rate, $\mathrm{R}$ is the radius of the vessel, $\mu$ is the dynamic viscosity, $P_{i}$ is the pressure at the inlet and $P_{o}$ is the pressure at the outlet, and $\mathrm{L}$ is the total length of the vessel between the inlet and outlet [38].

Fluid flow through a closed channel can be modeled under three different regimes: Stokes (creep), laminar, or inviscid (turbulent) flow. Each of these models describes fluid flow based on the relative roles of viscous and inertial forces in velocity profiles and fluid behavior. The ratio of inertial forces to viscous forces can be described by the Reynolds Number (Equation 1.2).

$$
R e=\frac{\rho v D}{\mu}
$$

Where $\rho$ is the fluid density; $\mathrm{v}$ is the magnitude of the fluid velocity; D is the hydraulic diameter; and $\mu$ is the dynamic viscosity [38].

The following table (Table 2) describes the relationship between flow regimes and Reynolds Number. The dimensionless form of the Navier-Stokes equation may be used to describe flow conditions exhibiting in a closed vessel (Equation 1.3):

$$
\operatorname{Re}\left(\frac{\partial \hat{v}}{\partial \hat{t}}+\hat{v} \cdot \hat{\nabla} \hat{v}\right)=\hat{\nabla}^{2} \hat{v}-\hat{\nabla} \hat{\mathbb{P}}
$$

Where $\hat{v}$ is the velocity vector, Re is the Reynolds Number, and $\widehat{\bigotimes}$ represents modified pressure. 
Table 2: Flow Regime According To Reynolds Number

\begin{tabular}{ccl}
\hline Flow Type & Reynolds Number Range & Dominant Forces \\
\hline Stokes Flow & $R e<<1$ & Viscous Forces Dominate [37] \\
Laminar Flow & $1<R e<2100$ & Viscous and Inertial Forces Contribute \\
Transient Flow & $2100<R e<4000$ & Increasing Inertial Contribution [38] \\
Inviscid Flow & $R e>4000$ & Inertial Forces Dominate [38] \\
\hline
\end{tabular}

For Reynolds Number values close to zero, viscous forces dominate, and fluid flow can be described without accounting for inertial effects. The Stokes flow model is identical to the laminar model, except the inertial term is assumed to be zero, and is therefore dropped from the Navier-Stokes equation (Equation 1.4). [46] In a physiological context, this equation is valid for describing flow in capillaries, arterioles, and venules. It may be used to describe flow through microfluidic devices as well.

$$
\rho \frac{d \vec{v}}{d t}=\mu \nabla^{2} \vec{v}-\nabla P+\rho \vec{g}
$$

Where $\rho$ is the density of the fluid, $\mu$ is the (constant) dynamic viscosity, $\sim v$ is the velocity of the fluid, $\mathrm{P}$ describes the pressure, and $\sim g$ gives the gravitational acceleration that contributes to a body force. In a physiological context, pulsatile flow would be described by representing $\frac{d \vec{v}}{d t}$ as a sinusoidal function. For constant flow rates, $\frac{d \vec{v}}{d t}=0$ and the equation simplifies to the following (Equation 1.5):

$$
0=\mu \nabla^{2} \vec{v}-\nabla P+\rho \vec{g}
$$


Under laminar conditions or transient conditions, both the inertial effects and the viscous effects need to be taken into account for accurate predictions of flow (Equation 1.6). Physiologically, flow in the arteries and veins can be described using a model that accounts for the inertial terms [47].

$$
\rho\left(\frac{\partial \vec{v}}{\partial t}+\vec{v} \cdot \nabla \vec{v}\right)=\mu \nabla^{2} \vec{v}-\nabla P+\rho \vec{g}
$$

In the context of this project, the Reynolds Number in the abdominal aorta in the half vessel model is approximately 3200, a value where transient behavior could be expected. The COMSOL simulation used in this project treats the flow as laminar so that both inertial and viscous effects were accounted for in the model.

\subsubsection{Fluid Properties}

Most fluids, including water, are Newtonian fluids that exhibit a shear rate that varies linearly with shear stress (Equation 1.7). Therefore, the viscosity, $\mu$ is a constant. The in vitro studies used in this experiment employed water, with a viscosity of $1.0 \mathrm{cP}$, and therefore this property was transmitted to COMSOL to validate the experiment. Blood is often modeled as a Newtonian fluid, with a viscosity of about $3.0 \mathrm{cP}$ to $4 \mathrm{cP}$. This assumption can be useful at relatively high shear rates [38, 35].

$$
\tau_{r z}=-\mu \frac{d v_{x}}{d y}=\mu \dot{\gamma}_{r z}
$$

Where $\tau_{r z}$ is the shear stress at the inner surface of the vessel, $\mu$ is the dynamic

viscosity, and $\frac{d v_{x}}{d y}$ describes the shear rate $\left(\dot{\gamma}_{r z}\right)$ which is obtained by describing the change in velocity of the fluid in the $\mathrm{x}$-direction with respect to changes in position in the y-direction [38]. 
However, since blood contains various cells and proteins, its behavior is more complex than a Newtonian fluid. Non-Newtonian fluids exhibit a non-linear relationship between shear rate and shear stress, meaning $\mu$ cannot be described as a constant for such fluids. Heterogeneous fluids which feature a particulate phase that aggregates at low shear rates exhibit a shear stress. In the case of blood, red blood cells act like a particulate and aggregate to form roulleaux - structures what are analogous to a stack of coins. At low shear rates, the apparent viscosity of blood is large due to the presence of roulleaux. As the shear rate increases, the apparent viscosity of blood decreases and approaches an asymptote at about $3.0 \mathrm{cP}$. Therefore, blood can be modeled as a Casson fluid. For shear rates above $150 \mathrm{sec}^{-1}$, blood can be treated as a Newtonian fluid with a viscosity of 3.0 $\mathrm{cP}$, since the roulleaux are fully dissociated into individual red blood cells (Figure 4) [38]. 


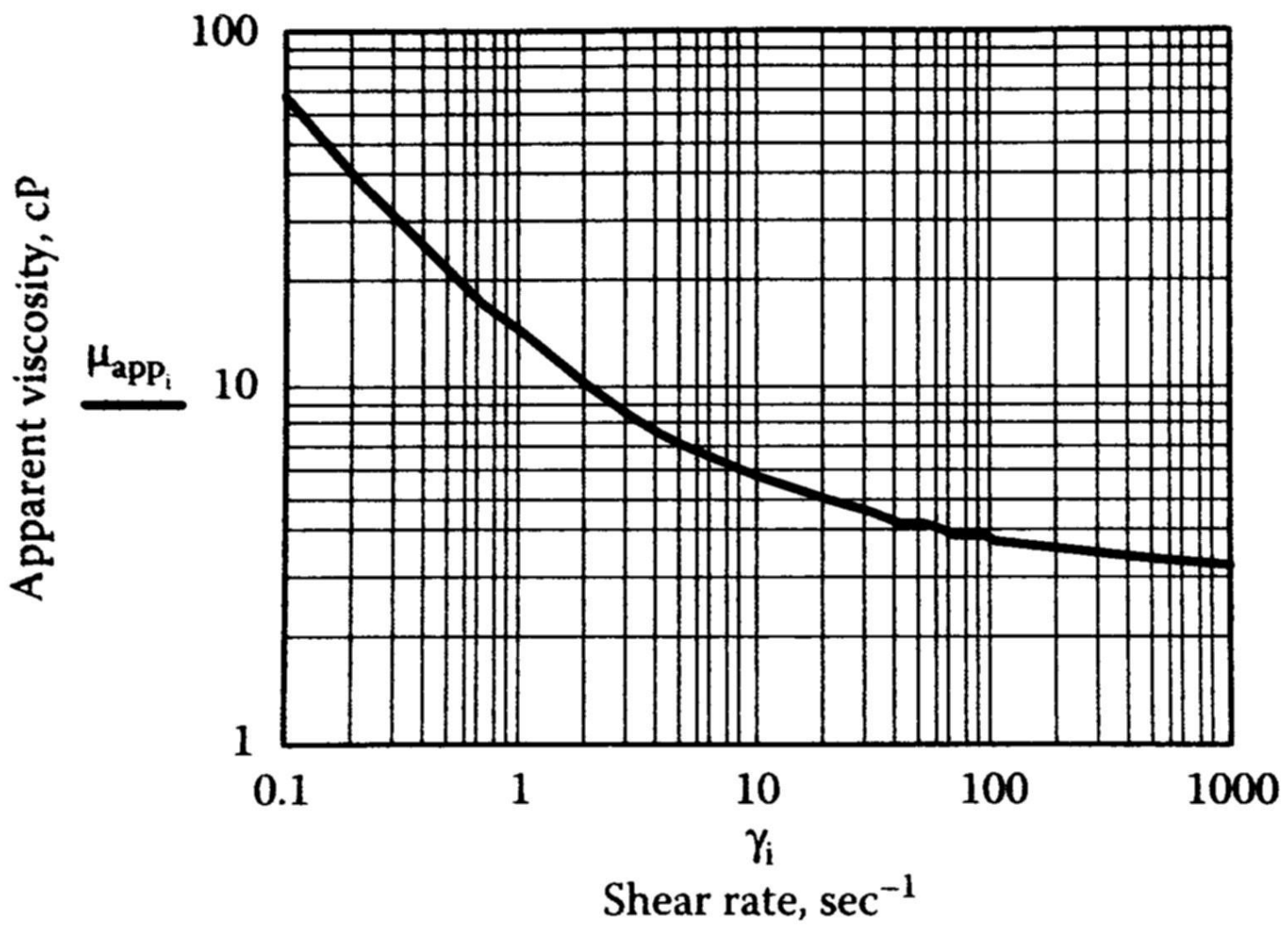

Figure 4: As the shear rate increases, the apparent viscosity of blood decreases and approaches an asymptote at $3.0 \mathrm{cP}$. Therefore, blood is considered to be a shear thinning fluid which can be treated as Newtonian at shear rates higher than $150 \mathrm{sec}^{-1}[38]$. 


\section{MATERIALS AND METHODS}

\subsection{Background}

\subsubsection{Fused Deposition Modeling}

Fused Deposition Modeling (FDM), commonly referred to as 3D printing, is a manufacturing process which offers the ability to produce customized parts without need for retooling, proving useful in research and product development where design considerations prioritize easy customization for low volume production runs. The Aneurysm model used in this project was designed in SolidWorks based on typical dimensional parameters of common iliac artery aneurysms. The STL file produced in CAD was processed in a slicer program (CatalystEX for the uPrint, or KISSlicer for the Series 1), which converts the model into instructions for the printer (known as gcode). Next the model was manufactured by fused deposition modeling (FDM), an additive manufacturing process where a thermoplastic (typically ABS or PLA) material is heated and extruded from the hot end, which repeatedly draws outlines of the cross section of the workpiece in the $\mathrm{x}-\mathrm{y}$ plane. Between layers, the hot end moves up along the $\mathrm{z}$-axis in programmed steps to produce the part layer-by-layer (Figure 5) [28]. Limitations of FDM include build surface size $(203 \times 152 \times 152$ mm for the uPrint, or 228.6 × 228.6 × 228.6

$\mathrm{mm}$ for the Series 1), need for support material where the model includes overhangs, and ridge-like structures coinciding with individual layers, which create a need for post processing when a smooth surface is desired. In cases where STL models include overhangs, adding support material becomes necessary to prevent warping. Some FDM machines use a dual extrusion system which allows it to use a water soluble polymer such as polyvinyl alcohol (PVA) to print the support material for the part. Printers with a 
single extruder require the slicer program to generate a scaffold in the machine instructions which is made of the same material as the part. This support material can be peeled away manually after printing. The latter method of generating support material may result in increased surface roughness where the support material was attached to the part. Ridges generated during the print by the separate layers can be modulated by adjusting the layer height of the print, with smaller ridges for shorter layer heights. Post processing techniques such as polishing or sanding may be used to remove these ridges manually.

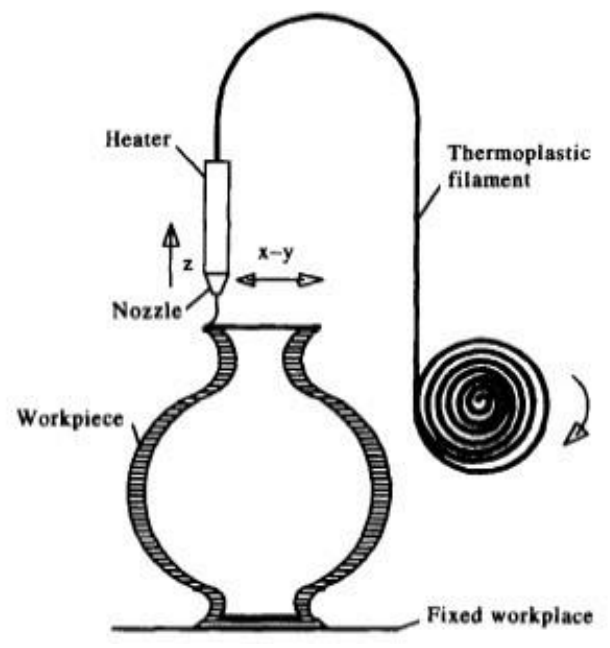

Figure 5: Schematic of FDM operation. A 3D model is split into 2D cross sections which are added drawn by an extruder layer-by-layer to produce a 3D object. Typically the layer height is about $200 \mu \mathrm{m}$ [28].

\subsubsection{Model Preparation}

Before manufacturing by FDM, models produced in CAD programs such as SolidWorks must be converted into STL format, and then into machine instructions. The model output by 3D modeling software is approximated by interconnected triangles (or facets) fit to the CAD model in a 3D Cartesian coordinate system. The STL file format 
consists in a list of facets. Information about each facet is uniquely identifiable by the unit normal, a vector with a length of 1.00 that is perpendicular to the facet surface, and three vertices. Each facet is described by 12 numbers, 3 for the unit normal, and 3 for each of the vertices of the triangle. To differentiate the inside of the model from the outside, the unit normal must point outward, and the vertices are listed in counterclockwise order when viewed from the outside. This convention is known as the "right hand rule" (Figure 6) [27].

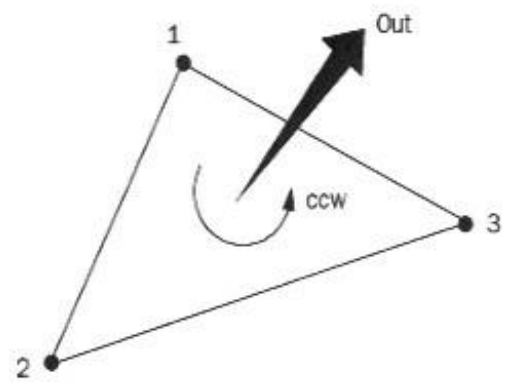

Figure 6: Orientation of individual facet [27].

The vertex-to-vertex rule requires each triangle to share two vertices with each adjacent triangle, such that two triangular facets cannot share a side with the same facet (Figure 7). Facets in an STL are located in the all positive octant of the 3D Cartesian coordinate system so that no negative numbers are used. Special building parameters may be used, such as a minimum triangle side, or a maximum triangle size. Numerical data describing the unit normal and facet vertices are stored in the form of single precision floating point numbers. Finally, it should be noted that units used in an STL are arbitrary, and so scaling may be required when the model is loaded into a slicer in 
preparation for printing, since units specified in the CAD program are lost when the model is exported to an STL file [27].
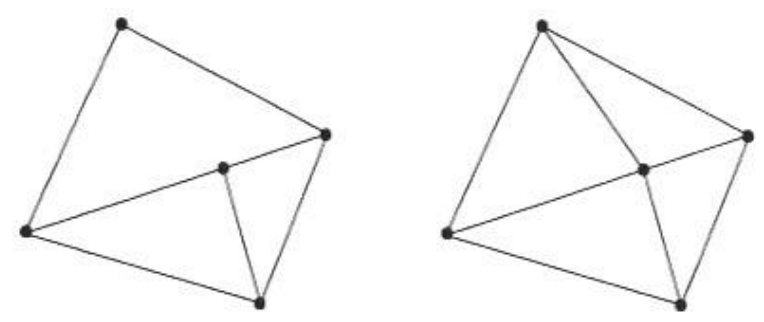

Figure 7: Vertex-to-vertex rule requires vertex coordinates to be shared between facets, prevents vertices from meeting side of facet [27].

The STL file output from CAD is used by computer aided manufacturing (CAM) software, also known as slicer software, to generate instructions (gcode) for the FDM machine. Instructions specify the movements of the print head in the $x-y$ plane, the movement of the build surface in the z-axis, when the hot end should extrude material and the rate of extrusion. Slicers may allow the user to define wall thickness at the surface of the model, layer height, fill patterns which allow material to be saved when a solid part is not desired, print head speed, and support material parameters such as a critical angle of overhang at which to include a scaffold, or the inclusion of a raised bed or raft for the part. Scaffold geometry, fill pattern geometry, and rafts are generated automatically by the slicer based on the chosen parameters and STL model geometry. Slicers can also generate estimates for the amount of material needed, and material cost of a print. Additionally, many slicers provide a preview of the generated machine instructions in the form of a three dimensional model of the route taken by the hot end, 
and can be inspected visually by the user before printing. Finally, the gcode is read by the software client for the FDM machine as the part is manufactured.

\subsubsection{Molding}

An FDM manufactured aneurysm construct was used to create a polydimethylsiloxane (PDMS) vascular half-construct and a full vessel construct of an isolated iliac artery aneurysm. For each of the two constructs, 2.0 kilograms of PDMS from the SYLGARD 184 SILICONE ELASTOMER KIT was used. For the half vessel construct, PDMS was poured around the vessel model so that the mold could be mounted to a glass slide and have dyed water run through it for fluid flow analysis. This process was analogous to the soft lithography process used in microfluidics performed on a larger length scale. The full vessel construct would be created by printing the full aneurysm model using PVA, which could be immersed in PDMS to create the full vessel mold and washed out after curing, to leave a void in the shape of the aneurysm model in a PDMS block. Fluid flow through the full vessel construct may be characterized by dye tracking as well, although there would have been a need to correct for optical distortion due to a refractive index differential between the PDMS and the fluid.

PDMS is a silicon based elastomer which is commonly used in medical devices because it is biocompatible and in microfluidics due to its ability to easily conform to sub-nanometer scale structures through the soft lithography process [43]. This ability of PDMS to conform to small structures means the mold should closely replicate the geometry of the rapid prototyped model, and that artifacts of the FDM process such as ridges marking individual layers will carry over to the PDMS mold. Therefore, post 
processing of either the PDMS or the rapid prototyped vessel model would be necessary to minimize artifacts from the print that could disrupt fluid flow and increase discrepancies between empirical observations and COMSOL model predictions. After curing, PDMS may be temporarily sealed to flat surfaces, such as glass by exploiting van der Walls interactions, or it may be permanently sealed after a plasma treatment to allow stronger polar intermolecular bonds to form between the PDMS and glass [29]. Exposure to plasma creates - $\mathrm{OH}$ groups on the surface of the PDMS, which are converted into $\mathrm{Si}$ O-Si bonds when the PDMS surface is brought into contact with plasma treated glass [43]. PDMS can be cured using a platinum catalyst agent at room temperature, allowing easy processing as heat treatments are not required.

\subsection{CAD Modeling}

The three dimensional model of the aneurysm and surrounding vasculature was created in SolidWorks. The diameters of each vessel were based approximately on the dimensions in given the available literature. The aorta was given a $20 \mathrm{~mm}$ diameter, the common iliac arteries were taken to be $10 \mathrm{~mm}$ in diameter, and the internal and external iliac arteries were assumed to be $8 \mathrm{~mm}$ in diameter based on the ratio of the diameter of the internal and external iliac arteries to that of the common iliac arteries based on measurements of an angiogram, which was not saved at the time [7]. For comparison, a new angiogram was analyzed in ImageJ by taking three measurements across each blood vessel with the straight line tool, and the dimensions were scaled according to the assumption that the aortic lumen was $20 \mathrm{~mm}$ in diameter (Figure 8). The average of each set of three measurements was taken to represent the diameter of the artery (Appendix 
B). Diameters were found in this manner for the CIA $\left(D_{C I A}=13.69 \mathrm{~mm}\right)$, IIA $\left(D_{I I A}=\right.$ $9.04 \mathrm{~mm})$ and EIA $\left(D_{E I A}=9.27 \mathrm{~mm}\right)$ [44]. Vascular geometry is highly variable across patients; Armon et al. reported a 32\% variance within two standard deviations about the reported mean CIA diameter. Therefore, it is reasonable to assume that the dimensions upon which aneurysmal model used in this study are based would fall within a range that might be expected among patients. Furthermore, due to variability in vascular geometry and hemodynamic conditions, conclusions about flow derived from one model of an isolated CIA aneurysm are particular to that geometry and cannot be generalized across patients suffering from isolated CIA aneurysms [8]. In SolidWorks, a straight line was drawn for the aorta. Two splines were branched off the aorta, which made up the common iliac arteries and the external iliac arteries. 


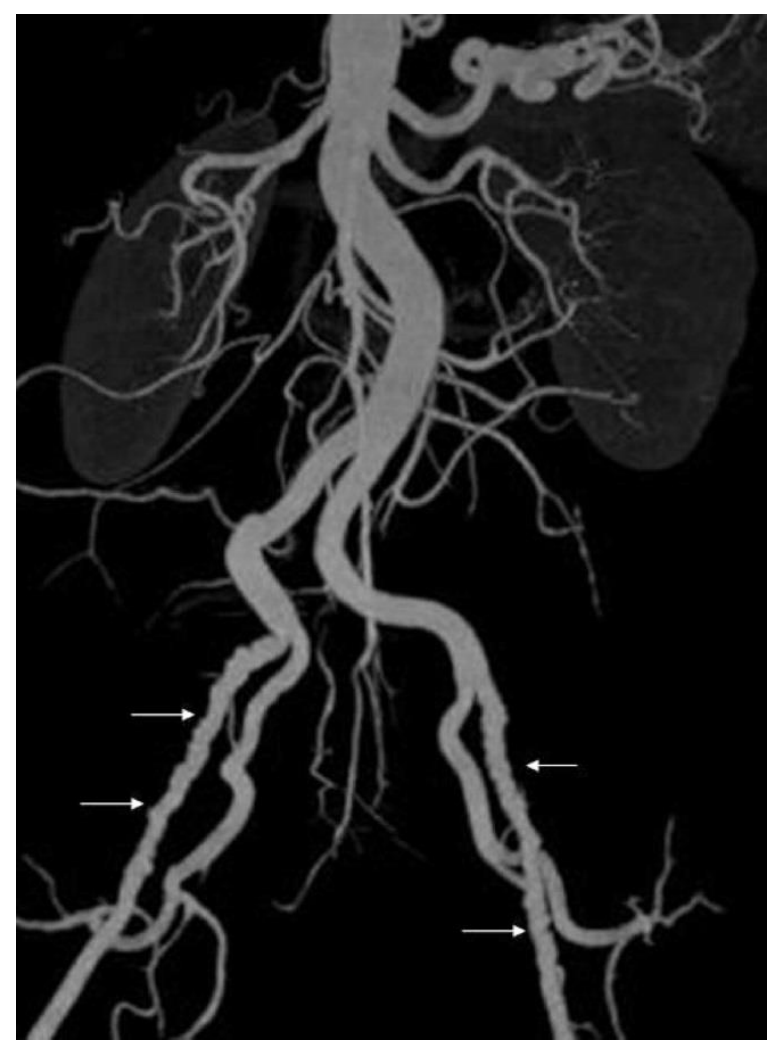

Figure 8: Abdominal angiogram of a patient suffering fibromuscular dysplasia, a condition unrelated to aneurysmal disease. This condition resulted in beading shown on the external iliac arteries, but did not affect the overall arterial dimensions [44].

Near the mid-point of each spline, an additional spline was added to create the internal iliac artery. The vessels downstream of the aorta were assigned curvature arbitrarily. Next, points were drawn at roughly regular intervals along the aorta and all iliac arteries. At each point, an orthogonal plane was added, and then circles centered at each point to be drawn along the new planes. A loft was used to connect each circle to form the blood vessels. Finally, fillets were added to smooth out sharp edges at the branches of the model (Appendix C). In COMSOL, the model was split in half longitudinally so there would be a flat surface to face the glass slide during imaging. In preparation for FDM 
manufacturing on a limited build surface, the model was split into four pieces; the aorta, common iliac arteries and aneurysm, two sets of internal and external iliac arteries. Rectangular pegs and holes were added to each piece near the flat surface to allow them to fit together when assembled (Figure 9). Finally, a $3 \mathrm{~cm}$ long half-cone attachment was added to the aorta with a $3 / 16$ inch peg to allow tubing to attach to the mold later (Figure $10)$.

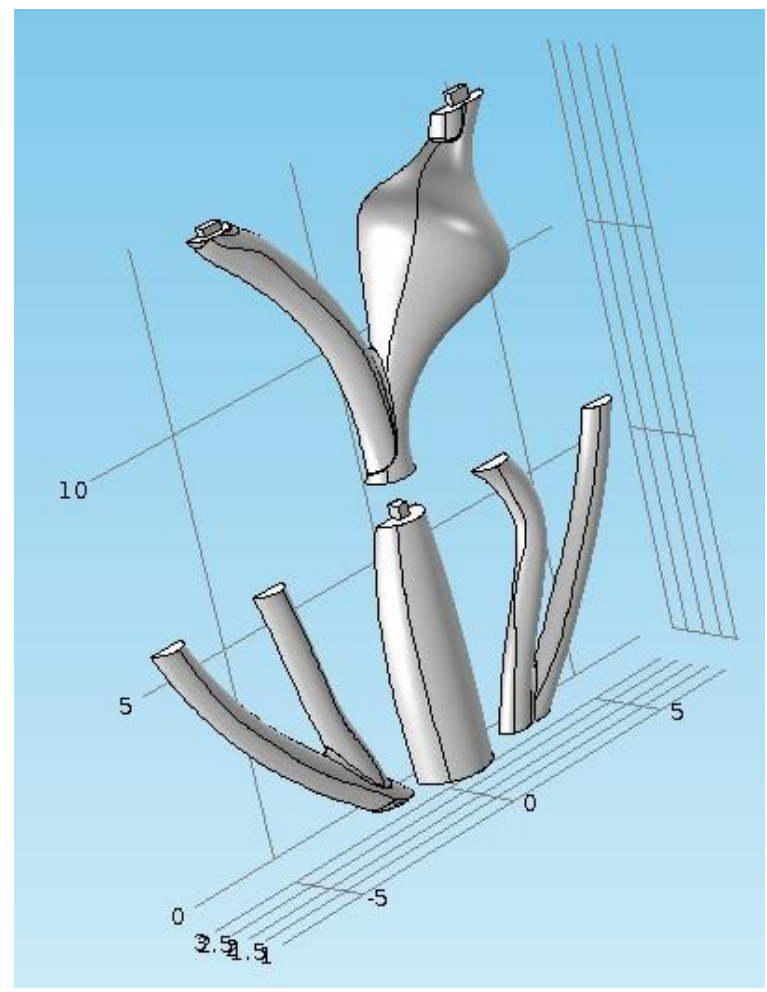

Figure 9: To ensure the half-vessel model could be printed, the model was split into multiple pieces with pegs and holes added, and arranged to ensure all pieces would fit the uPrint build surface. At this point, the conical connector had not been added, which is why the aortic section was modified and reprinted in PLA on the Series 1 at a later date. 


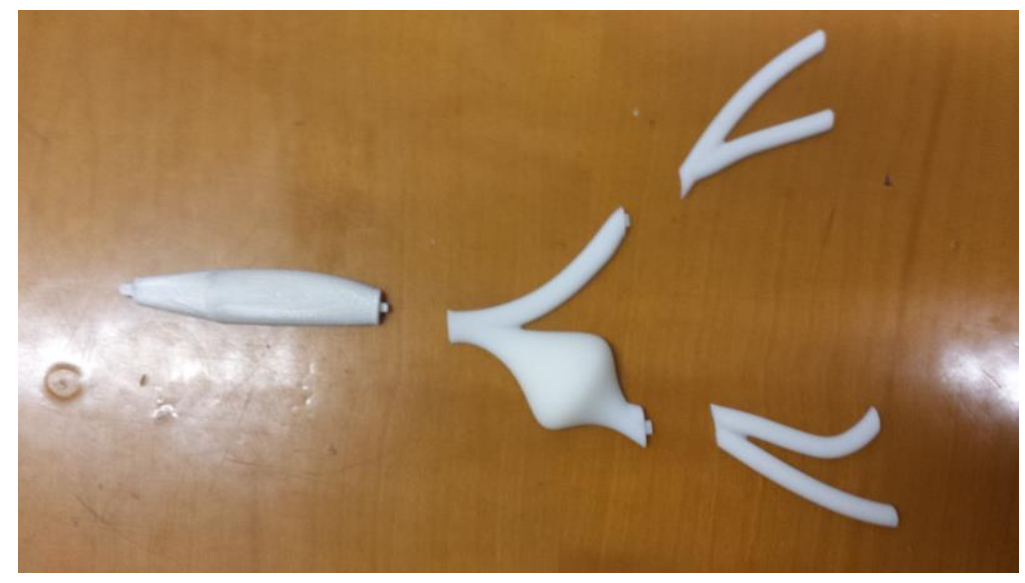

Figure 10: Aneurysm model after FDM manufacturing. The aneurysm model was assembled and used in the molding process to define the shape of the channel of the in vitro model. The aortic section was modified to add the conical connector with a 3/16 inch diameter peg.

\subsection{Half Vessel Construct}

The half vessel construct was comprised of a mold of the vascular model split in half longitudinally along the $\mathrm{x}-\mathrm{z}$ plane seen in COMSOL, with the flat face resting on a glass slide. The glass slide functioned as a window into the model for later imaging trials and prevented optical distortion by virtue of its flat geometry.

\subsubsection{FDM Manufacturing}

The majority of the model was manufactured on a uPrint machine using ABS with $100 \%$ infill and a $200 \mu \mathrm{m}$ layer height. With the exception of a water soluble raft at the bottom of each component, no support material was used. Because the attachment for the tubing was added to the aortic model later on, the aortic section of the model was manufactured on a 2012-2013 Type A Machines Series 1 using PLA, with $16 \%$ infill and a $200 \mu m$ layer height without any support material. Additionally, the Series 1 employed an unheated build surface of blue painter's tape. 


\subsubsection{Mold Assembly}

In preparation for molding, the plexiglass was cut into four $12 \mathrm{inch}$ by 3 inch pieces and arranged into a rectangular shape on the surface of the glass slide. The pieces were sealed in place with caulk and allowed to set for the required three days (Figure 11). The pieces of the aneurysm model were sanded to remove sharp edges or protrusions left from FDM manufacturing, glued together using silicone cement, and allowed to set for one day as the caulk cured, in preparation for the molding process described in Figure 12.

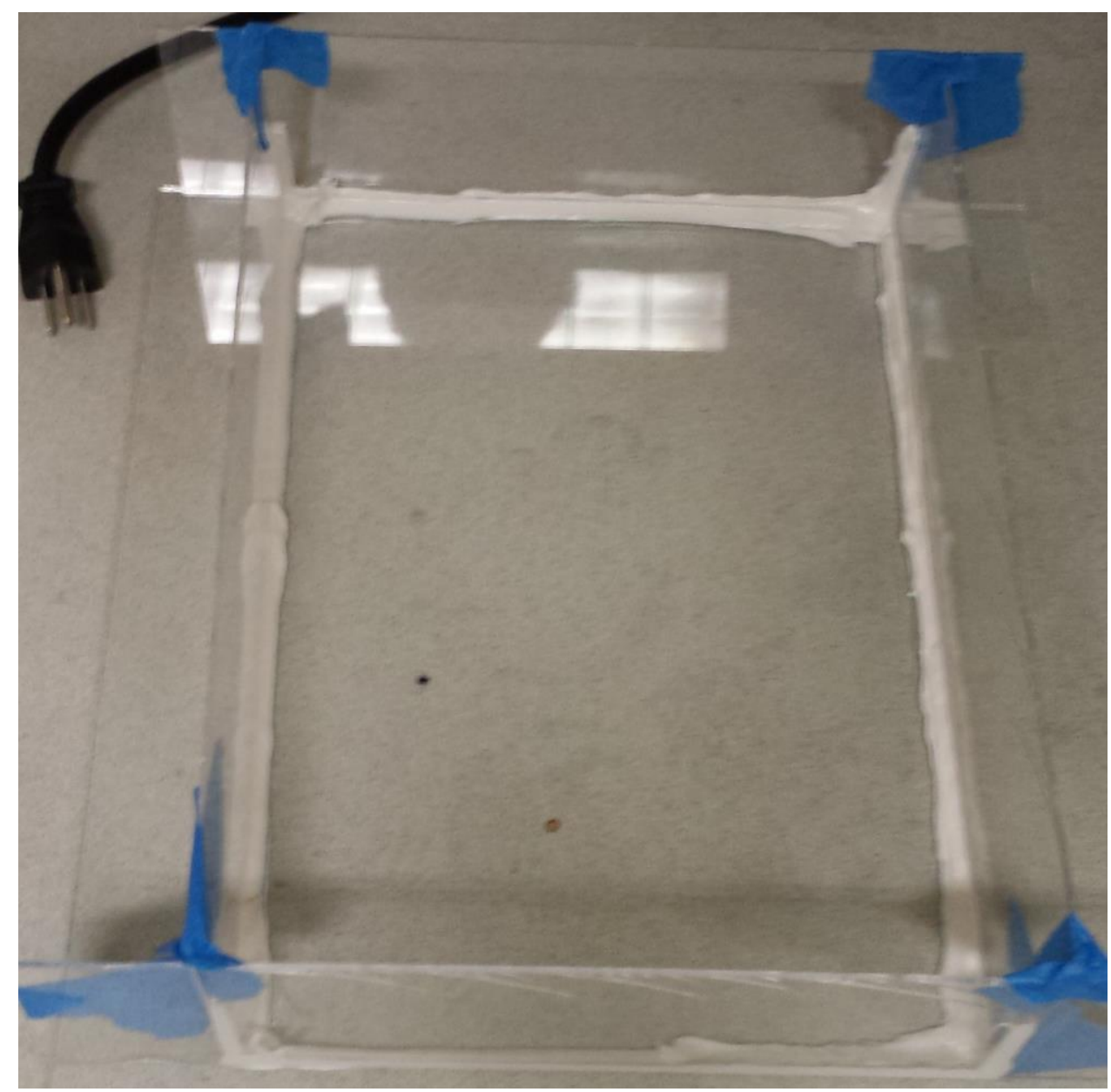

Figure 11: Completed mold featuring plexiglass walls fixed to a glass surface with caulk. This configuration allowed the PDMS mold to set before being fixed to the glass by plasma bonding. 

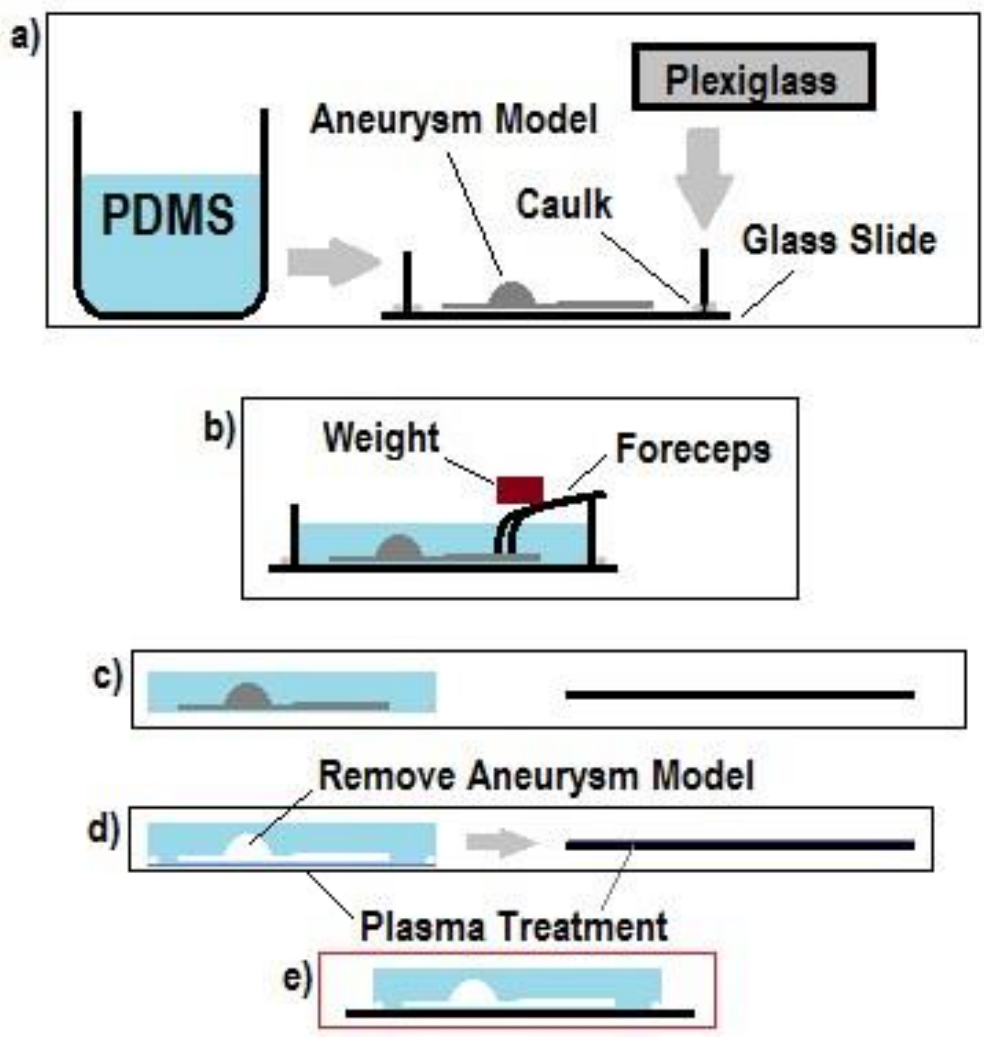

Figure 12: The general steps in construct production include a) construction of the mold, and pouring PDMS, b) allowing the PDMS to set, c) separating the PDMS from the glass slide and plexiglass walls, d) removal of aneurysm model, and cleaning mold, followed by a plasma treatment, leaving e) the final aneurysm construct which was subsequently subjected to a heat treatment described below.

\subsubsection{PDMS Processing}

After the caulk and glue had set, 2.0 kilograms of PDMS were prepared for the mold in four $500 \mathrm{~g}$ batches. For each batch, the PDMS and fixing agent were combined and mixed for 15 minutes (in excess of the required 10 minutes), poured into a $900 \mathrm{ml}$ beaker, and placed in a desiccator which functioned as a vacuum chamber to remove air from the PDMS mixture [31]. The vacuum was pulsed repeatedly until the amount of air in the mixture was low enough to prevent it from overflowing. Finally, the vacuum was 
pumped with the mixture inside for an hour to remove the remainder of the bubbles. The PDMS was poured over the model in the mold and left to set for 24 hours [30].

\subsubsection{Aneurysm Construct Manufacturing}

Once the PDMS had set, the forceps which were used to weigh down the aortic section of the model were removed, and PDMS was injected into the resulting gap with a syringe. This PDMS was allowed to set for one day. After the PDMS had set, the forceps were removed, and a $10 \mathrm{~g}$ batch of PDMS was prepared to back fill the holes left by the forceps. After the filler PDMS had set for a day, the mold was removed from the plexiglass and glass box, and a precision knife was used to make incisions along the outline of the aneurysm model to allow the removal of the parts of this model. The removal of the model yielded four different artifacts worth mention (Figure 13):

1. Ridges marking the different layers of the model left by the FDM process.

2. Infilled joints where PDMS filled the gap between the model components which were printed separately and attached prior to molding.

3. Blocked outlets where PDMS filled the gap between the outlets of the model and the Plexiglass wall.

4. Excess PDMS flaps beneath the underside of the half-vessel model with thicknesses between $0.5 \mathrm{~mm}$ and $3 \mathrm{~mm}$ which resulted from the model lifting slightly off the glass. 


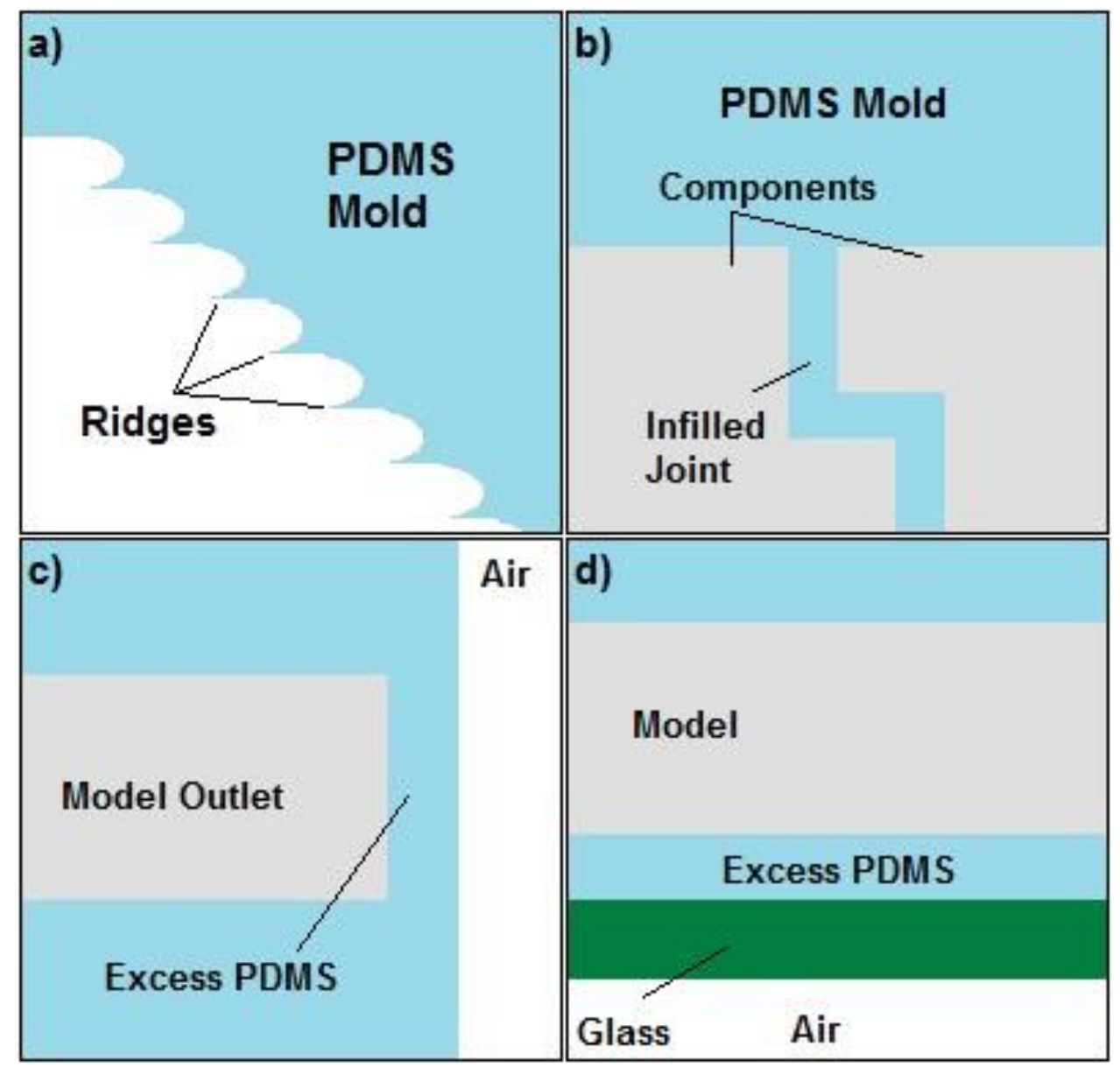

Figure 13: Manufacturing the aneurysm model, and producing a mold resulted in four types of artifacts, including a) ridges in the PDMS left by the edges of individual layers from the FDM process, b) infill of the joints between components, leaving a flap which needed to be removed by trimming, c) excess PDMS filling the gap between the model outlets and the mold wall; which required removal to allow flow through the mold, and d) excess PDMS flaps underneath the half vessel model which was removed prior to flow visualization.

The ridges left by the model were allowed to remain since removing them by painting PDMS over the cavity to smooth it out would risk pooling in the cavity. But the excess PDMS which filled gaps between model components, blocked outlets, or formed flaps was cut away with a precision knife to ensure a smooth path for fluid flow after plasma bonding. Once the excess material had been cut away, the PDMS and the glass 
slide were prepared for plasma bonding. First, the plate was washed with deionized water and wiped down with isopropyl alcohol (IPA). Then debris was removed from the PDMS construct using scotch tape. Both the plate and the construct were treated using the plasma bonding machine to allow strong covalent bonds between the PDMS and glass. After the glass and PDMS were pressed together, polyvinyl chloride tubing was inserted to the inlet of the construct and the model was heat treated for 2.5 hours at $60{ }^{\circ} \mathrm{C}$, and allowed to furnace cool to reduce the risk of having the glass slide shatter due to thermal shock. This temperature was lower than the $75^{\circ} \mathrm{C}$ typically used for microfluidic chips. This was necessary to prevent the PVC tubing from being damaged during the heat treatment. Typically, microfluidic devices are heat treated for about 5 minutes on a hot plate. However, to compensate for the low temperature as well as the high mass of the construct, a relatively long time was chosen [30]. After heat treatment, $10 \mathrm{~g}$ of PDMS was prepared in accordance with the protocols described above and injected into the inlet to fill the space between the tubing and the construct. The purpose of this was to minimize leakage during the imaging trials.

\subsection{Full Vessel Construct}

\subsubsection{FDM Manufacturing}

In preparation for manufacturing, the full vessel model was split into six pieces to save space on the build surface, and to avoid need for support material. Additionally, a connector was prepared in SolidWorks to allow the $3 / 16$ inch $(0.476 \mathrm{~cm})$ inner diameter PVC tubing to attach to the aneurysm model. The connector was built around a quarter circle with a $4 \mathrm{~cm}$ radius. Circles were drawn perpendicular to the quarter circle at 
regular intervals. The base of the connector, which connected to the aorta was a $2 \mathrm{~cm}$ diameter circle, and the interface with the connector had a 3/16 inch $(0.476 \mathrm{~cm})$ diameter. The final shape of the connector was akin to a cornucopia from Thanksgiving decorations. This design allowed the tubing leading from the pump to connect to the top face of the construct without bending the tubing within the PDMS construct, a design feature intended to prevent tubing from touching the walls of the construct, leaving a weakness in the mold.

This "cornucopia connector" and the pieces of the full vessel model were FDM manufactured on a Type A Machines Series 1 , at $200{ }^{\circ} \mathrm{C}$ using water soluble polyvinyl alcohol (PVA) filament (Figure 14). The build surface was covered with blue painter's tape, and was unheated. After printing, the components were glued together with epoxy, and temporarily bound with dark annealed steel wire and blue painter's tape for about 24 hours to allow the epoxy to set. The aneurysm model parts were printed without need for support material. However, the "cornucopia connector" was printed using the "rough" support material setting, which applied a scaffold on overhangs exceeding $45^{\circ}$ from vertical. The FDM machine used featured a single extruder system, so the same material was used for the support scaffold and the part. Both the connector and the aneurysm parts were printed with $33 \%$ infill and a $1 \mathrm{~mm}$ skin. The empty space left by the infill was intended to allow faster dissolution of the part. Another consequence of the limited infill was that the buoyancy of the part on uncured PDMS increased compared to the solid material. 


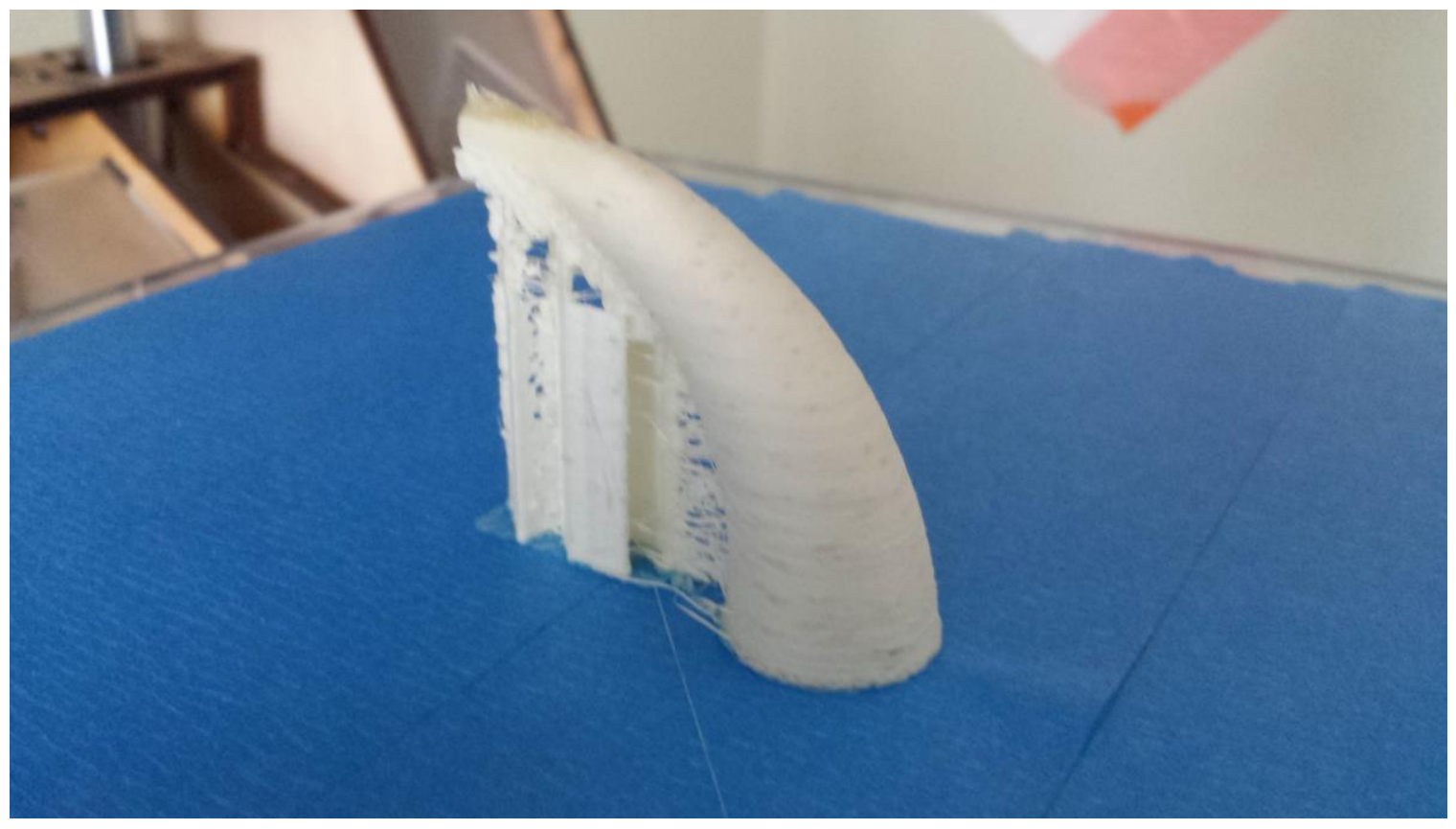

Figure 14: “Cornucopia Connector" after printing had finished. The print featured support material to prevent warping during print. Support material was removed before the molding process.

\subsubsection{Mold Preparation}

A plexiglass-glass mold was prepared in a manner similar to that used for the half-vessel model. The main difference was that the plexiglass walls were arranged in a triangular configuration to allow the PDMS to be poured deeper, which was necessary for the larger full vessel model. The plexiglass walls were secured in place with blue painter's tape, and caulk was applied at the edges and corners of the mold. After the caulk had set at the interfaces between the plexiglass pieces, and at the plexiglass-glass interfaces, $1.0 \mathrm{~kg}$ of PDMS was poured over the full vessel model. The full vessel model was allowed to float on the PDMS until it had set the next day, securing it in place (Figure 15). Once the first layer of PDMS set, the tubing was attached to the "cornucopia connector." and $1 \mathrm{~kg}$ of PDMS was poured over the remainder of the model, submerging it completely. 


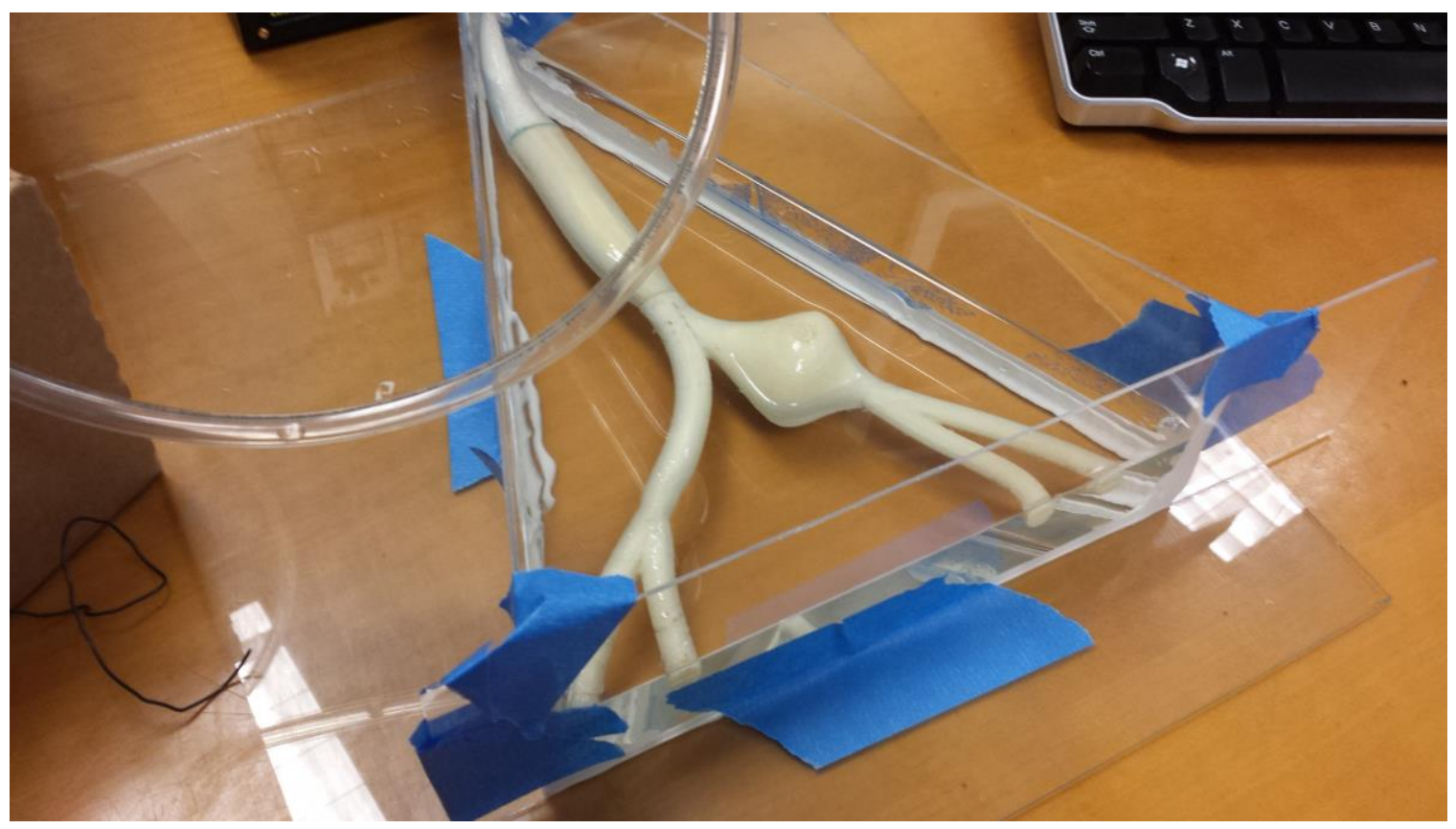

Figure 15: Full Vessel Mold half filled with PDMS. At this stage, the PDMS was allowed to set so the model would be secured in place, preventing buoyant forces from lifting the model out of the mold once the model was completely submerged in the subsequent layer.

Pouring the PDMS in layers allowed it to be suspended within the PDMS without need for additional support. After the final layer of PDMS had set, the PDMS construct was peeled away from the glass and plexiglass (Figure 16). Excess PDMS at the outlets was cut away with an $\mathrm{x}$-acto knife, the tubing was removed from the connector, and the construct was dropped into a trashcan filled with water to dissolve away the PVA and leave a cavity in the shape of the vasculature (Figure 17). After four days of soaking in water, minimal dissolution was observed near the inlet and outlets. To increase the dissolution rate, the PDMS construct was heated to between $70{ }^{\circ} \mathrm{C}$ and $100{ }^{\circ} \mathrm{C}$ in a pasta pot over a gas stove. The dissolution rate increased significantly when this change was introduced. However, this method was abandoned in favor of "baking" the model in a casserole pan filled with water in a gas oven at $93.3^{\circ} \mathrm{C}\left(200^{\circ} \mathrm{F}\right)$, which allowed more 
complete submersion of the construct. While heating the construct in water significantly increased the dissolution rate, this remained a slow process requiring the construct to be "baked" for 4 to 8 hours each day over the course of a week. After a week, the mold was left in a sink filled with water for 24 hours per day for 14 days, which was periodically reheated to $120^{\circ} \mathrm{F}\left(49^{\circ} \mathrm{C}\right)$, due to concerns about residue and discoloration caused by the baking process. The aneurysm model was not completely dissolved, so work on the full vessel model ceased.

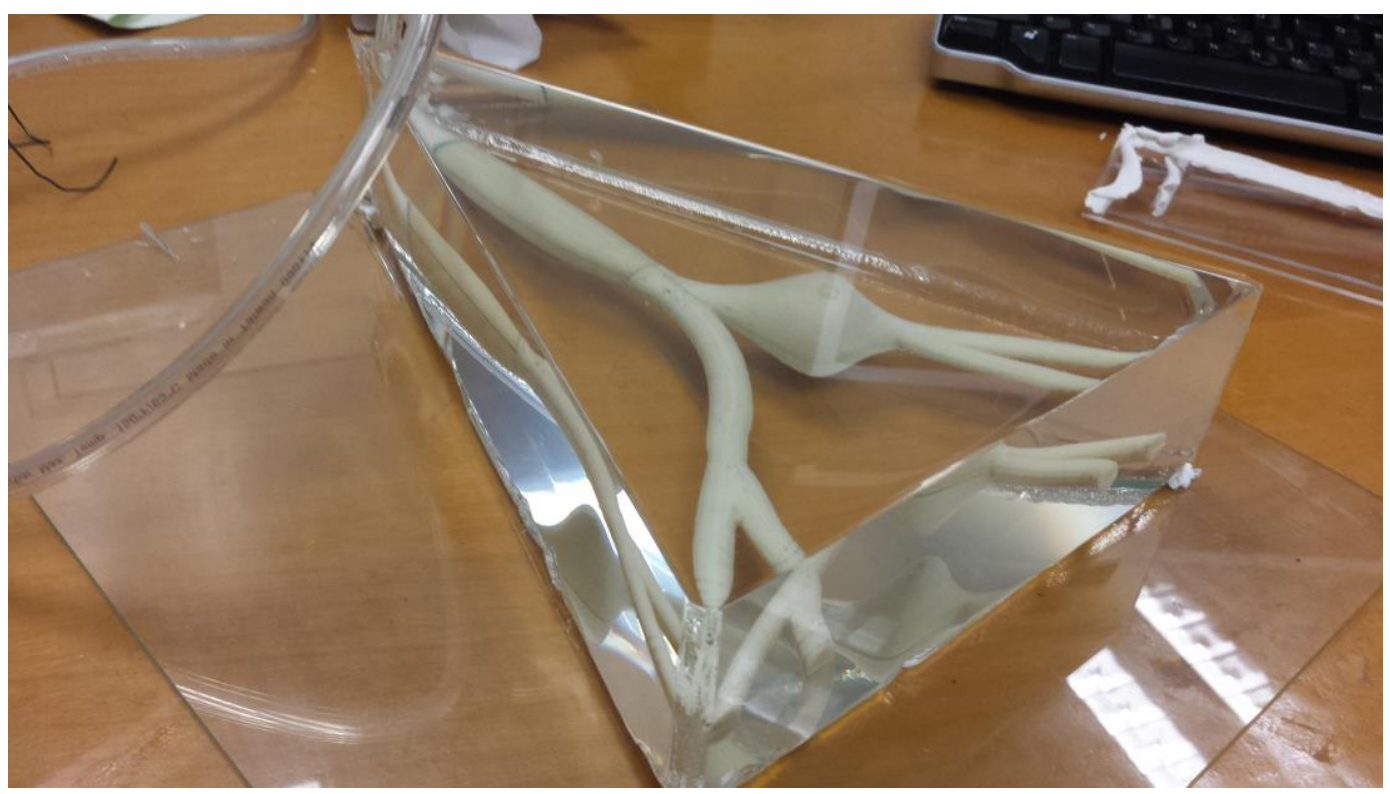

Figure 16: Full Vessel construct after PDMS had set, and plexiglass sides were removed. 

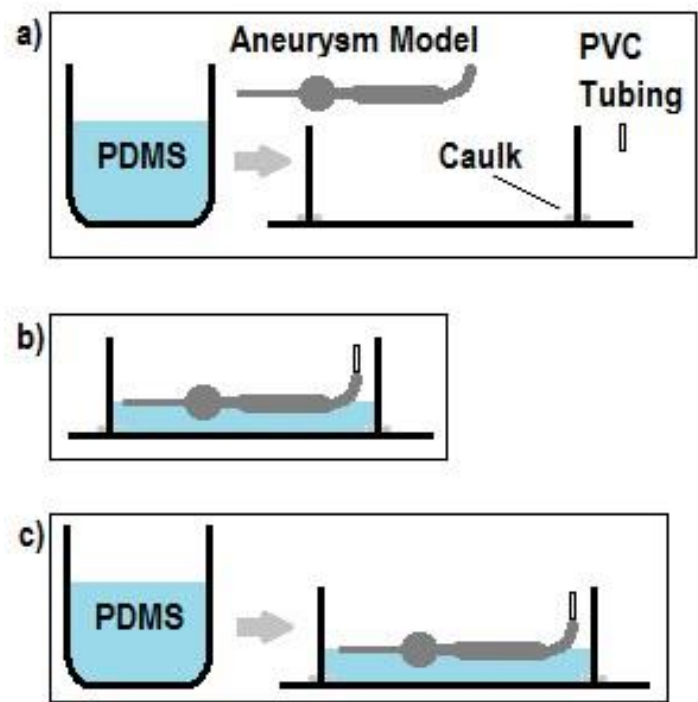
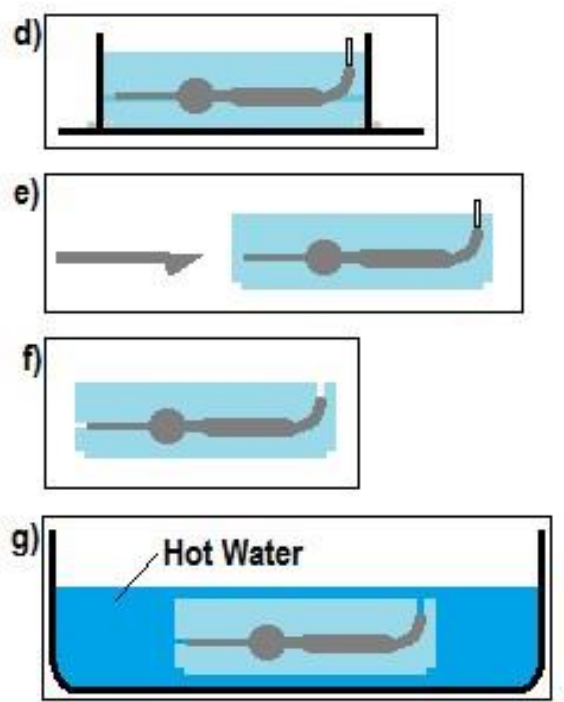

Figure 17: The general steps involved in the production of the full vessel include a) mold assembly and pouring half the PDMS into the mold, b) allowing the PDMS to set, c) pouring in the second half of the PDMS, d) allowing the final layer of PDMS to set, e) cutting excess material away from the outlets, and f) dissolving the PVA by submerging the mold in hot water.

\subsection{Imaging Fluid Flow}

\subsubsection{Particle Imaging Velocimetry}

Particle Imaging Velocimetry (PIV) is a widely used technique for the characterization of fluid velocity profiles, particularly in microfluidics. This technique employs fluorescent beads microns in diameter that are typically tracked by a microscope, which records snapshots in rapid succession. The addition of beads increases the viscosity of the fluid, but the magnitude of this effect depends on factors such as volume fraction of particles, particle size, and shear rate [45]. The positional changes of each particle between frames recorded at a known time interval allow for the velocity of each particle to be determined as well as that of the fluid surrounding each particle. To obtain the most accurate reading possible, the displacement of the center of each particle is to be tracked, with uncertainty 
about the position of the center of the particle being a result of the limited number of pixels occupied by each particle in each image. To prevent particles from significantly altering fluid flow, it is necessary to use particles that are small compared to the hydraulic diameter of the channel. However, the particles also need to be large enough to be visible against the background noise. Due to their small size, random Brownian motion causes random displacements of particles, introducing noise into velocity measurements which can be modeled using a Gaussian probability function [24].

\subsubsection{Dye Imaging}

Traditionally, fluid flow in microfluidics is done by particle imaging velocimetry analysis. In the initial trails, imaging was conducted on a construct with a depth of about $2 \mathrm{~cm}$, exceeding the $1.3 \mathrm{~mm}$ focal length of available microscopes typically used in PIV. Therefore, imaging of fluid flow was conducted using a 13 megapixel camera built into a Samsung Galaxy S4 smart phone at 30 frames per second. Once water flow through the model had been established, red food dye was injected instead of fluorescent beads. In each trial, clear water was pumped at a constant flow rate of $2.26 \mathrm{~L} / \mathrm{min}$ into the aneurysm model. The glass side was faced upward towards the camera to minimize the number of air bubbles would be trapped inside the construct. Prior to imaging, the outlet side was lifted upward to allow air bubbles to escape. Once the construct was completely filled with water, $1 \mathrm{~mL}$ of dye was injected into the inlet of the pump using a syringe, and the movement of the dye through the aneurysm was tracked between the times the dye initially entered the aneurysm until it had completely cleared out of the aneurysm (Figure 18). Introducing the dye close to the inlet allowed it to be transferred into the 
aneurysm construct before it had thoroughly mixed with the water. Additionally, a white container lid was used as a background to allow easier image processing. For this analysis, six imaging trials were recorded for comparison to a COMSOL simulation (Appendix D).

A two foot high table-like fixture was used to hold the camera in place while imaging. The camera lens was placed face down on a plexiglass surface so that it could image the model below from a stationary position. In addition, white paper towels were used as a background, sandwiched between the PDMS construct and a pane of plexiglass. This configuration prevented dyed water from pooling underneath the aneurysm model on the paper towels after flowing out of the construct. Still photographs of the aneurysm and a ruler were recorded separately from the film to allow length scales to be determined during image processing later. 


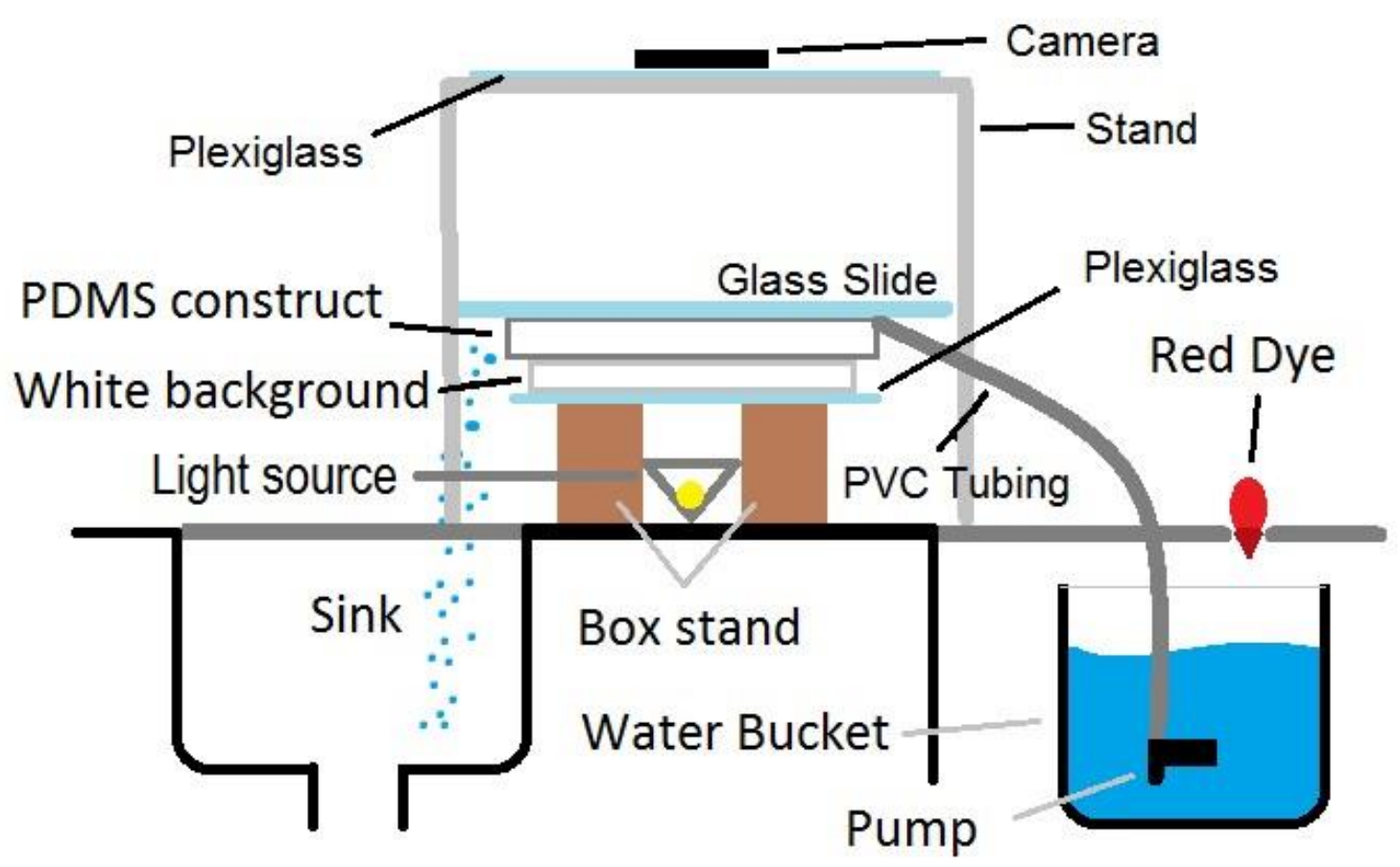

Figure 18: Schematic of imaging setup. Clear water was pumped through the construct until a flow profile was established. Next, dye was introduced to the inlet and its flow patterns were captured by the camera before draining into a sink.

Initially, ambient lighting from the fluorescent lights in the lab was allowed to reflect off the construct during imaging. This resulted in false positive pixels resulting from light passing through the dye and reflecting off the background and passing the threshold settings used during image processing. To resolve this issue, a backlight was added to the setup, and the fluorescent ceiling lights were turned off to prevent light passing through the dyed water from reflecting off the white background and introducing false positive readings. 


\subsubsection{Image Processing}

The films from each trial were separated into individual still images starting shortly before the dye entered the aneurysm and ending shortly after the dye began to pass through the aneurysm. Next, these selected frames were sent to ImageJ for processing. In ImageJ the images were scaled according to a ruler included in the recordings and then a threshold was introduced to select a "critical" dye concentration to allow approximate velocity measurements. Thresholding for trials involving ambient lighting was conducted in Hue-Saturation-Brightness (HSB) mode or YUV mode. The settings selected were specific to the lighting conditions in an individual recording, and therefore were adjusted between imaging trials. It should be noted that the chosen threshold was uniform regardless of the thickness of the section of the model that the dye was passing through, or dilution which occurred as the dye filled more of the construct. Therefore, the measured position of the dye front may have varied based on vessel thickness or dye concentration in addition to fluid velocity. The parameters selected to pass the threshold in HSB mode included a hue between 0 and 23 (the red part of the spectrum to match the dye color), saturation (a measure of pixel intensity) between 235 and 255 to eliminate clear water and noise from the white background, and brightness values from 50 to 255 (dark grey to white) were allowed to pass, eliminating noise from the unlit areas in the images. In recordings analyzed in YUV mode, Y-values (a measure of pixel intensity) between 75 and 255 were passed, U-values (a chrominance component) between 0 and 100 were passed, and V-values (a chrominance component) between 192 and 255 were passed. Thresholding made it possible to select similar dye concentrations between images within the same recording to obtain velocity measurements. Once the dyed water 
was selected in ImageJ, coordinates denoting the position of the front of the dye were recorded in Microsoft Paint. The change in position of the dye front between frames was recorded, allowing approximate velocity measurements to be determined for the intervals between frames.

\subsubsection{Image Analysis}

Each thresholded image was uploaded into Microsoft Paint, where a set of three coordinates for each the dye front was obtained (Figure 19). For each trial, the images were scaled in terms of pixels per meter to allow direct velocity comparisons between trials. The scaling factor ranged from about 3800 to 7300 pixels per meter. The coordinates of the dye front in each image was recorded for analysis in Excel. Once recorded, the coordinates of the dye fronts were scaled and a coordinate transformation function was applied to match the image the dye fronts to their equivalent locations in the COMSOL coordinate system (Figure 20). The cluster of dye front locations from all imaging trials, except for Run 5 which suffered a translational error which placed some recorded points outside the aneurysm model in COMSOL, were used to generate a bestfit sixth order polynomial function which provided an expected maximum velocity streamline followed by the dye, which was later used to compare COMSOL predictions

of the velocity with empirical trials. The locations of each dye front in each imaging trial were recorded to produce path-lengths followed by the dye in each trial (Figure 21). Since the timing of the introduction of the dye was not synchronized between trials, pathlengths were used to obtain the closest direct comparisons possible. The distance between consecutive dye front locations and the frame rate were used to produce average 
velocities for those intervals. This average velocity was assumed to be the instantaneous velocity at the mid-point between recorded dye-front locations. These values represented the expected maximum velocity for each interval, as it was assumed the maximum velocity streamline would determine the distance traveled by the dye. The recorded velocities were compared to the velocity measurements predicted by the COMSOL simulation.

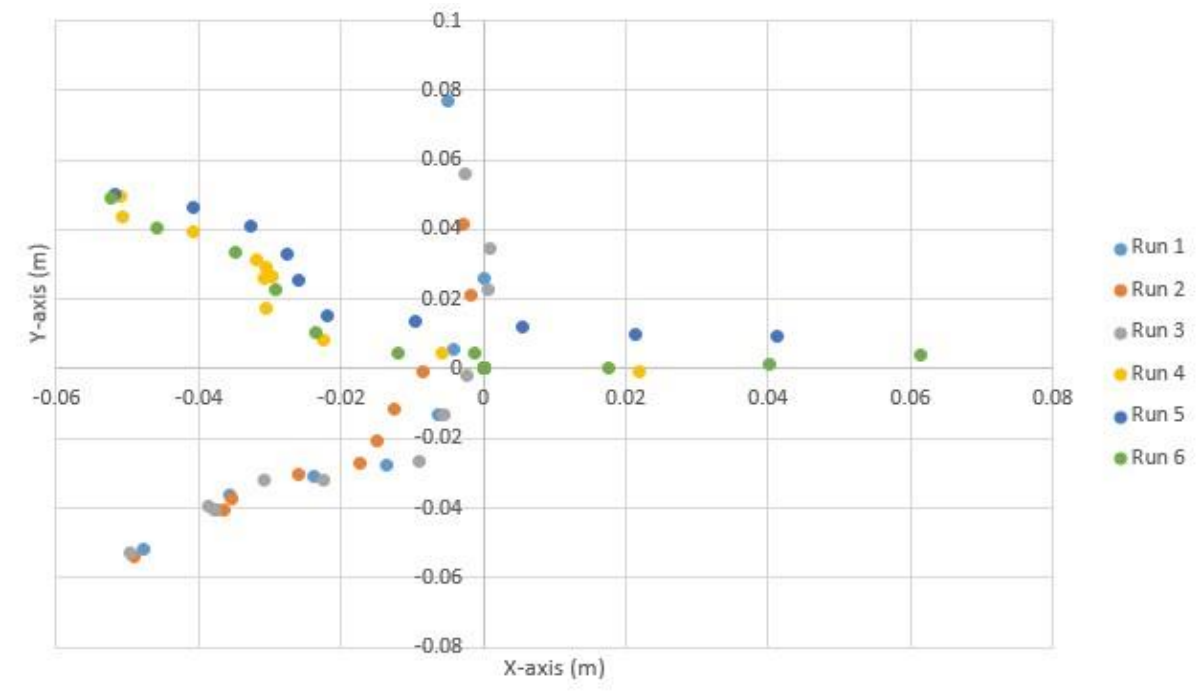

Figure 19: Once the coordinates of the dye fronts were recorded, the scaling was adjusted to convert pixels to meters. However, it was necessary to convert these positions into the COMSOL coordinate system to generate a streamline model, which was used to select point evaluations in COMSOL that would correspond to the motion of the dye in the images. 


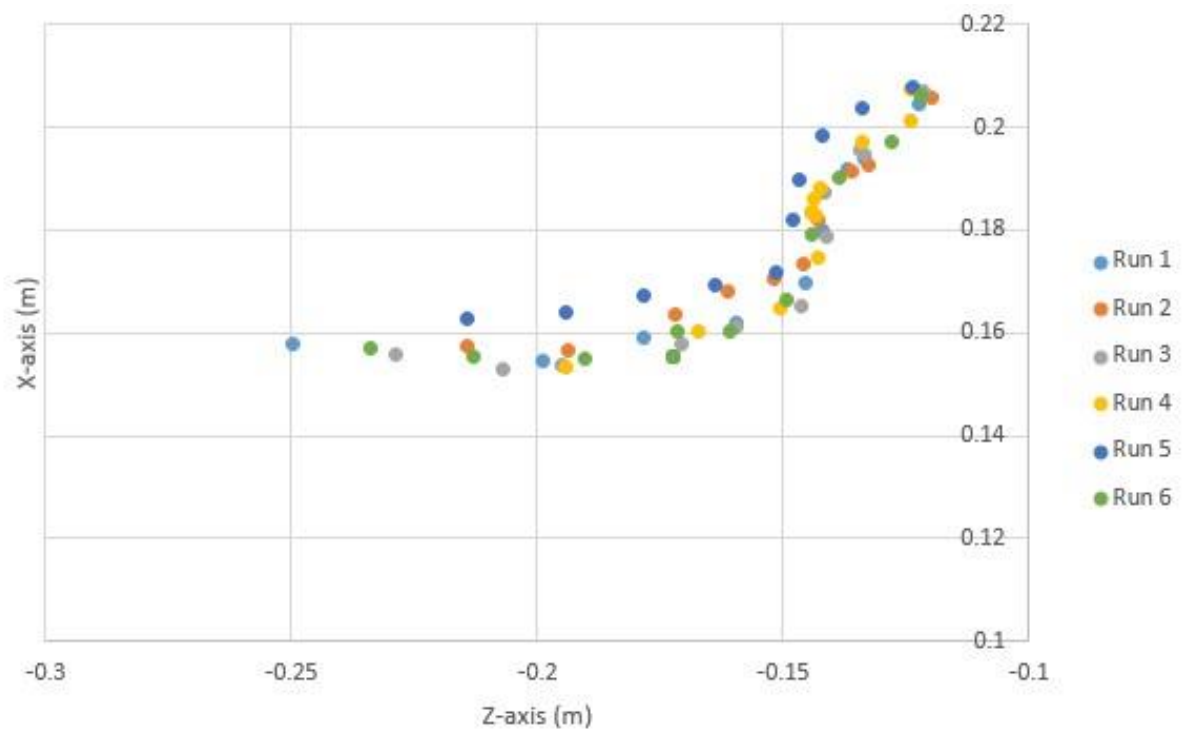

Figure 20: The coordinate transformation converted the recorded dye front positions into the coordinate system used in COMSOL. However, Run 5 (dark blue) was dropped for the purposes of creating the streamline model, because a translational error which shifted the data points in the positive y direction, outside the aneurysm model in COMSOL. Data from Run 5 was still used to obtain velocity measurements which should not have been affected by the translational error.

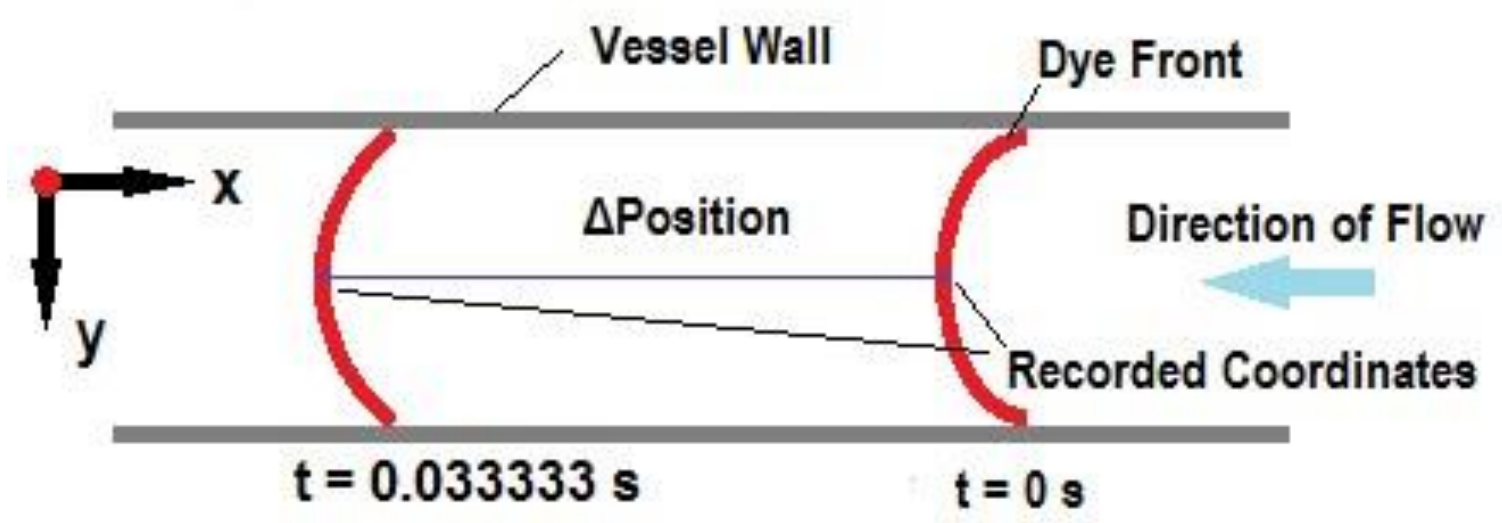

Figure 21: A schematic of the data acquisition techniques used to extract velocity measurements from the dye imaging trials. 


\subsection{Half Vessel COMSOL Validation}

In preparation for COMSOL validation, the volumetric flow rate through the pump was recorded by taking the time required to fill a 1.0 liter beaker. The resulting flow rate was converted into a velocity by dividing the volumetric rate by the cross sectional area of the inlet at the aorta. The resulting velocity was input to the COMSOL simulation. Water fluid properties were used, therefore a viscosity of $1.0 \mathrm{cP}$, and a density of $1000 \mathrm{~kg} / \mathrm{m}^{3}$ were used. The inlet velocity to the Aorta was assumed to be a uniform $0.2397 \mathrm{~m} / \mathrm{s}$ flow, ensuring the volumetric flow rate matched that of the pump used in the empirical study (2.258 L/min). A Reynolds number of about 3200, indicating transient behavior, was computed based on the fluid properties, aortic geometry, and flow rate (Appendix A). Laminar flow behavior was assumed in the simulation so that viscous and inertial effects would be accounted for in the solution. The outlets at the internal and external iliac arteries were assumed be at a pressure of $0.0 \mathrm{~Pa}$. Finally, the flat surface along the $\mathrm{x}-\mathrm{z}$ coordinate plane of the aneurysm model was assumed to be a no slip boundary condition, matching the condition at the glass surface of the empirical model. A mesh refinement study was conducted under the General Physics setting using pre-sets ranging from Finer to Extremely Fine (Table 3). 
Table 3: Half Vessel Mesh Statistics

\begin{tabular}{lrrrr}
\hline Mesh Setting & $\begin{array}{c}\text { Element Type } \\
\text { Tetrahedral }\end{array}$ & Triangular & Edge & Vertex \\
& 43330 & 14676 & 1622 & 337 \\
\hline Finer & 124468 & 28342 & 2486 & 337 \\
Extra Fine & 477900 & 70530 & 4375 & 337 \\
Extremely Fine & & & & \\
\hline
\end{tabular}

\subsubsection{Data Processing and Analysis}

The general steps in processing data from the dye imaging trials are as follows:

1. Record dye front positions frame-by-frame, and adjust scale to convert from pixels to meters.

2. Apply coordinate transformation to convert image coordinate systems to COMSOL coordinate system.

3. Generate model of maximum velocity streamline through aneurysm using dye imaging data.

4. Generate point evaluation arrays in COMSOL based on model.

5. Evaluate velocity between consecutive pairs of frames in each recording, and midpoints between consecutive dye front positions.

6. Compute path lengths for each inter-frame midpoint in empirical trials.

7. Select high velocity point evaluations, and compute path length for each point evaluation.

8. Compute areas between COMSOL and empirical plots using trapezoidal rule, and divide result by area under curve in COMSOL to obtain an area fraction 
9. Conduct one sample, single tailed t-test to determine whether there was a significant difference between recorded area fractions and the threshold 5\% area fraction.

10. The t-test failed to demonstrate a significant difference, determine $95 \%$ confidence interval for average area fraction. A confidence interval with an upper bound area fraction that is less than $5 \%$ would be taken to indicate practical equivalence between empirical trials and the COMSOL simulation.

The conversion from the image coordinate system after scaling to the COMSOL coordinate system was accomplished with the aid of Equations 2.1 and 2.2 shown below:

$$
\begin{gathered}
z_{c}=-x_{p} \cos \alpha+y_{p} \sin \alpha-z_{b} \\
x_{c}=x_{p} \sin \alpha-y_{p} \cos \alpha+x_{b}
\end{gathered}
$$

Where $z_{c}$ and $y_{c}$ represent the coordinates in the COMSOL model, $x_{p}$ and $y_{p}$ represent the coordinates of a point in the image (after conversion from pixels to meters), $z_{b}$ and $x_{b}$ represent the position of the bifurcation in COMSOL, $\alpha$ represents the angle of offset between the image and the COMSOL coordinate systems. The angle, $\alpha$, represented the rotational offset between the coordinate system of the images and the coordinate system in COMSOL. Rotational and translational adjustments were made based on the positions of the bifurcations in COMSOL and in each of the images. Using distinctive features on the model was necessary since there was no clear way to perform the coordinate transformation based on the origins of the coordinate systems. Measuring the distance between the CIA bifurcation inferior to the aneurysm and the aorta bifurcation allowed 
the scaling error to be quantified when compared against the measured distance between these features in the COMSOL model.

The polynomial function was describing the maximum velocity streamline was used to generate point evaluations at $2 \mathrm{~mm}$ intervals along the $\mathrm{z}$-axis (anatomically superior to inferior) on the COMSOL model (Figure 22). This best fit polynomial function was generated based on the positions of the dye fronts in five of the six empirical fluid flow visualization trials. This function was $2 \mathrm{D}$ since it was created based on data collected from the images. However, the model in COMSOL used a three dimensional coordinate system, so the y-axis in COMSOL was ignored in the coordinate transformation. At each point along the streamline, a linear array two or three data points depending on vessel thickness was created in the y-direction. Therefore, once the points were drawn in the COMSOL model, an array was drawn, which consisted of two or three points depending on vessel thickness separated by $1 \mathrm{~mm}$ intervals along the orthogonal axis to the plane of the image, with the first located $1 \mathrm{~mm}$ below the flat surface of the half-vessel model, and the remaining points penetrating deeper into the aneurysm model. In Microsoft Excel, the maximum velocity values in each array were recorded based on the assumption that the limiting factor on the position of the dye front in any given image was the streamline of maximum velocity. A single point was generated within the aorta to serve as a common origin for each trial, allowing direct comparisons of the path lengths to be made. The point evaluations along the polynomial streamline were used to create a plot describing the change in velocity with path length. 


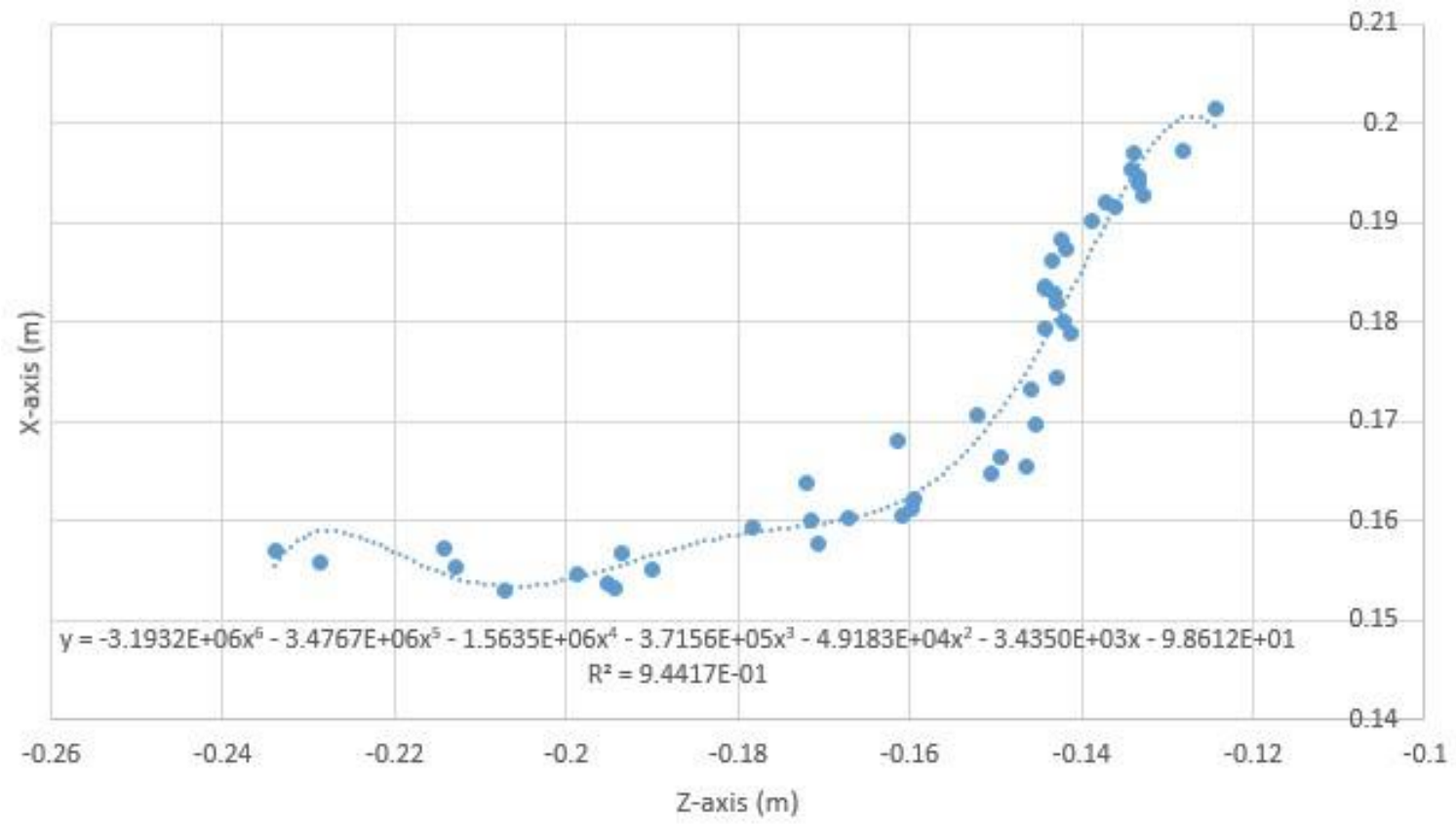

Figure 22: A sixth order polynomial model of the maximum velocity streamline was generated based on the positions of the dye fronts in the images. This model was used to generate point evaluations of the fluid velocity within the COMSOL model of the aneurysm. The function exhibited undesired curvature for $\mathrm{z}-$ values less than -0.213 , located within the aorta. These point evaluations were omitted from an analysis of the velocity profile.

For the empirical data, it was assumed that the average velocity over an interval between frames was the same as the instantaneous velocity at the midpoint between those frames, making it possible to plot the change in velocity with path length for each trial. To compare the velocity profiles quantitatively, Riemann sums using trapezoidal rule were used to calculate the area in between the COMSOL predicted plot and the empirical plots. It was necessary to use linear interpolation to ensure that the limits of each plot were matched with the COMSOL simulation, and to break up each Riemann sum in cases where the plots intersected to ensure the area between the plots was properly captured. By analyzing the data in this way, empirical plots closely matching the COMSOL plot would produce an area closer to zero than a plot that poorly fit the COMSOL predictions. It should be noted that with any error at all, a positive value for the area between the plots 
would be calculated. These calculated areas were input to statistical analysis as a fraction of the total area under the COMSOL Riemann sum for each trial. A one sample, one sided t-test was used to determine whether there was a significant difference between the empirical plots and the COMSOL plot. Since any discrepancy would result in a positive area for all empirical trials, it was not possible for the errors to cancel and center on a hypothesized mean value of zero difference in area between the empirical and COMSOL plots. For this reason, a small positive hypothesized value of $5 \%$ of the area of the COMSOL Riemann sum was used, and the one sided t-test was used to determine whether the average value of the empirical measurements were significantly larger than that value. Additionally, a 95\% confidence interval for the area fraction was generated.

\subsubsection{Intra-Aneurysmal Flow}

The dye imaging trials did not allow for the velocity of fluid in the lateral aspect of the aneurysm to be assessed. Therefore, analysis of intra-aneurysmal flow was conducted in COMSOL. The inlet and outlets featured the same velocity profiles as the trial above, and the extremely fine mesh setting was used. In contrast with the velocity assessment, the flat X-Z plane featured a symmetry boundary condition. Therefore the model was treated in the same way as the full vessel for this trial. An arrow plot was generated using logarithmic scaling indicating the direction and relative magnitude of the flow field within the aneurysm model. Surface plots were generated to obtain the velocity and shear stress profiles within the aneurysm model. 


\subsection{Half Vessel Results}

\subsubsection{Comparison of Empirical Velocity Measurements to COMSOL}

Introducing food dye into water flow through the half vessel model allowed for an empirical evaluation of the velocity of the water through the vasculature. A comparison of the empirical trials to a COMSOL simulation indicated poor agreement between the output velocity values of the empirical trials and the COMSOL simulation. The empirical trials followed similar decreasing trends while the COMSOL model predicted an increasing velocity at the proximal aspect of the streamline, which plateaued and dropped at a path length of about $0.1 \mathrm{~m}$, and then steadily increased towards the distal aspect of the streamline (Figure 23).

Analysis of dye movement through the model would not allow a direct comparison of the flow conditions in the construct and the COMSOL model. Visual analysis of the dye motion through the aneurysm indicated there was rotational fluid flow within the aneurysm, which was corroborated by an arrow plot of the velocity profile generated by COMSOL (Figure 24). The maximum velocity streamline generated from the empirical observations of the dye front motion was found to conflict with the streamline predicted by COMSOL, such that the streamline model was found to follow a path within the aneurysm which was medial to the streamline in COMSOL (Figures 25 and 26). Therefore, point evaluations gathered in COMSOL based on the streamline model, particularly in the distal half of the model, cannot be regarded as representative of the predictions that might come from the maximum velocity streamline predicted by the COMSOL simulation. The sharp drop in velocity at the $0.1 \mathrm{~m}$ path length in Figure 23 
could be attributed to the departure of the streamline model from the jet in COMSOL. By contrast, the surface plot suggests that the velocity plot should have quickly ramped up from about $0.3 \mathrm{~m} / \mathrm{s}$ to about $0.8 \mathrm{~m} / \mathrm{s}$ in the proximal half of the model before steadily declining to about $0.4 \mathrm{~m} / \mathrm{s}$ in the distal half of the streamline.

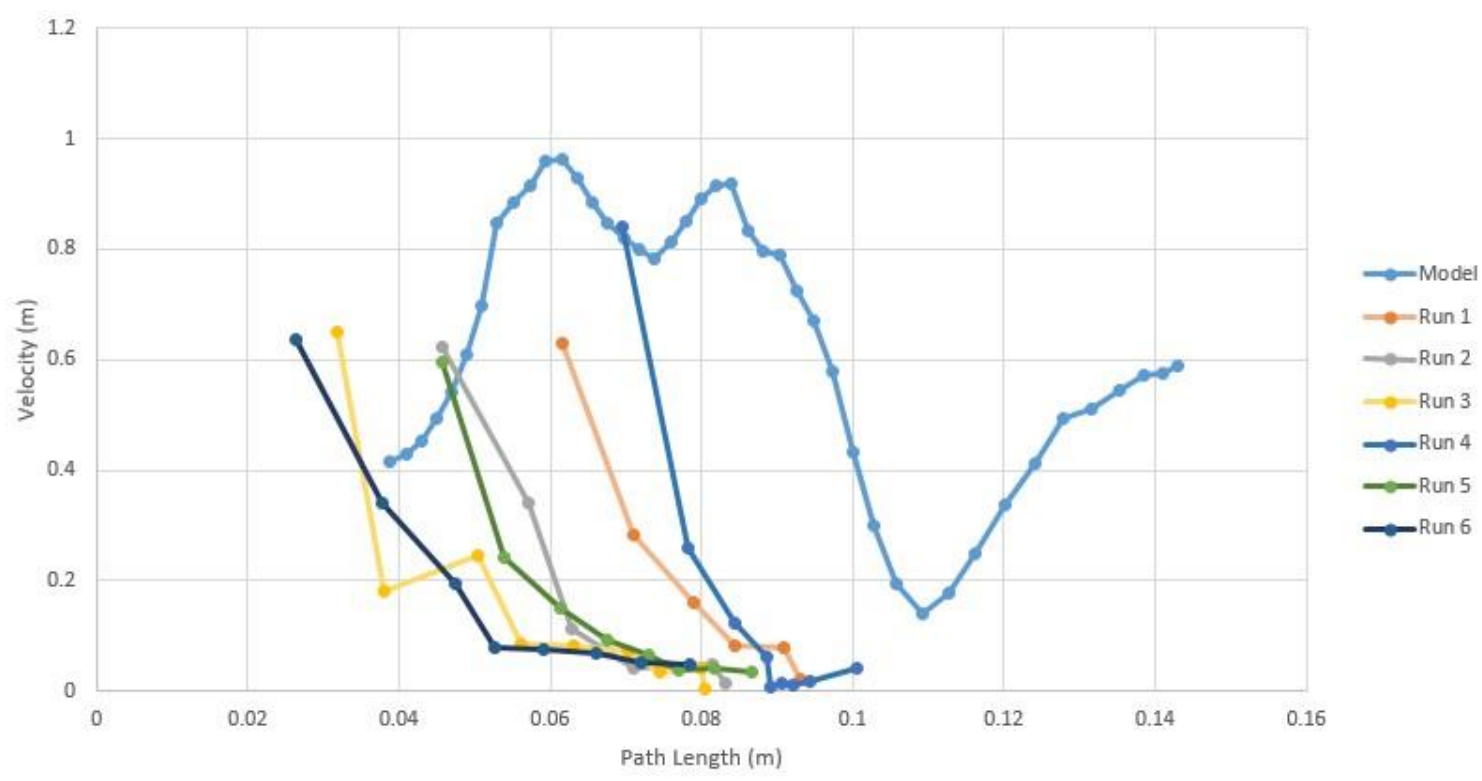

Figure 23: A comparison between the velocity measurements output by COMSOL and empirical measurements. A discrepancy was evident based on a cursory examination of the data. However, the ratio of the area between each empirical plot and the COMSOL plot was generated using trapezoidal rule and identical limits for each comparison were used to obtain a quantitative assessment of this discrepancy. 


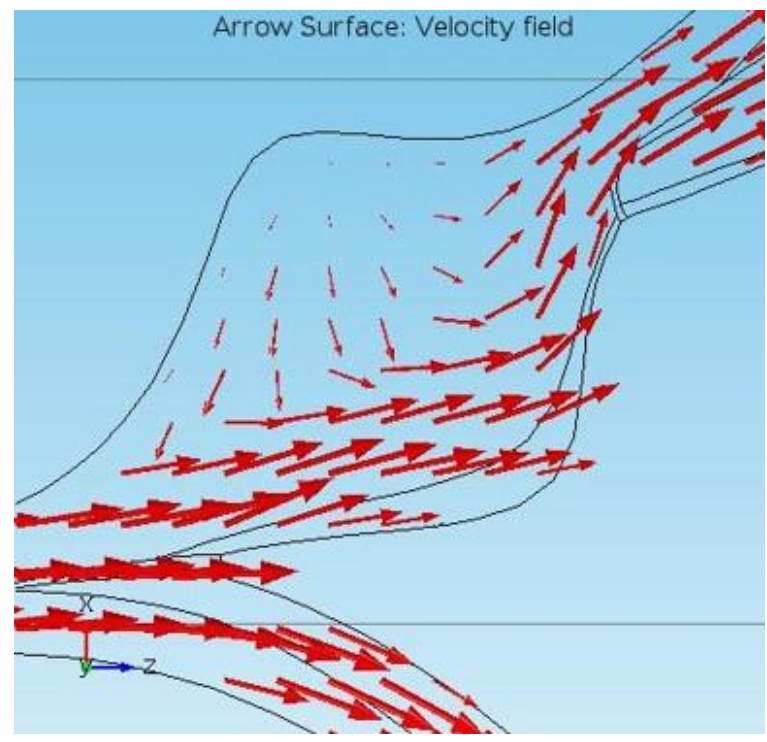

Figure 24: An arrow plot using logarithmic scaling shows the presence of a high velocity jet at the medial surface, and low velocity back flow at the lateral side of the aneurysm, demonstrating rotational flow within the aneurysm.

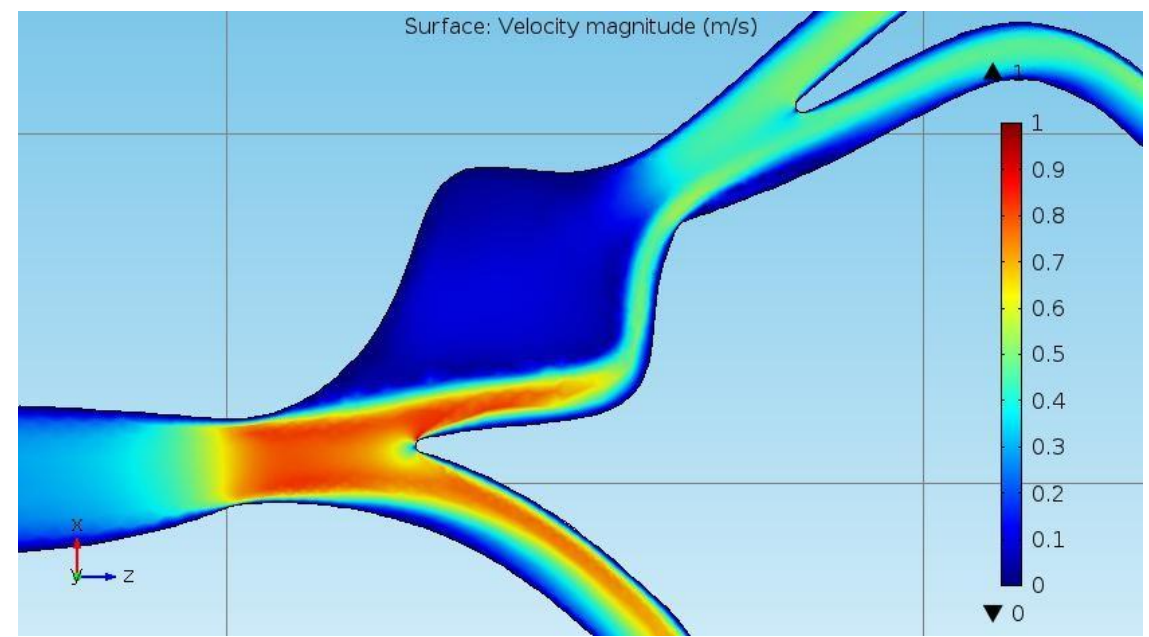

Figure 25: Surface Plot of velocity provides quantitative assessment of intra-aneurysmal velocity profile, revealing the high velocity jet at the medial surface of the aneurysm. 


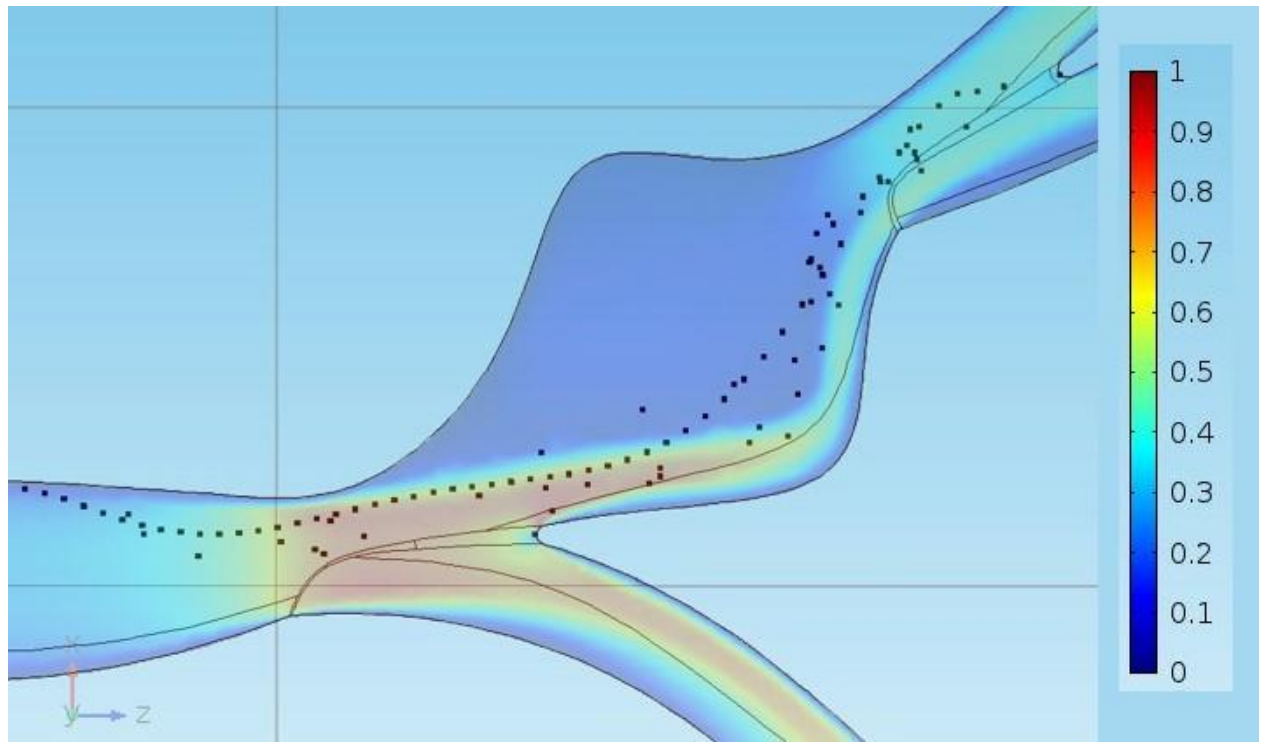

Figure 26: Overlay of velocity surface plot and point evaluations generated in COMSOL, including both the streamline model and the raw data used to generate that model. Roughly the distal half of the streamline model runs slightly medial to the maximum velocity jet through the aneurysm predicted by COMSOL.

\subsubsection{Statistical Analysis}

A one sample, single tailed t-test was conducted to determine whether there was a significant difference between the area fractions computed for the empirical trials and a threshold 5\% area fraction, above which the empirical plots and the COMSOL plot would be considered significantly different. It should be noted that failing to find a significant difference would not necessarily indicate practical equivalence. Rather, empirical data would be considered practically equivalent if the upper bound of the $95 \%$ confidence interval for the area fraction remained below that 5\% area fraction threshold. A result placing the 5\% threshold within the $95 \%$ confidence interval would be considered indeterminate. At a 95\% significance level, a difference between the area fractions calculated based on the empirical trials, and the 5\% threshold was found $(\mathrm{p}=$ $\left.3.61 \times 10^{-7}\right)$. The $95 \%$ confidence interval for the area fraction was found to be $0.714<\mu$ 
$<0.837$. Therefore, the imaging trials conducted in this study failed to demonstrate similarity between the empirical measurements of the velocity profile, and the COMSOL predictions.

\subsubsection{Predicted Vascular Wall Shear Stress}

The model indicated the highest shear stress concentrations at the bifurcations of the vasculature, reaching values as high as about 4.0 Pa. Shear stress values along the vascular walls, particularly at the lateral side of the aneurysm remained close to zero (Figure 27).

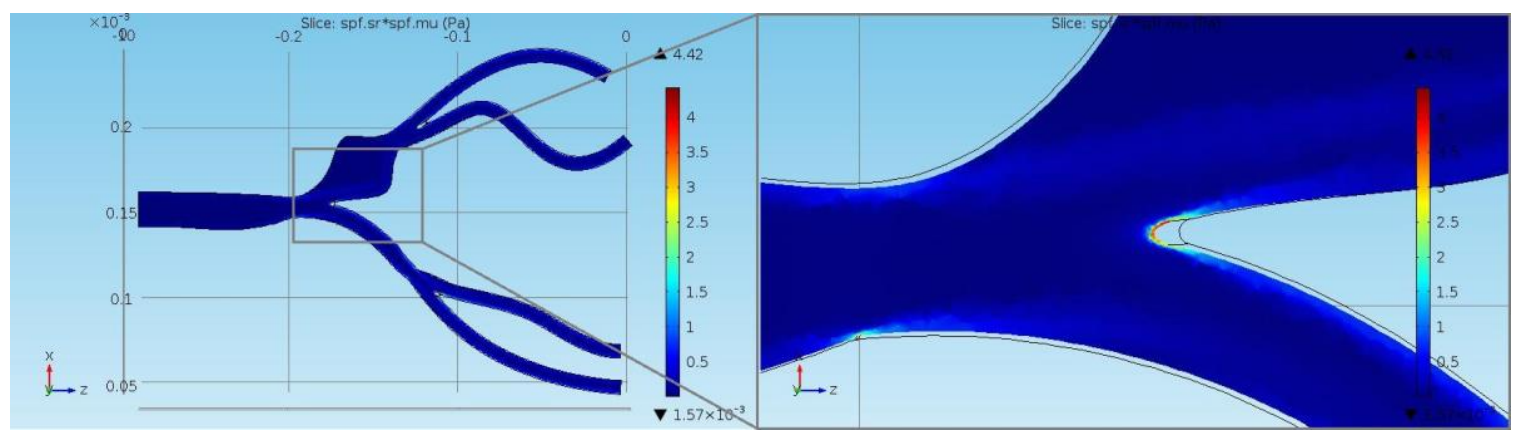

Figure 27: Shear stress slice plot within aneurysm model. The highest shear stress regions are located at the bifurcations of the vasculature.

\subsection{Full Vessel}

The full vessel construct was not completed, due to the slow dissolution rate of the PVA. Therefore, imaging trials were not conducted. 


\subsection{Discussion of Half Vessel Findings}

The dye imaging trials conducted on the half vessel produced maximum velocity measurements that were found to be significantly different from the velocity measurements output from the COMSOL simulation. This most likely indicates a need to modify the experimental methods to obtain more consistent and reliable data.

\subsubsection{Analysis}

\subsubsection{Empirical Dye Imaging Trial}

The most important improvements to the half-vessel model would involve improving control over imaging conditions that may be used to obtain more accurate measurements. Steps to improve the imaging protocol include the selection of a more homogenous background to scatter light, such as a flat white translucent panel in the background which would avoid variations in the light intensity. This would contrast with results obtained in this study as a result of the wet spots and overlapping layers of paper towels. A 2D array of light sources might be used to avoid the light intensity gradient, an artifact of the use of single lamp in this study. Avoiding undesired variation in pixel color and intensity may ensure that subsequent thresholding in ImageJ results in cleaner demarcations between non-dyed regions and dyed regions in the image. Fluorescent dye may be used in future imaging trials to allow easier isolation of a signal from the fluorescent dye against a dark background as the threshold is applied. However, a major issue with the use of dye was the degree of subjectivity involved in interpreting the images. There were instances when a frame might have a small cluster of pixels which passed the threshold filter which were ahead of the group, or where the dye front had a 
flat appearance making it necessary to judge which pixel on the leading edge of the dye front would be counted as furthest point moved by the dye. The need to interpret images in this way may have contributed to observed variability in both the recorded positions of the dye front, and consequently, the measured velocity of the dye. Employing some form of PIV would remove this subjectivity from data interpretation, since individual particles could be identified and tracked by camera. PIV would make it possible to characterize the entire intra-aneurysmal flow field. By contrast, dye tracking only allowed the identification of the maximum velocity streamline in the flow.

Imaging might be conducted in future work by using cameras with higher frame rates such that shorter intervals between frames are used to evaluate velocity profiles. This would improve the "resolution" of velocity evaluations, and reduce error resulting from the assumption that the dye or particles followed a straight path between each pair of frames. The precise choice in frame rate would depend on the expected maximum fluid velocity since controlling the displacement between frames would be a major concern. It should be noted that since the recorded velocity of the fluid is dependent on both image resolution and frame rate, so benefits from improved frame rates diminish as the change in displacement in dye front or particle position between frames approaches the length scale of an individual pixel.

Artifacts of the Manufacturing process, such as seams between sections of the aneurysm model, and excess space left by PDMS which had filled the space ranging from $0.5 \mathrm{~mm}$ to $3 \mathrm{~mm}$ between the space between the aneurysm model and the glass slide represent defects in the geometry of the construct which may have affected the measured velocity profiles in the empirical model, likely resulting in a reduced measured fluid 
velocity compared to a construct free of defects. These artifacts could be minimized by using a machine with a larger build surface to remove the need to split the model into pieces which created the seams, and by ensuring that the flat surface was completely flush with the glass slide to prevent PDMS from filling in space underneath the model.

\subsubsection{COMSOL Simulation}

The polynomial streamline model, which was used to generate the point evaluations through the estimated maximum velocity streamline in COMSOL was based on the output values of a coordinate transformation from the recorded images of the empirical trials. Therefore, the streamline analyzed in COMSOL represents a best estimate of the maximum velocity stream line through the aneurysm based on the movement of the dye through the model in empirical trials. This introduced some uncertainty into the estimates of the maximum velocity of the flow through the aneurysm. Potential sources of error in the generation of this streamline include that subjectivity involved in selecting data points from individual frames, translational or rotational errors introduced in the coordinate transformation, or scaling errors from the conversion from pixels to meters. The scaling error was able to be quantified by comparing estimated distances between the aortic bifurcation and the CIA bifurcation distal to the aneurysm between the COMSOL model and the images. The scaling error never exceeded an absolute value of $2 \%$ and the average scaling error was $0.024 \%$.

The output values of the point evaluations were subject to change with mesh fineness (Table 4). The relatively small average change between the Extra Fine and Extremely Fine settings seems to indicate that COMSOL had nearly converged on the solution. 
Large variability seen in solutions for individual point evaluations might be of concern in studies focusing on individual points in the flow field, but technique used in this study that analyzed Riemann sums of velocity plots would be insensitive to errors from individual point evaluations. It appears that further work with mesh refinement may improve model accuracy, but errors in the COMSOL simulation are unlikely to have prevented the validation of the flow visualization techniques used in this study.

An improvement which may help to close the gap in velocity values between the construct and the COMSOL simulation would be to ensure that the inlet geometry in the construct closely matches that chosen in the simulation. In this study, the proximal end of the Aorta was assumed to feature a uniform velocity profile at the inlet, while the construct featured a thin 3/16 inch inner diameter tube injecting fluid into a conical attachment to the Aorta. This discrepancy may have affected velocity values in the proximal aspect of the aneurysm. Resolving the discrepancy may be accomplished either by modifying the COMSOL model, or the construct to ensure closely matching geometry and boundary conditions. This discrepancy could be resolved by increasing the length of the aorta in the construct to allow the jet from the inlet tube to dissipate into a more uniform flow field across the aorta before entering the aneurysm - the region of interest.

Table 4: Changes to COMSOL Solution with Increasing Mesh Fineness

\begin{tabular}{ccc}
\hline & $\begin{array}{c}\text { Extra Fine } \\
\text { to } \\
\text { Extremely Fine }\end{array}$ & $\begin{array}{c}\text { Finer } \\
\text { to } \\
\text { Extremely Fine }\end{array}$ \\
\hline $\begin{array}{c}\text { Average Percent } \\
\text { Difference }\end{array}$ & 1.12 & -9.86 \\
\hline $\begin{array}{c}\text { Standard Deviation of } \\
\text { Percent Difference }\end{array}$ & 24.9 & 72.0 \\
\hline
\end{tabular}




\subsection{Discussion of Full Vessel Outcomes}

The full vessel model was not completed because the dissolution rate of PVA was too slow for the time constraints of this project. The factors influencing the dissolution rate of the model are as follows:

1. Water temperature

2. Exposed surface area

\section{Convective effects}

\section{PVA skin thickness and infill percentage}

The principle of creating a mold around a water soluble polymer which could be removed to create a cavity of desired geometry was demonstrated to be sound. The PVA was selectively dissolved successfully. However, the dissolution rate remained unacceptably slow, even with water temperatures at $93^{\circ} \mathrm{C}$, close to the maximum value without need to employ a pressure cooker or autoclave to achieve temperatures above $100^{\circ} \mathrm{C}$. Therefore, the latter three factors affecting the PVA dissolution rate would dominate in informing future improvements to the process used in this project. The exposed surface area in this project was limited to the inlets and outlets. An increase in surface area could be accomplished by creating a channel running throughout the interior of the aneurysm model, through which the water could be pumped. This would also allow increase the convective effects contributing further to the dissolution rate. In addition, the skin thickness and infill percentage could be decreased, leaving a more fragile model while allowing faster dissolution. A small scale experiments testing the influence of factors such as water temperature, skin thickness, infill percentage, and exposed surface area on the dissolution rate would be advisable before attempting to construct another full vessel model. Another matter which deserves consideration is the discoloration observed on the full vessel model. The most likely explanation for this would be soot from the gas oven used for the first week of PVA dissolution. 
Another possibility that would allow future groups to evade the necessity of dissolving PVA entirely would be to create two mirror image models of the half vessel similar to those used for the half vessel in this experiment. The half-aneurysm models could be simply peeled away from the PDMS mold. Plasma bonding could be employed to connect the two halves together, leaving a single PDMS construct. Possible complications with this method would include an offset that would not be possible to correct once the two halves are brought into contact because plasma bonding is irreversible. Therefore, a fixture would need to be created to ensure proper alignment of the two half-vessel molds. If connected successfully, the imaging trials would need to be conducted with the inlet facing downward to prevent air bubbles from being trapped in the aneurysm. Therefore, it would be desirable for the outlets to connect to tubing which could carry the fluid into a sink and prevent water from running down the sides of the mold, and interfering with image collection. Finally, a fluid with a similar coefficient of refraction to the mold material would need to be chosen to prevent optical distortion. 


\subsection{Project Goals}

The original project goals were as follows:

1. Can fused deposition modeling be used to construct useful half vessel and full vessel constructs of an iliac artery aneurysm?

2. Can the velocity profile through the aneurysm models be measured experimentally using dye?

3. Was there good agreement between the COMSOL simulations and fluid flow visualization studies?

This study indicated that FDM can be used successfully to produce constructs for both the full vessel and half vessel models. The half vessel model was produced to accurately represent the model used in the computer simulation, although the molding process did leave minor artifacts such as ridges, seams between separate parts of the model, and excess material. Water was pumped through the model, and allowed clear visualization of dye movement through the construct. The full vessel model was successfully printed and set in a mold. However, the dissolution rate of PVA in the model was too slow to allow the aneurysm shaped cavity to be completed for fluid flow visualization trials. Future work on full vessel constructs could focus on increasing the PVA dissolution rate by reducing the skin thickness or percent infill during printing, or by producing two mirror image half-vessel constructs to be combined after plasma treatment. The latter procedure would be risky since the bond would be irreversible.

The imaging trials conducted on the half-vessel model to compare the predicted velocity profile to empirical observations did not successfully demonstrate agreement between experimental values and COMSOL values. However, dye tracking may prove an adequately effective method of flow visualization with changes to the experimental set 
up. The three major issues uncovered over the course of the experiments were 1) lack of control over imaging conditions, 2) low frame rate, and 3) subjectivity in dye front selection. The use of a single light source for the back light resulted in an intensity gradient in the images which prevented dye from being captured in the superior and inferior ends of the vascular construct. The automatic white balance used by the digital camera gave the white background an orange tinge, making it difficult to properly capture the red dye in all frames. The video recording rate of 30 frames per second prevented the dye from being tracked with a high degree of precision; the empirical trials involved the collection of between 7 and 10 frames per run, which followed the movement of the dye front from the aorta through the aneurysm, producing an average change in dye front position of $1.28 \mathrm{~cm}$ between each pair of consecutive frames. A higher frame rate would allow more precise tracking of fluid motion.

These goals were to be accomplished by the manufacture of in vitro isolated CIA aneurysm half vessel and full vessel models, the analysis of continuous water flow through those models, and comparison of the empirical observation of dye movement to the predictions of a COMSOL simulation. The use of water, which has a lower viscosity than blood (1.0 cP compared to about 3.0 to $4.0 \mathrm{cP}$ ), and continuous flow conditions prevent this study from translating directly to conclusions about in vivo flow conditions. Rather, it establishes likely average velocity and shear stress values that would be observed in physiological conditions. Introducing pulsatility in particular may demonstrate unsteadiness to the jet, and vortex within the aneurysm, and it would establish the ranges of velocity and shear stress values which would not be obtained from studies assuming continuous flow. The results of the half vessel study indicated poor agreement between measured maximum velocity values and those predicted by the COMSOL simulation; with 95\% confidence, a one sample, single tailed t-test demonstrated a significant difference between the measured and predicted velocity plots. Although the empirical trials followed a similar trend of velocity decreasing with 
distance traveled in the aneurysm model. Improved lighting conditions and improved frame rates for capturing dye may allow the gap between the empirical data and the COMSOL simulation to be closed. However, the subjectivity involved in selecting a dye front location in a dye plume may prove to be an insurmountable problem for flow visualization studies that rely on dye tracking, even with improved lighting and frame rates during image capture.

\subsection{Conclusions and Future Work}

As far as the literature review indicated, the dye imaging trials were a novel, and cost effective way to assess fluid velocity profiles in channels on the order of centimeters in diameter. This method of assessing fluid velocities proved advantageous in the imaging of larger channels because it did not require the use of a microscope with a limited focal length to capture fluid motion. Moreover, the food dye used in this project was less expensive than fluorescent beads typically used in PIV, making it the most cost effective method of tracking fluid flow in the large channel analyzed in this project.

The manufacturing techniques used for the full vessel model could be improved upon by either improving the dissolution rate of the aneurysm model set in PDMS, or by joining two half vessel models after a plasma treatment. Additionally, imaging fluid flow in the full vessel model would present unique challenges, such as the need for a fluid matching the refractive index of PDMS, and it would introduce the need to orient the construct with the outlets on top to allow the evacuation of air bubbles without allowing fluid to run down the sides of the model to avoid optical distortion in the imaging process.

The experiments conducted here did not demonstrate that dye tracking was an effective means of assessing fluid velocities in a large channel, although improvements to flow visualization techniques for larger channels could likely be made based on the 
results of this study. Future projects could build on this work by improving control over lighting conditions, use a camera for image capture that offers a higher frame rate to increase the accuracy of velocity measurements, and explore possible PIV techniques such as tracking metal shavings to remove the subjectivity involved in selecting a dye front from a plume. Additionally, it would be advisable for future projects to take steps to improve the fidelity of the model manufacturing process to the model used in computer simulations, and seek greater agreement between the boundary conditions of the computer simulation and the in vitro model to ensure greater agreement between empirical measurements and the predictions of FEA. Such improvements would cause future projects to place more emphasis on and commit more resources to the design of a fixture for image collection to ensure consistent and reliable results. Future work capturing videos of dye motion at higher frame rates would necessarily produce a larger volume of data. Therefore, it would be worthwhile to investigate automation techniques for tasks such as frame extraction from videos, image processing, and the computations which were completed in Excel to process the data.

What has been made clear by this study is that several separate steps to this flow visualization project contain unresolved technical challenges which deserve closer attention in future work. Therefore, dividing up the tasks undertaken here into separate projects would allow closer attention to each of these problems. One possible division of projects is as follows:

1. Construction and use of a fixture for flow visualization of particles through a channel of simple geometry that features laminar or inviscid flow. This project would face challenges such as controlling lighting conditions, translating PIV to non-microfluidic channels, removing air bubbles and other factors which may affect flow or lead to optical distortion, and determining which frame rate would be most appropriate for flow visualization. 
2. Development of techniques to automate data processing. Visualizing flow at higher framerates and over the course of a larger number of experiments would result in a volume of data which may be prohibitively time consuming to manually input to a spread sheet. A script which could process images, extract particle locations and displacements between frames, and perform the necessary computations to characterize the velocity profile would be desirable.

3. Exploration of methods of manufacturing full vessel models possibly based on empirical models from an angiogram. A project which accomplishes this by selectively dissolving away the model polymer may require the use of small scale experiments to determine which factors have the greatest influence on the dissolution rate of PVA, such as water temperature, skin thickness, infill percentage, and exposed surface area. 


\section{BIBLIOGRAPHY}

[1] R. S. Sandhu and I. I. Pipinos, "Isolated iliac artery aneurysms," Seminars in Vascular Pathology, vol. 18, no. 4, pp. 209-15, 2005.

[2] M. T. Dix, F. and H. Al-Khaffaf, "The isolated internal iliac artery aneurysm-a review," European Journal of Vascular and Endovascular Surgery, vol. 30, no. 2, pp. 119-29, 2005.

[3] Columbia Department of Surgery, “Aortic surgery program.” Online.

[4] S. M. Santilli, S. M. Wernsing, and E. S. Lee, "Expansion rates and outcomes for iliac artery aneurysms," Journal of Vascular Surgery, vol. 31, pp. 114-21, January 2000.

[5] A. J. Dean and B. S. Ku., "Abdominal aortic aneurysm.” Online, 2008.

[6] N.a., "About high resolution x-ray ct." Online, 2014.

[7] S. Berger, W. Goldsmith, and E. Lewis, Introduction to Bioengineering. Oxford University

Press.

[8] M. Armon, P. Wenham, S. Whitaker, R. Gregson, and B. Hopkinson, "Common iliac artery aneurysms in patients with abdominal aortic aneurysms," European Journal of Vascular and Endovascular Surgery, vol. 15, no. 3, pp. 255 - 257, 1998.

[9] M. Thiriet, Anatomy and Physiology of the Circulatory and Ventilatory Systems. Springer.

[10] K. Saladin, Anatomy and Physiology. McGraw Hill. 
[11] A. Maradni, S. Emamirazavi, A. Noruzi-javidan, S. H. Mousavi, and A. Khoshnevisan, "Role of matrix metalloproteinases (mmps) and mmp inhibitors on intracranial aneurysms (a review article)," American Journal of Pharmacological Sciences, vol. 1, no. 5, pp. 100-103, 2013.

[12] V. Kumar, Robbins Basic Pathology. Elsevier Saunders and A.K. Abbas and J.C. Aster.

[13] K. A. Freel and W. K. Nutley, "Internal iliac artery aneurysm detected by sonography," Journal of Diagnostic Medical Sonography, vol. 29, no. 5, pp. 234 $-237,2013$.

[14] K. Chiu, V. T. and M. Ahmed, and V. Shrivastava, "Ultrasound measurement for abdominal aortic aneurysm screening: A direct comparison of the three leading methods," European Journal of Vascular and Endovascular Surgery, vol. 48, pp. $231-232$, August 2014.

[15] V. Goyal, S. Sood, and B. Gupta, "Isolated common iliac artery aneurysm: A rare entity," Journal of Clinical and Diagnostic Research, vol. 8, no. 11, pp. ND03 ND04, 2014.

[16] M. Taheri, H. Haghighatkhah, R. Pourghorban, and A. Hosseini, "Multidetector computed tomography findings of abdominal aortic aneurysm and its complications: A pictorial review," Emergency Radiology, vol. 20, no. 5, pp. 443 $-451,2013$.

[17] F. Moll, G. Fraedrich, and F. Verzini, "Management of abdominal aortic aneurysms clinical practice guidelines of the european society for vascular surgery," European Society for Vascular Surgery. 
[18] S. Buckley and C. Buckley, "Endovascular aortoiliac aneurysm repair: Surgical progress through new treatment paradigms and innovative endograft design," AORN Journal.

[19] A. Chemelli, B. Hugl, J. Klocker, M. Thauerer, A. Strasak, W. Jaschke, P. Waldenberger, and I. Chemelli-Steingruber, "Endovascular repair of isolated iliac artery aneurysms," Journal of Endovascular Therapy, vol. 17, pp. $492-503$, August 2010.

[20] C. Duran, J. Naoum, C. Smolock, C. Bavare, M. Patel, J. Anaya-Ayala, A. Lumsden, and M. Davies, "A longitudinal view of improved management strategies and outcomes after iatrogenic iliac artery rupture during endovascular aneurysm repair,” Annals of Vascular Surgery, vol. 27, pp. 1-7, January 2013.

[21] D. Scheinert, M. Schroder, and H. Steinkamp, "Treatment of iliac artery aneurysms by percutaneous implantation of stent grafts," Circulation.

[22] F.A. Lederle, J.A. Freischlag, T.C. Kyriakides, et al., "Outcomes Following Endovascular vs Open Repair of Abdominal Aortic Aneurysm: A Randomized Trial," JAMA, vol. 302, no. 14, pp. 1535-1542, 2009.

[23] M. F. Conrad, R. S. Crawford, J. D. Pedraza, D. C. Brewster, G. M. LaMuraglia, M. Corey, S. Abbara, and R. P. Cambria, "Long-term durability of open abdominal aortic aneurysm repair," Journal of Vascular Surgery, vol. 46, no. 4, pp. $669-675,2007$.

[24] S. Devasenathipathy, J. Santiago, S. Wereley, C. Meinhart, and K. Takehara, "Particle imaging techniques for microfabricated fluidic systems," Experiments in Fluids, vol. 34, no. 4, 2003. 
[25] Merriam-Webster, "Visual dictionary online: Urinary system." Online, 2015.

[26] Pearson Education, "Arterial structure."

[27] M. Burns, Automated fabrication: improving productivity in manufacturing. Prentice Hall.

[28] X. Yan and P. Gu, "A review of rapid prototyping technologies and systems," Computer-Aided Design, vol. 28, no. 4, pp. 307 -318, 1996.

[29] J. McDonald, D. Duffy, J. Anderson, D. Chiu, H. Wu, O. Schueller, and G. Whitesides, "Fabrication of microfluidic systems in poly(dimethylsiloxane)," Electrophoresis, vol. 21.

[30] R. White, "Polydimethylsiloxane (PDMS) on SU-8 Mold: Standard Operating Procedure." Online.

[31] Center of Excellence in Nanoelectronics, "Making flexible molds with pdms." Online.

[32] N. H. Service, "Abdominal aortic aneurysm screening - when it's offered." Online, 2014.

[33] J. R. Cebral, M. A. Castrod, J. E. Burgess, R. S. Pergolizzi, M. J. Sheridanc, and C. M. Putman, "Characterization of cerebral aneurysms for assessing risk of rupture by using patientspecific computational hemodynamics models," American Journal of Neuroradiology.

[34] C. A. Taylor and M. T. Draney, "Experimental and computational methods in cardiovascular fluid mechanics," Fluid Mechanics, vol. 36. 
[35] M. Castro, C. Putman, and J. Cebral, "Computational fluid dynamics modeling of intracranial aneurysms: Effects of parent artery segmentation on intra-aneurysmal hemodynamics," American Journal of Neuroradiology.

[36] S. Tateshima, K. Tanishita, H. Omura, J. Villablanca, and F. Vinuela, "Intraaneurysmal hemodynamics during the growth of an unruptured aneurysm: In vitro study using longitudinal ct angiogram database," American Journal of Neuroradiology.

[37] B. J. Kirby, Micro- and Nanoscale Fluid Mechanics: Transport in Microfluidic Devices. Cambridge University Press.

[38] R. L. Fournier, Basic Transport Phenomena in Biomedical Engineering. CRC Press.

[39] W. R. Debler, Fluid Mechanics Fundamentals. Prentice Hall.

[40] F. Ene, C. Gachon, P. Delassus, R. Carroll, F. Stefanov, P. O’Flynn, and L. Morris, "In vitro evaluation of the effects of intraluminal thrombus on abdominal aortic aneurysm wall dynamics," Medical Engineering \& Physics, vol. 33, no. 8, pp. $957-966,2011$.

[41] J. C. Lasheras, "The biomechanics of arterial aneurysms," Annual Review of Fluid Mechanics, vol. 39.

[42] A. M. Malek, S. L. Alper, and S. Izumo, "Hemodynamic shear stress and its role in atherosclerosis," Journal of the American Medical Association, vol. 282, pp. 2035 - 2042, December 1999.

[43] H. Mayer, "Less-traditional microfabrication techniques: Soft lithography and beyond, \& microfluidics.” Online, March 2015. 
[44] American Heart Association, "Images and case reports in interventional cardiology." Online,

2015.

[45] J. J. Stickel and R. L. Powell, "Fluid mechanics and rheology of dense suspensions," Annual

Review of Fluid Mechanics, vol. 37.

[46] A. J. Smits, "A Physical Introduction to Fluid Mechanics" John Wiley \& Sons, Inc.

[47] R. W. Fox, A. T. McDonald, and P. J. Pritchard, Introduction to Fluid Mechanics. Wiley. 


\section{A. COMPUTATIONS}

Volumetric flow rate of pump was computed based on time required to fill $1 \mathrm{~L}$ beaker.

$$
\frac{1 L}{26.57 \mathrm{sec}} * \frac{60 \mathrm{sec}}{\min }=2.258 \mathrm{~L} / \mathrm{min}
$$

The volumetric flow rate from the pump was used to compute the velocity at the inlet of the half-vessel model in the COMSOL simulation.

$$
\frac{(0.5 * 0.02 \mathrm{~m})^{2} * \pi}{2} * \frac{2.258 L}{\min } * \frac{1 \mathrm{~m}^{3}}{1000 \mathrm{~L}} * \frac{60 \mathrm{sec}}{\min }=0.2397 \mathrm{~m} / \mathrm{s}
$$

A Reynolds Number through half-vessel aorta was calculated to aid in choice of flow regime (e.g. Stokes, Laminar, Inviscid) in COMSOL.

$$
R e=\frac{\rho v L}{\mu}=\frac{1000 \mathrm{~kg} / \mathrm{m}^{3} * 0.2397 \mathrm{~m} / \mathrm{s} * 4 * \frac{1.5708 \times 10^{-4} \mathrm{~m}^{2}}{\pi * 0.01 \mathrm{~m}+0.02 \mathrm{~m}}}{1.0 \times 10^{-3} \mathrm{~Pa} * \mathrm{~s}}=3172
$$




\section{B. ANGIOGRAM DIMENSIONS}

Table 5: Arterial Diameter Measurements Extracted from Angiogram.

\begin{tabular}{|c|c|c|c|}
\hline Blood Vessel & Distance (pixels) & Distance (mm) & Mean Dist $(\mathrm{mm})$ \\
\hline \multirow[t]{3}{*}{ Aorta } & 82.54 & 20.51 & $20.00 *$ \\
\hline & 79.36 & 19.69 & \\
\hline & 79.71 & 19.80 & \\
\hline \multirow[t]{3}{*}{ CIA } & 61.13 & 15.19 & 13.69 \\
\hline & 52.01 & 12.92 & \\
\hline & 52.15 & 12.96 & \\
\hline \multirow[t]{3}{*}{ IIA } & 34.79 & 8.64 & 9.04 \\
\hline & 36.72 & 9.12 & \\
\hline & 37.64 & 9.35 & \\
\hline \multirow[t]{3}{*}{ EIA } & 34.02 & 8.45 & 9.27 \\
\hline & 34.79 & 8.64 & \\
\hline & 43.08 & 10.70 & \\
\hline
\end{tabular}

*Assumed value 


\section{ANEURYSM MODEL DESIGN}

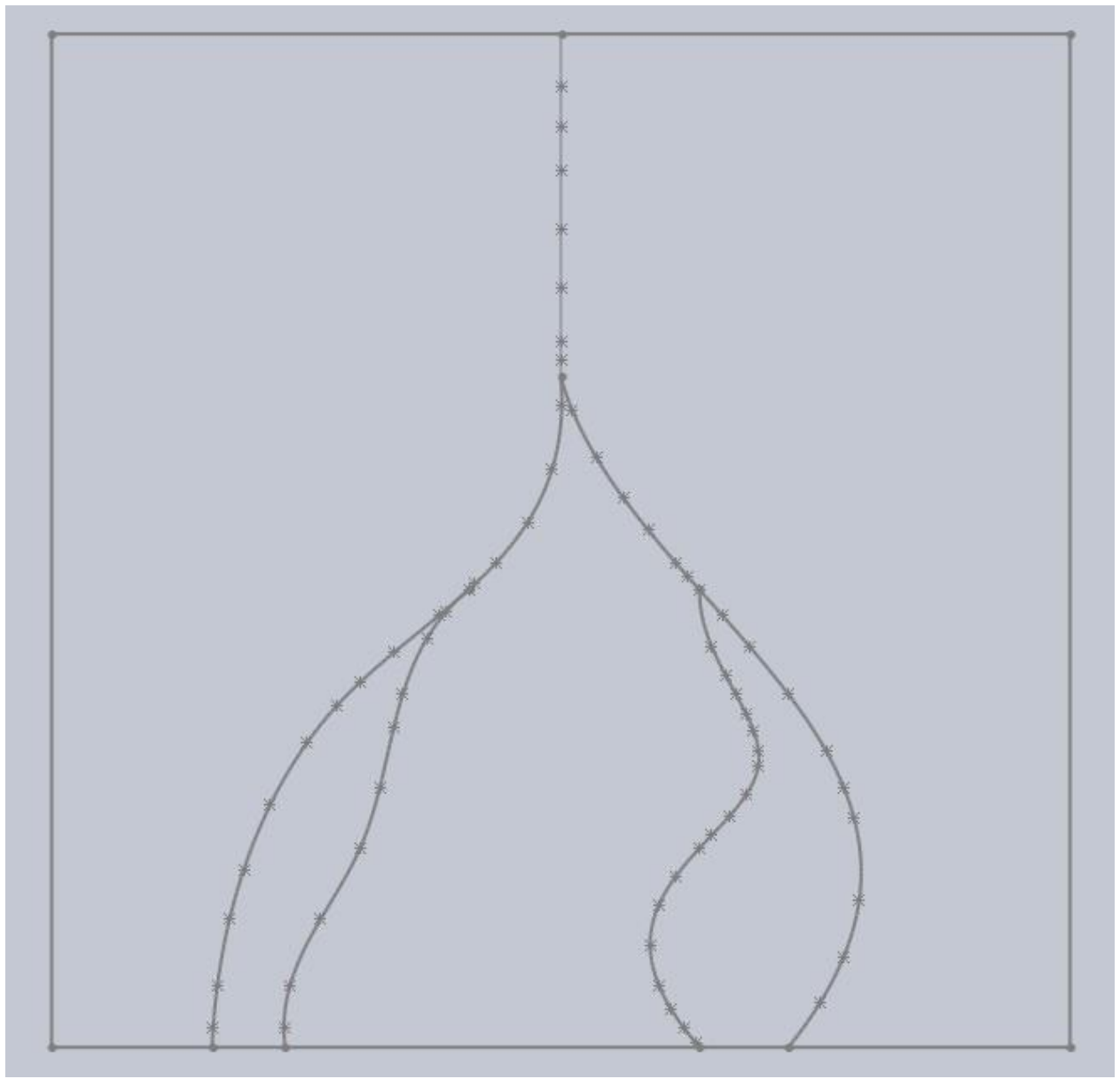

Figure 28: The aneurysm model was constructed from a series of splines with points placed at semi-regular intervals along the splines. 


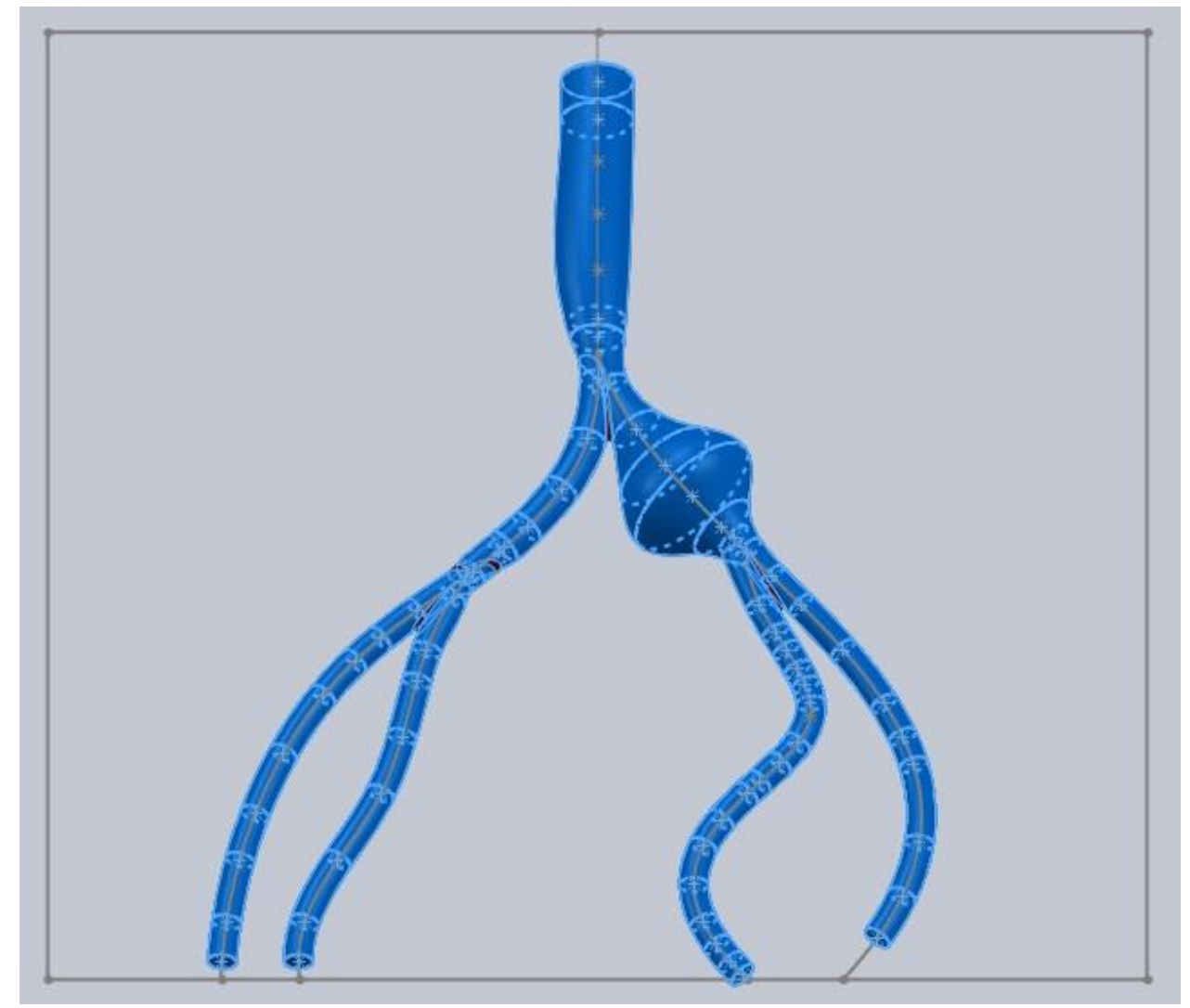

Figure 29: Circles at desired dimensions were centered on the chosen points, perpendicular to the spline. Next, a loft was applied to connect the circles and obtain the organic form of vasculature. 


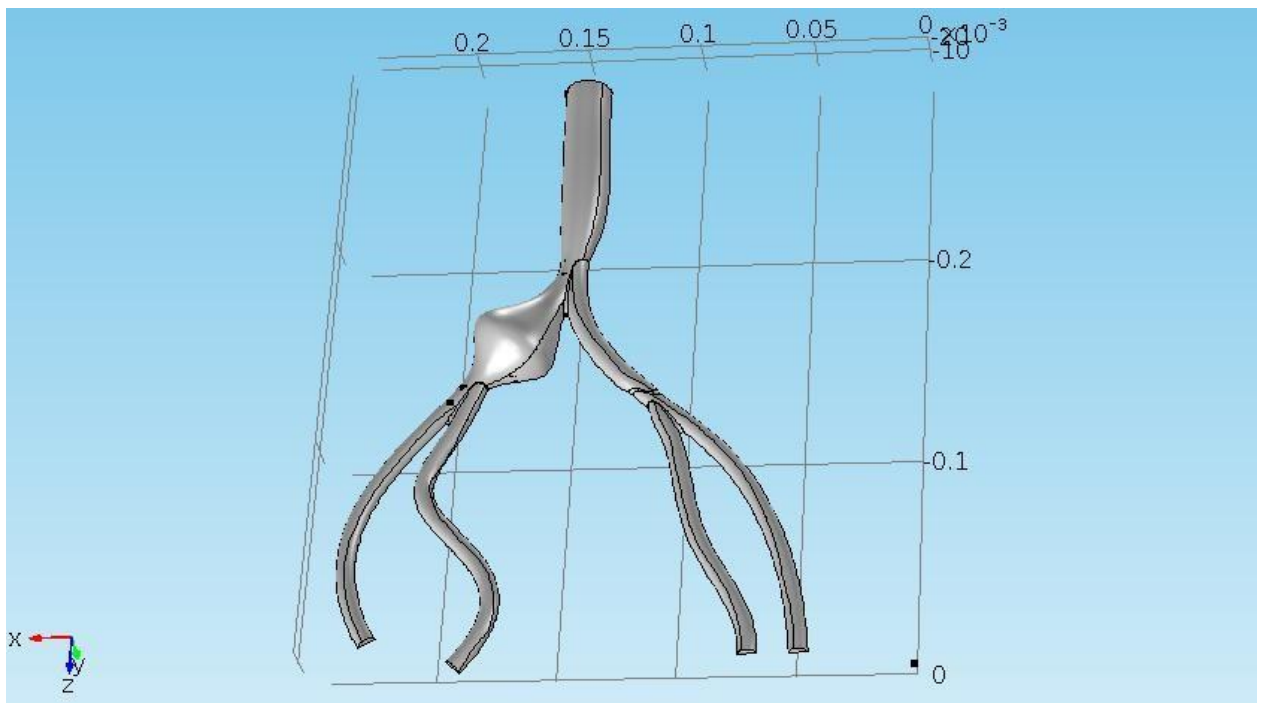

Figure 30: The geometry was exported to COMSOL, cut in half along the $\mathrm{x}-\mathrm{z}$ plane. The COMSOL simulation did not include the connector used in the dye tracking study, assuming a uniform flow field at the inlet, leading to a discrepancy in the boundary conditions.

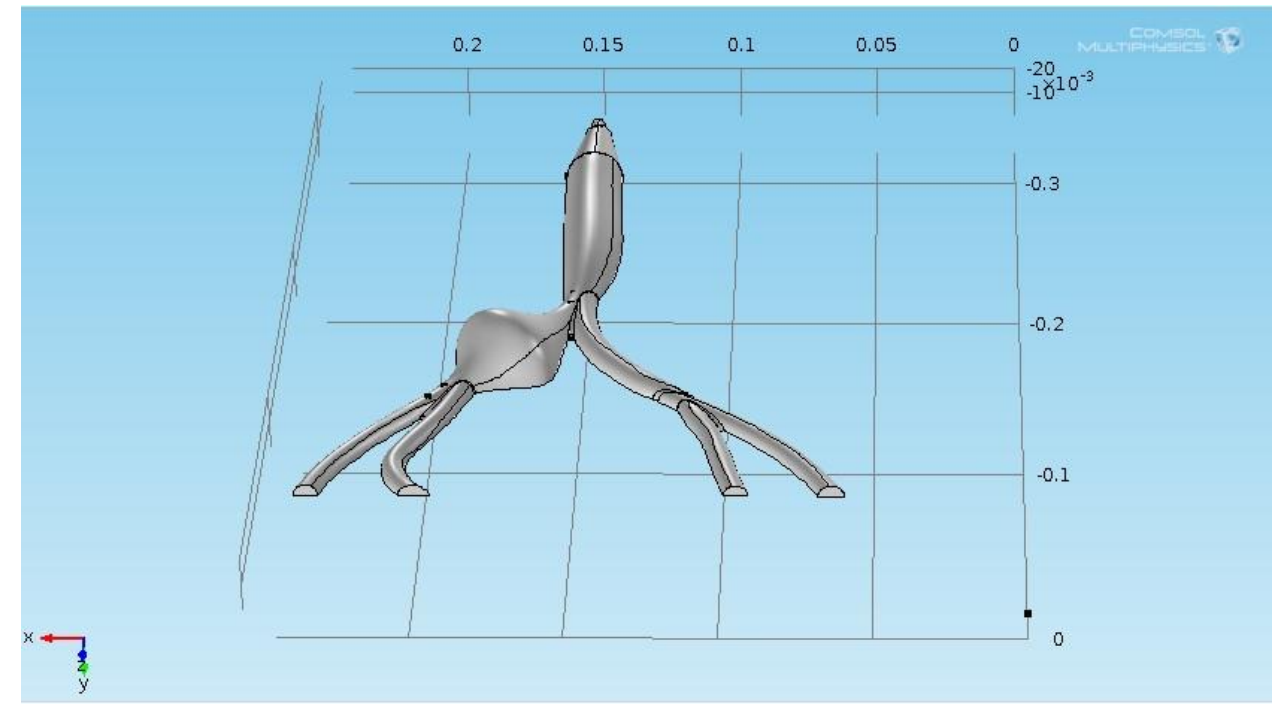

Figure 31: In preparation for FDM manufacturing, a $3 \mathrm{~cm}$ conical connector was added to the end of the half vessel model, and the EIA's and IIA's were trimmed to save space and material. 


\section{FRAMES OF HALF VESSEL IMAGING TRIAL AFTER THRESHOLD}

Six imaging trials (or Runs) were conducted over the course of the experiment. Each frame used in this study is listed according to it's run number and frame number.

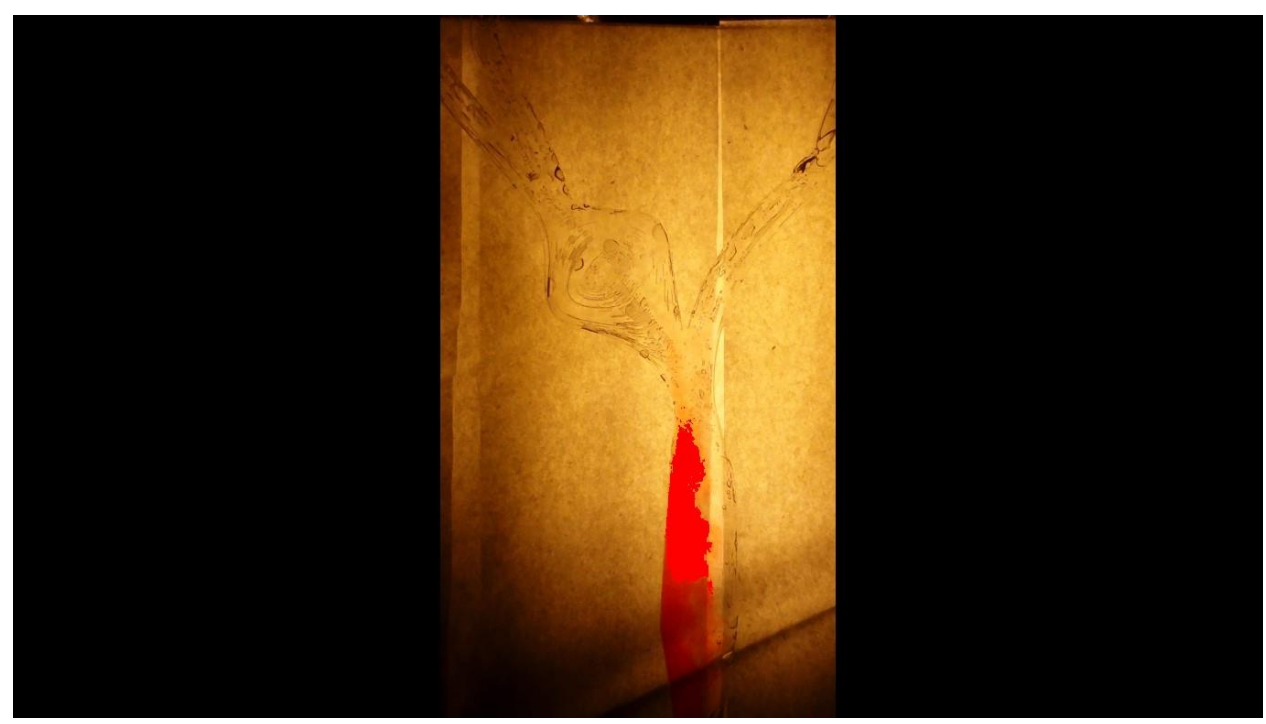

Figure 32: Run 1, Frame 1.

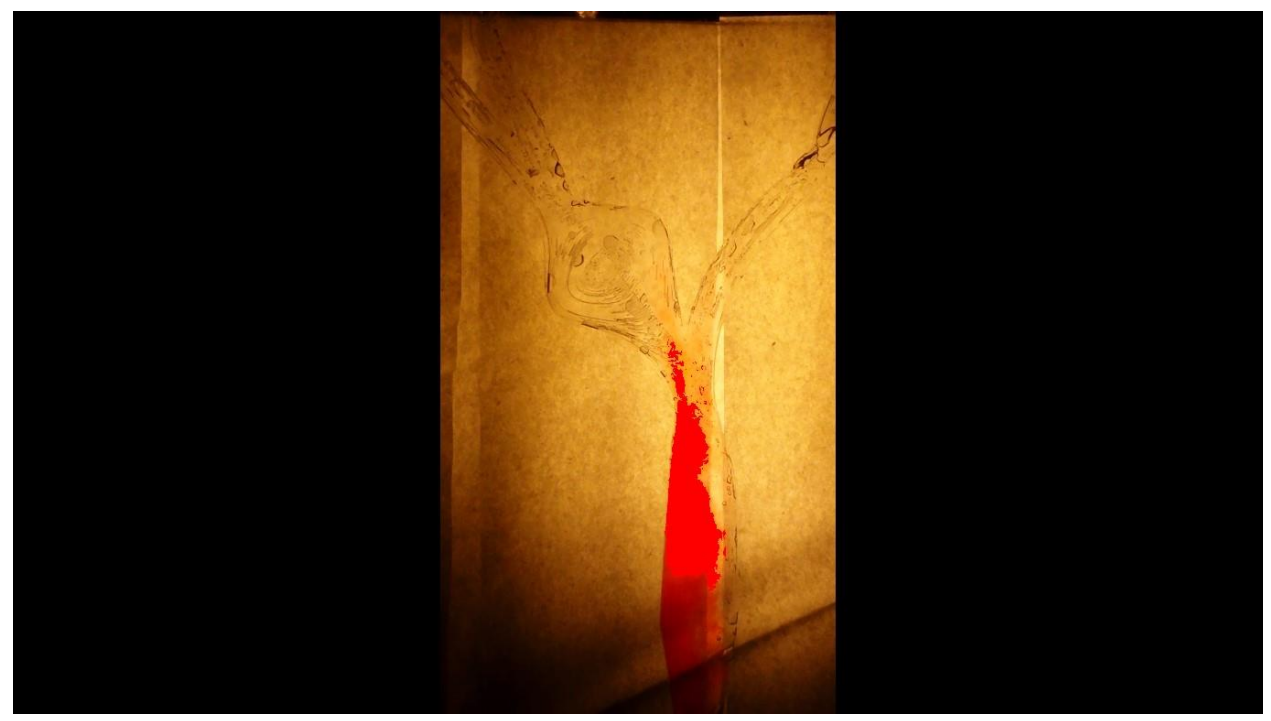

Figure 33: Run 1, Frame 2. 


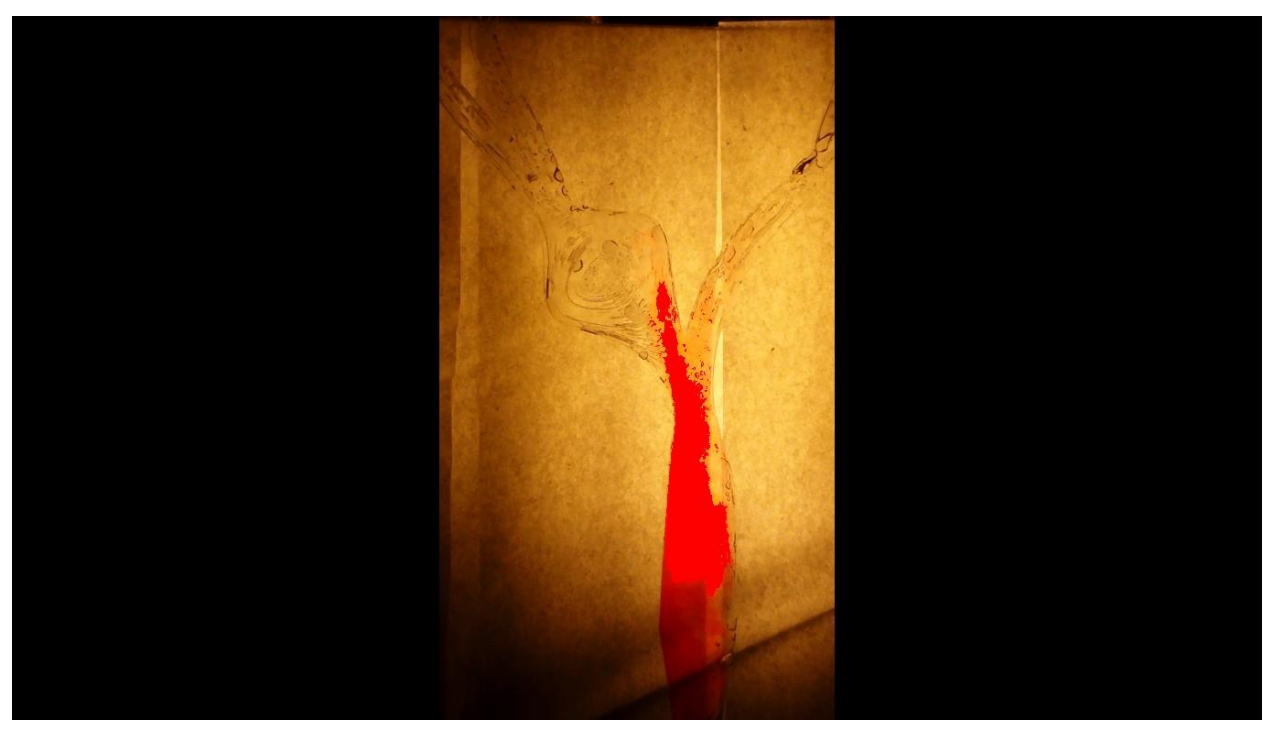

Figure 34: Run 1, Frame 3.

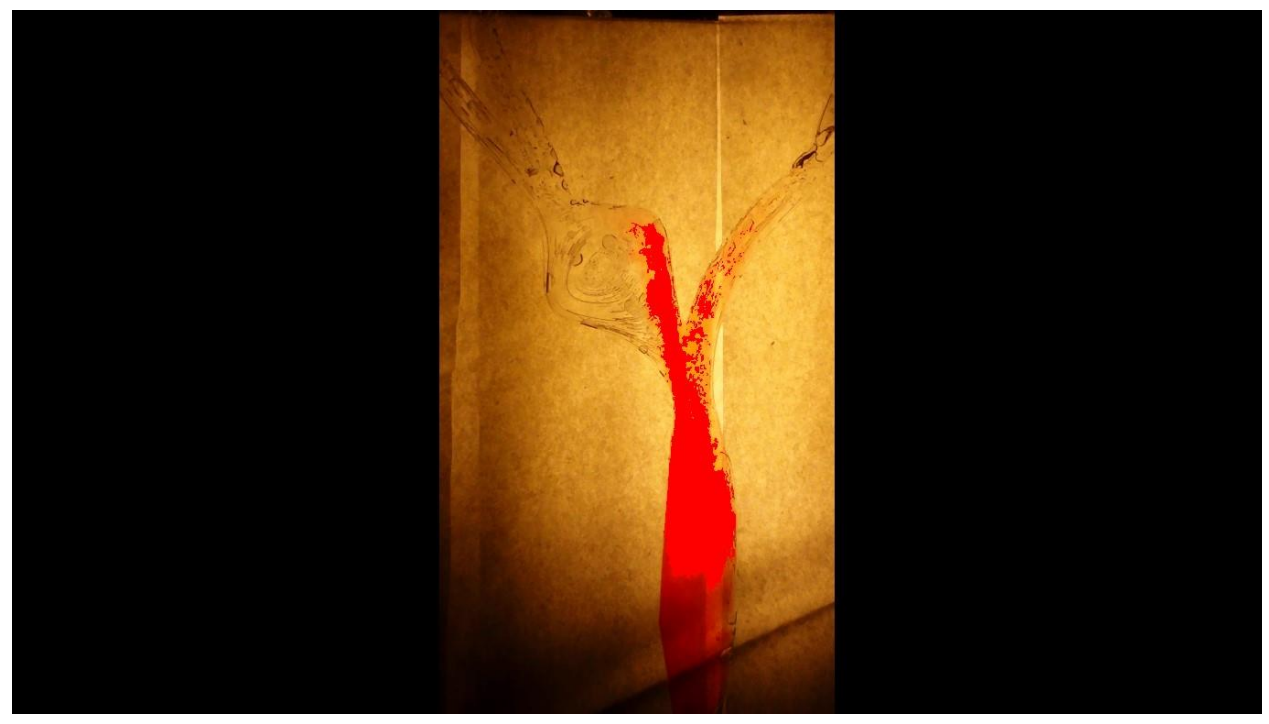

Figure 35: Run 1, Frame 4. 


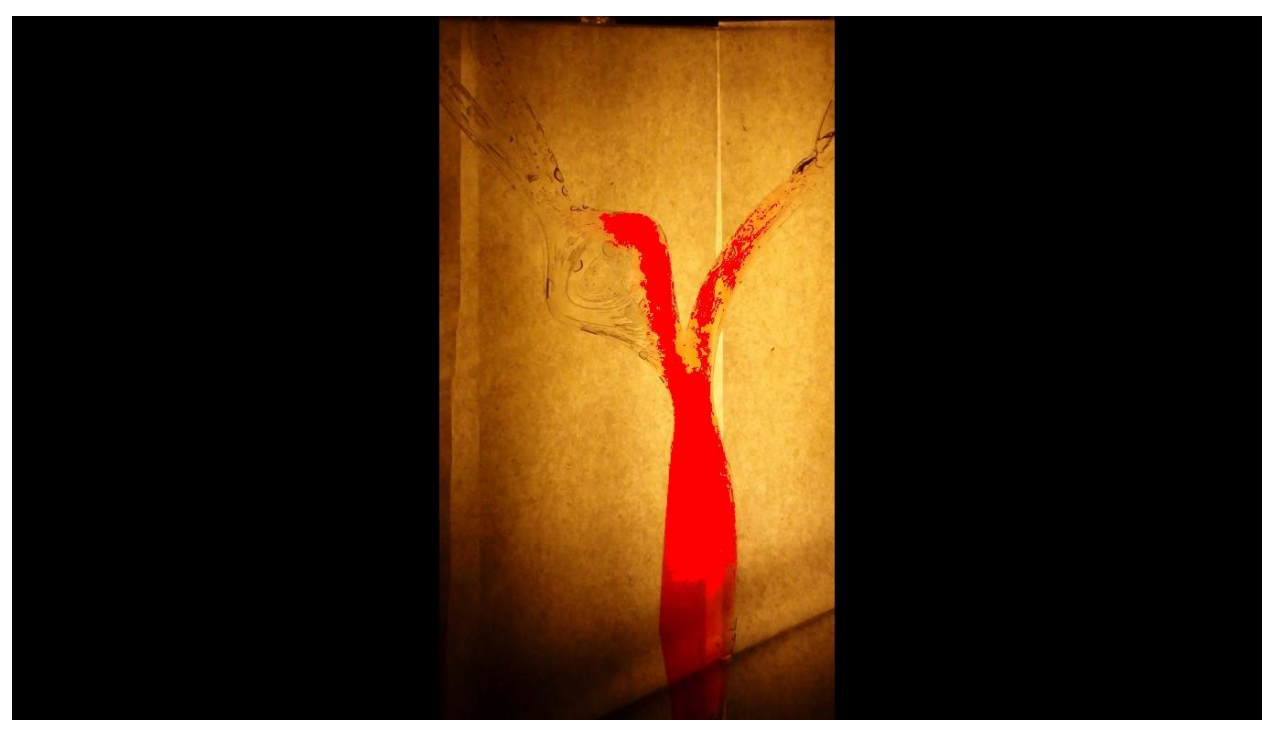

Figure 36: Run 1, Frame 5.

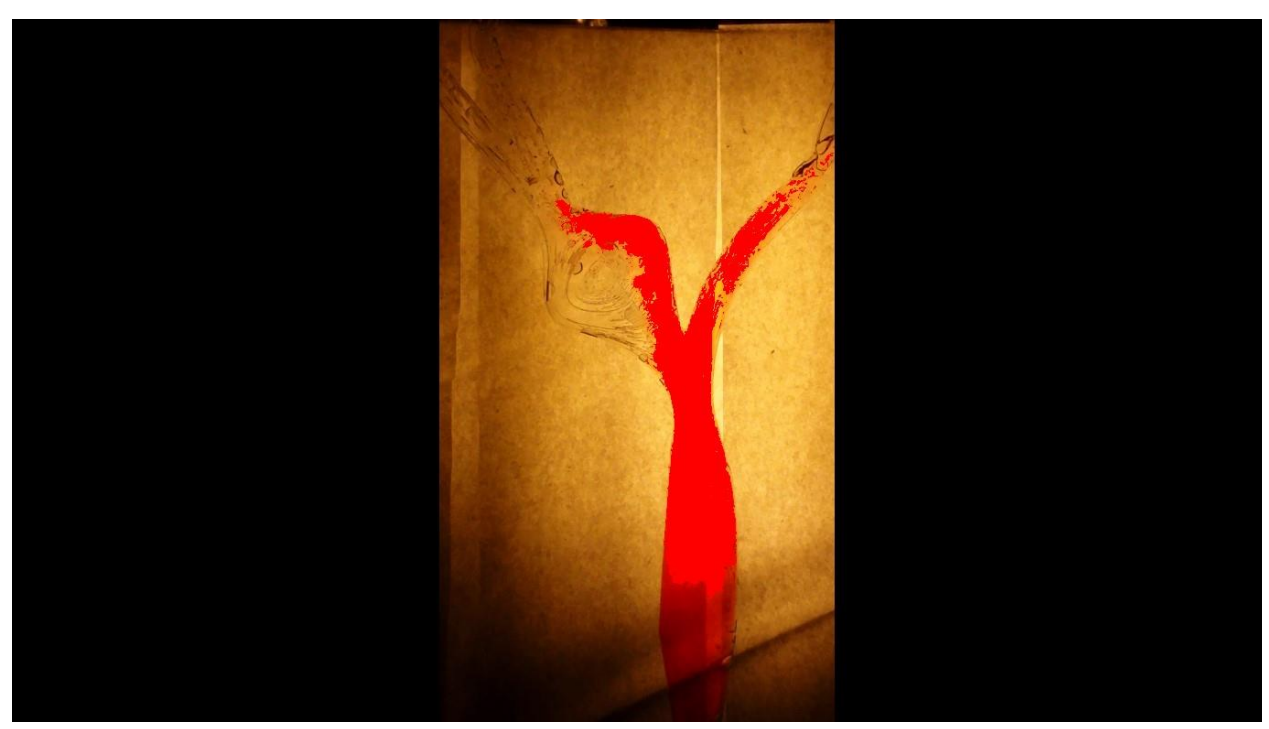

Figure 37: Run 1, Frame 6. 


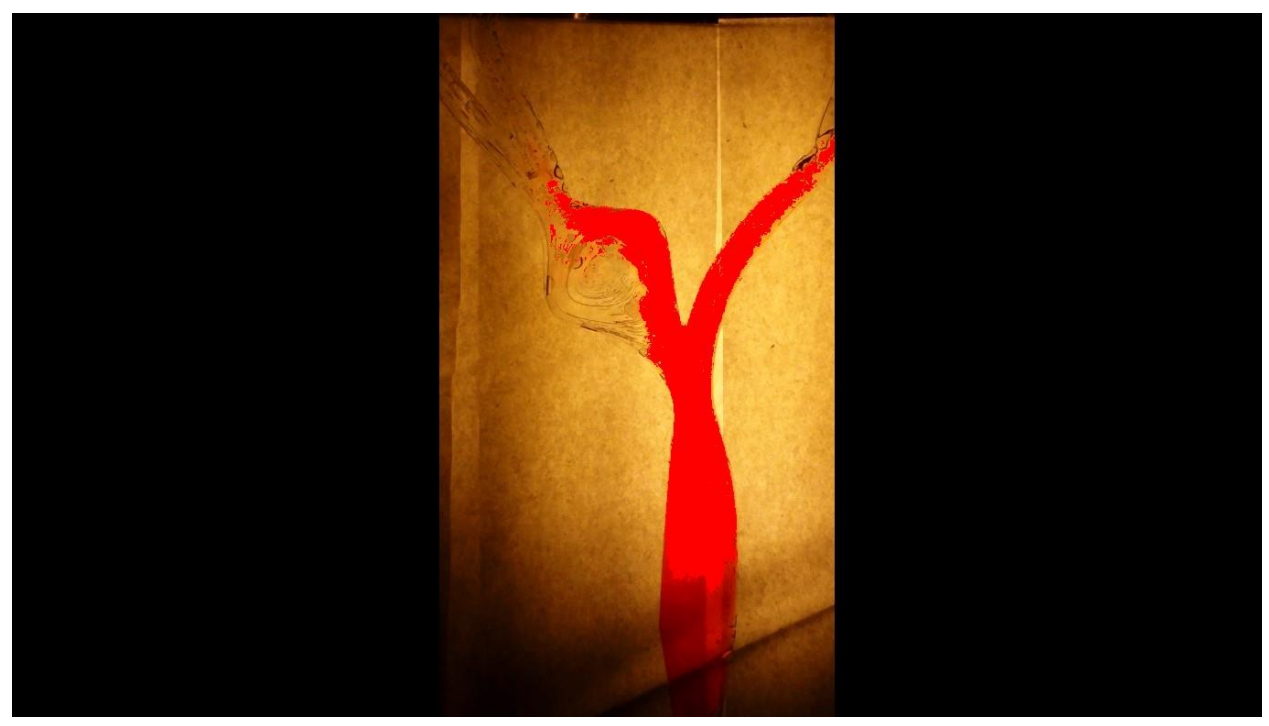

Figure 38: Run 1, Frame 7.

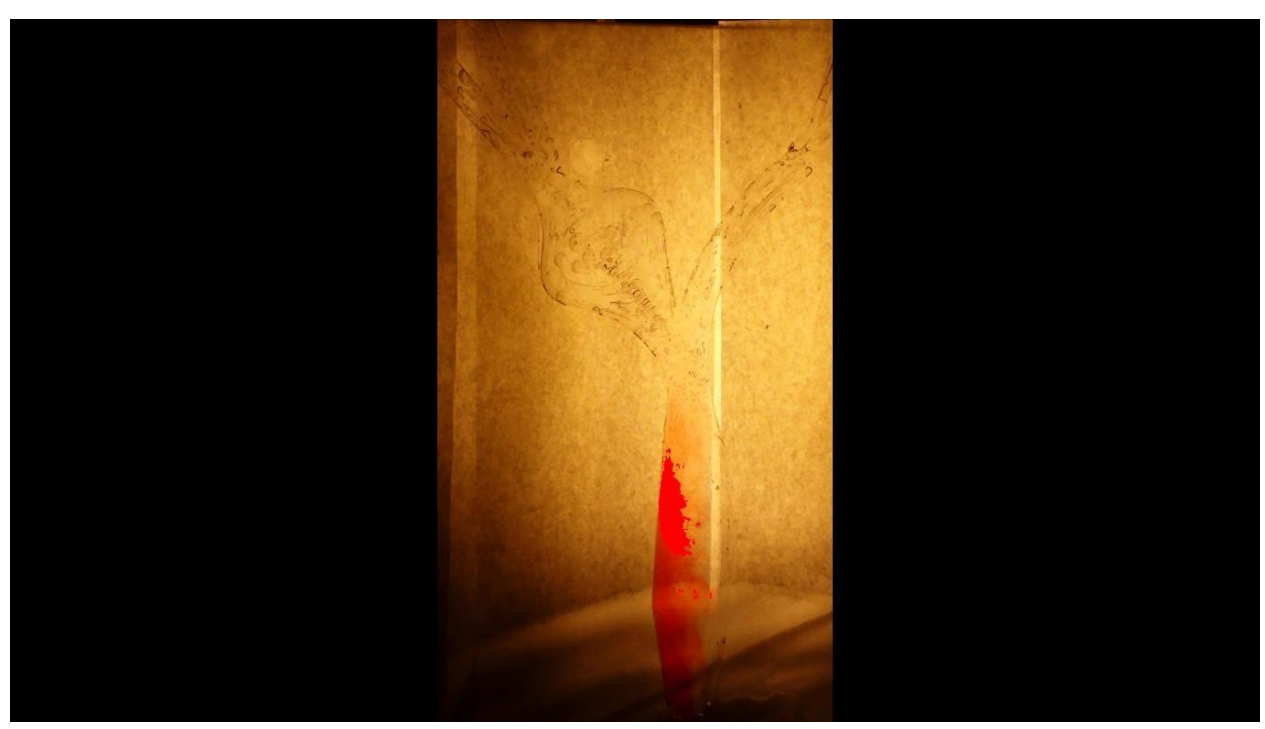

Figure 39: Run 2, Frame 1. 


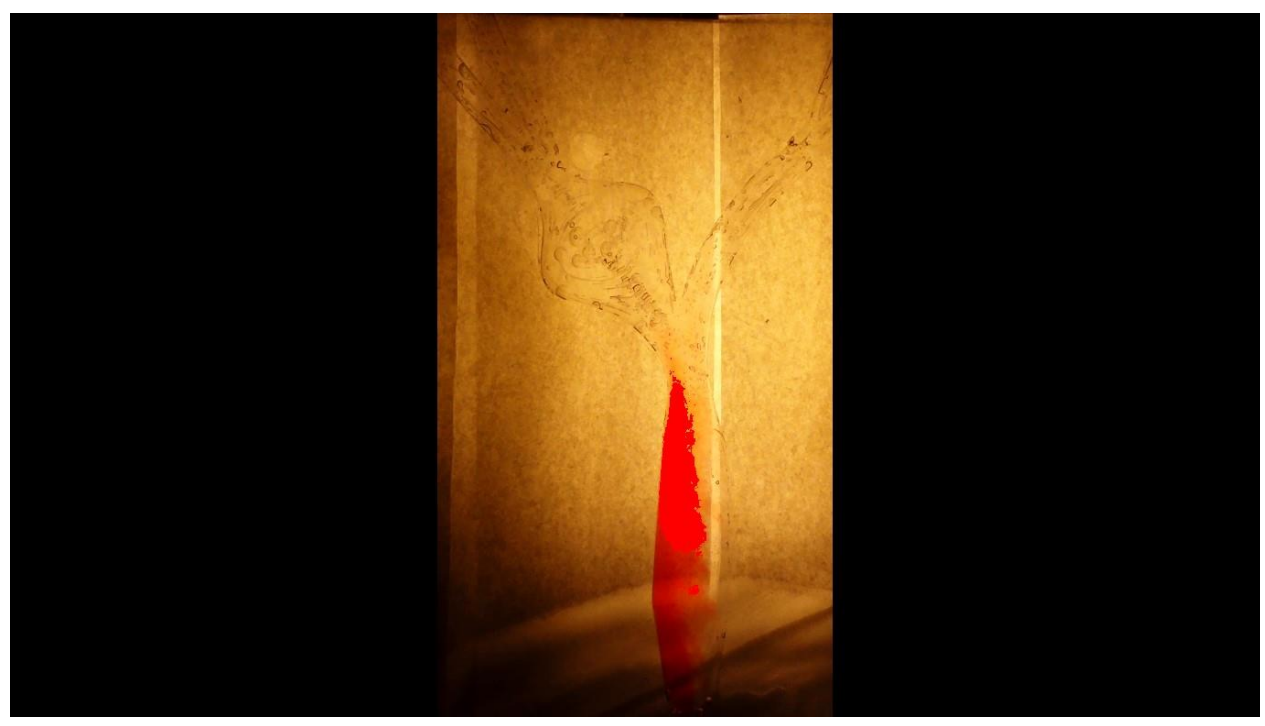

Figure 40: Run 2, Frame 2.

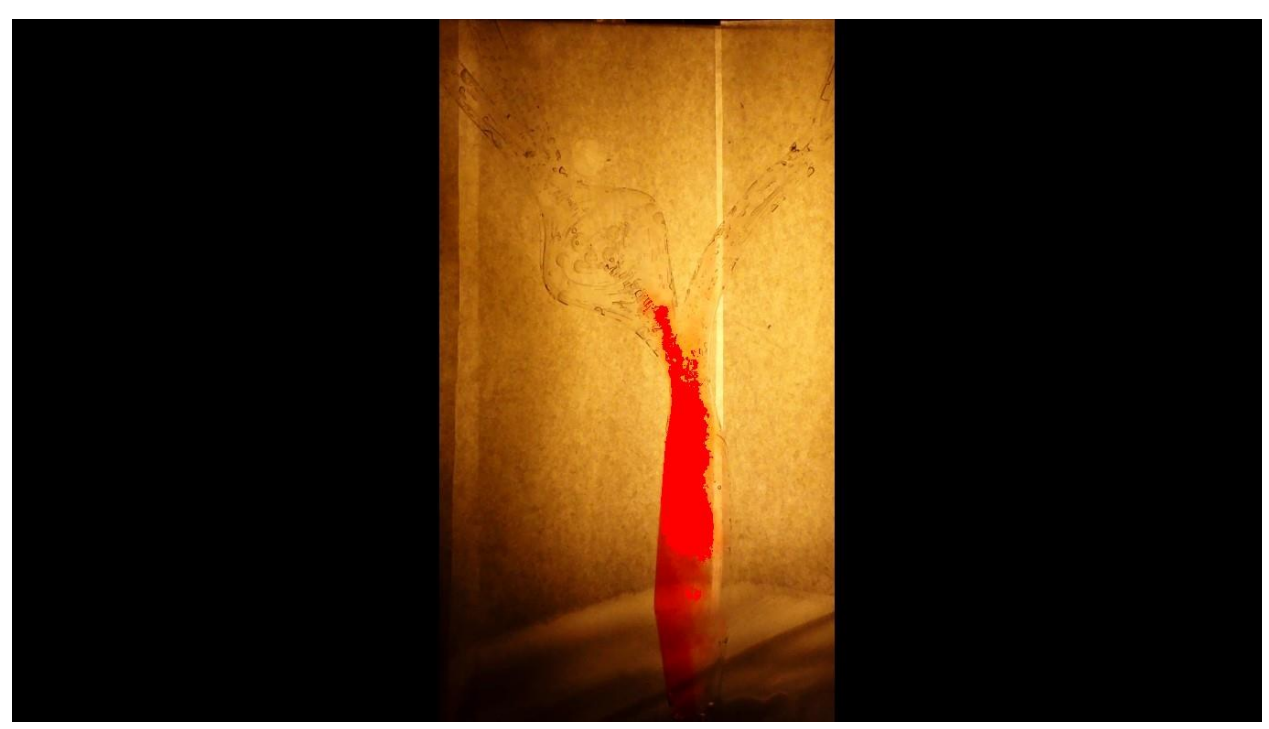

Figure 41: Run 2, Frame 3. 


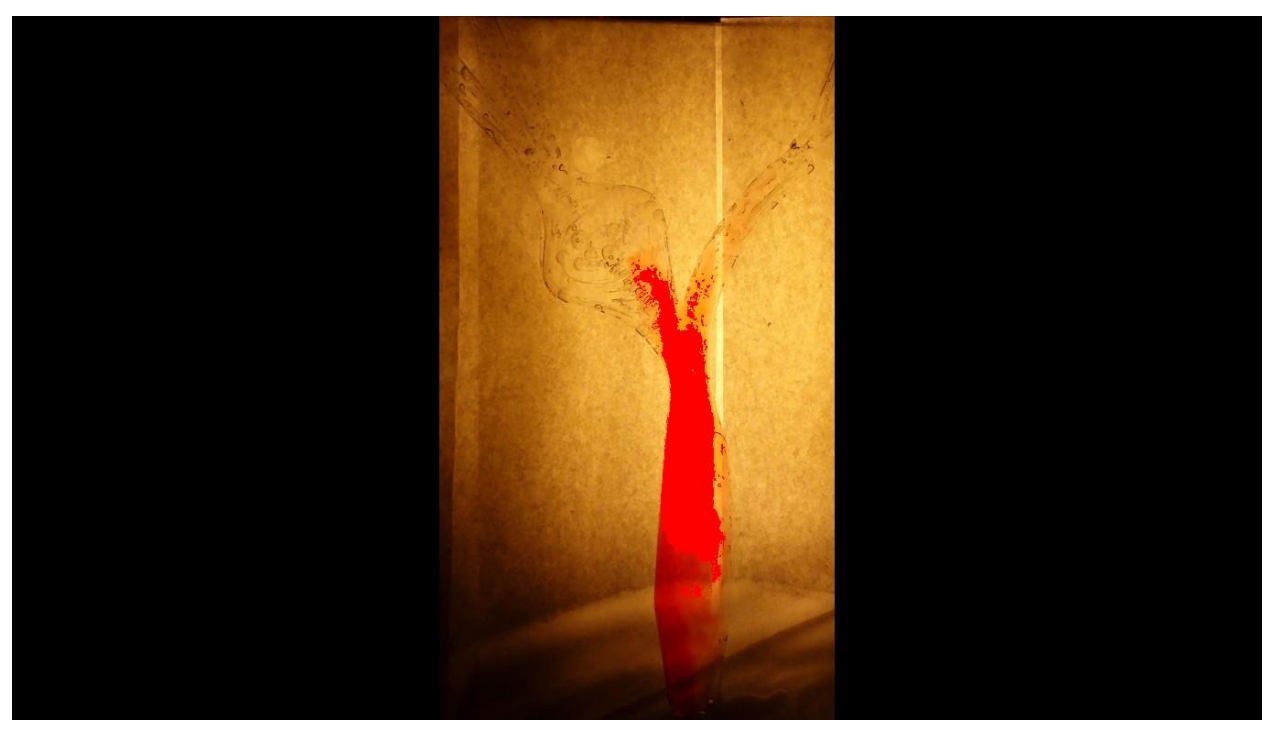

Figure 42: Run 2, Frame 4.

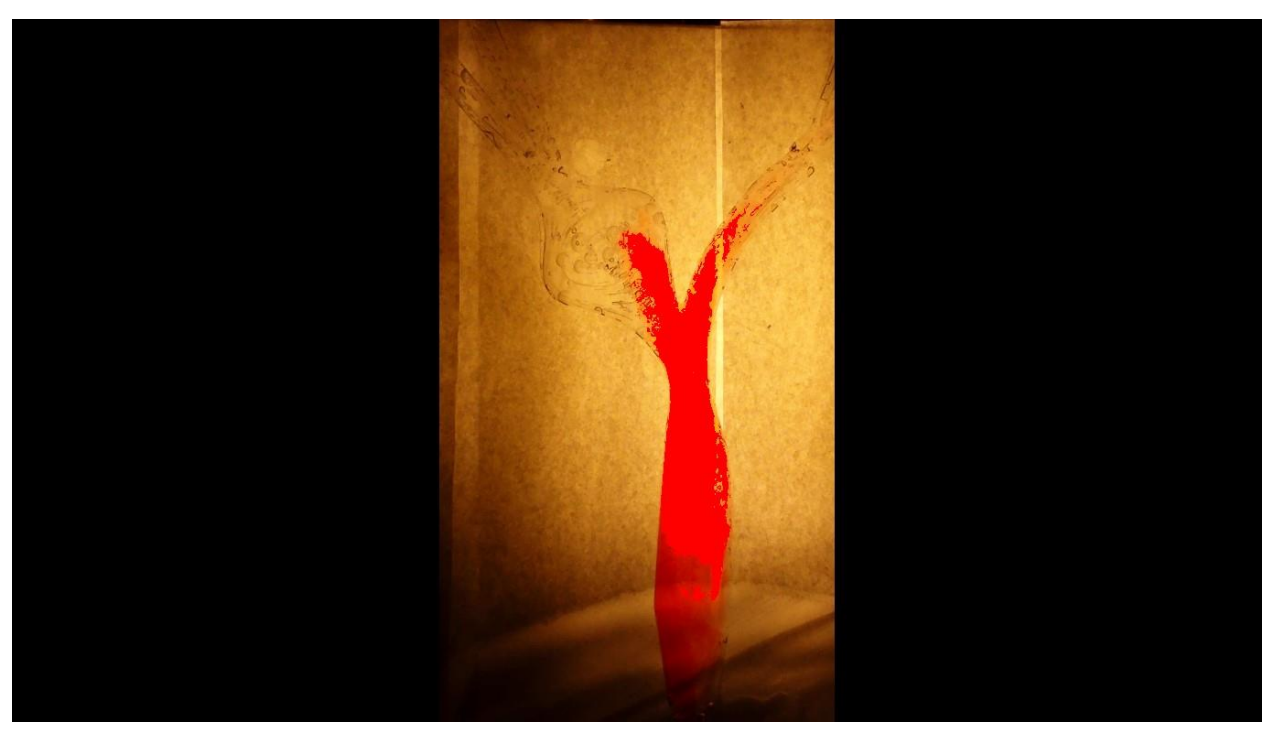

Figure 43: Run 2, Frame 5. 


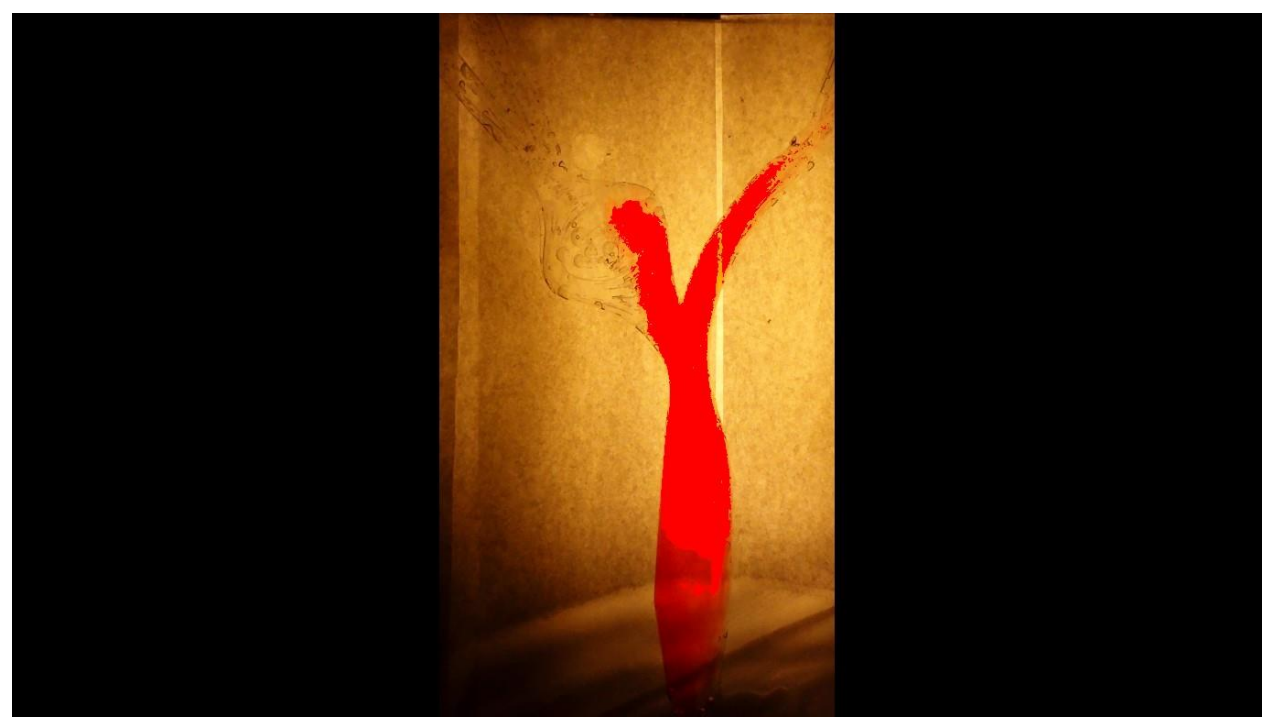

Figure 44: Run 2, Frame 6.

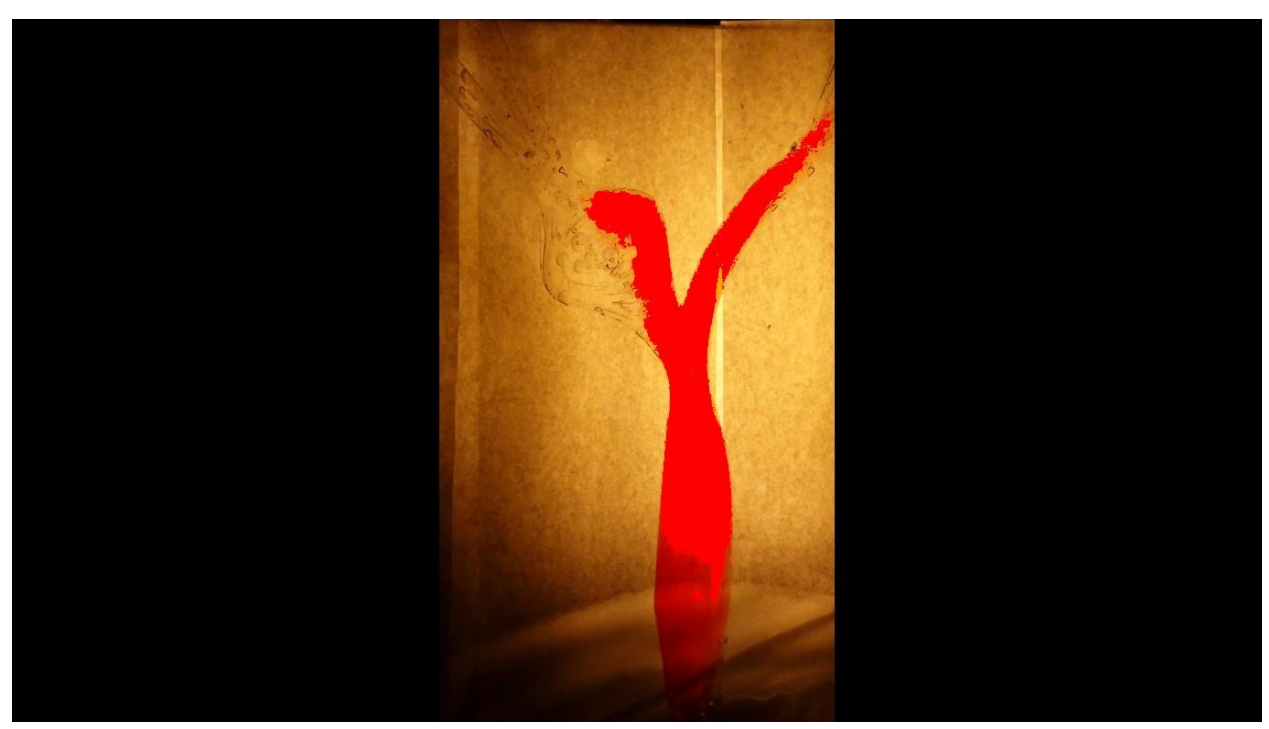

Figure 45: Run 2, Frame 7. 


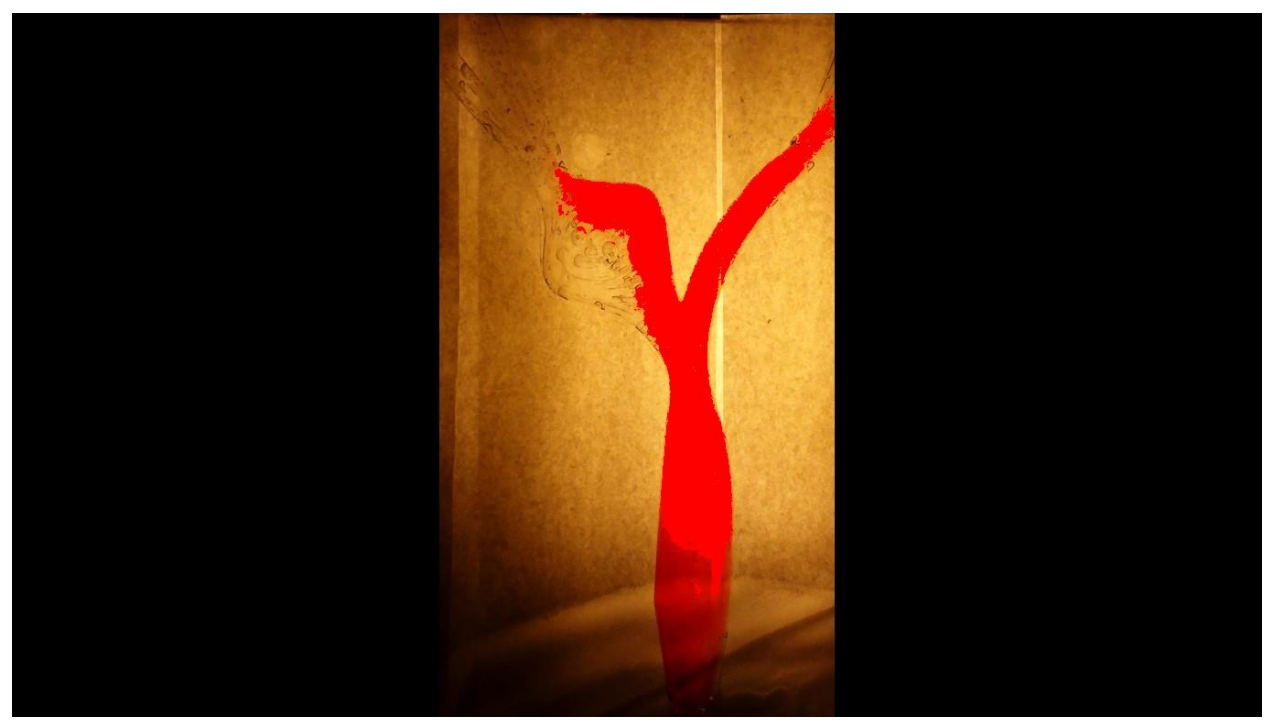

Figure 46: Run 2, Frame 8.

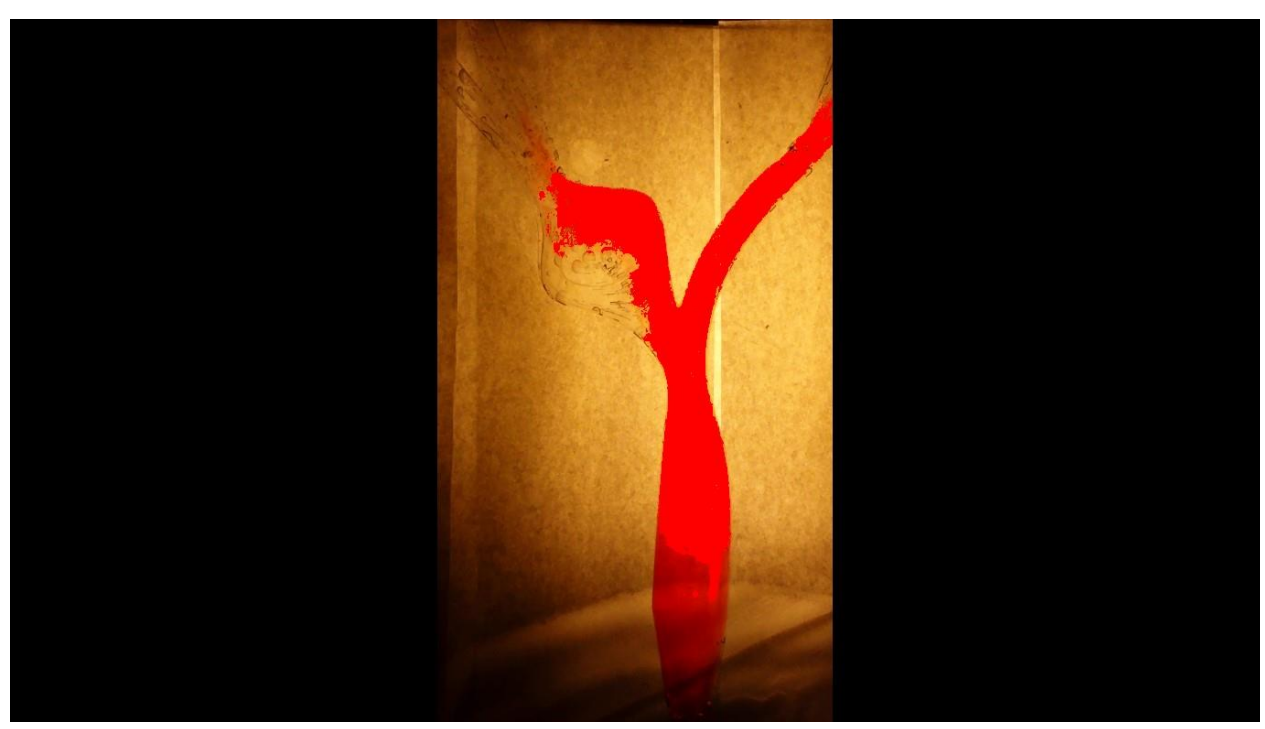

Figure 47: Run 2, Frame 9. 


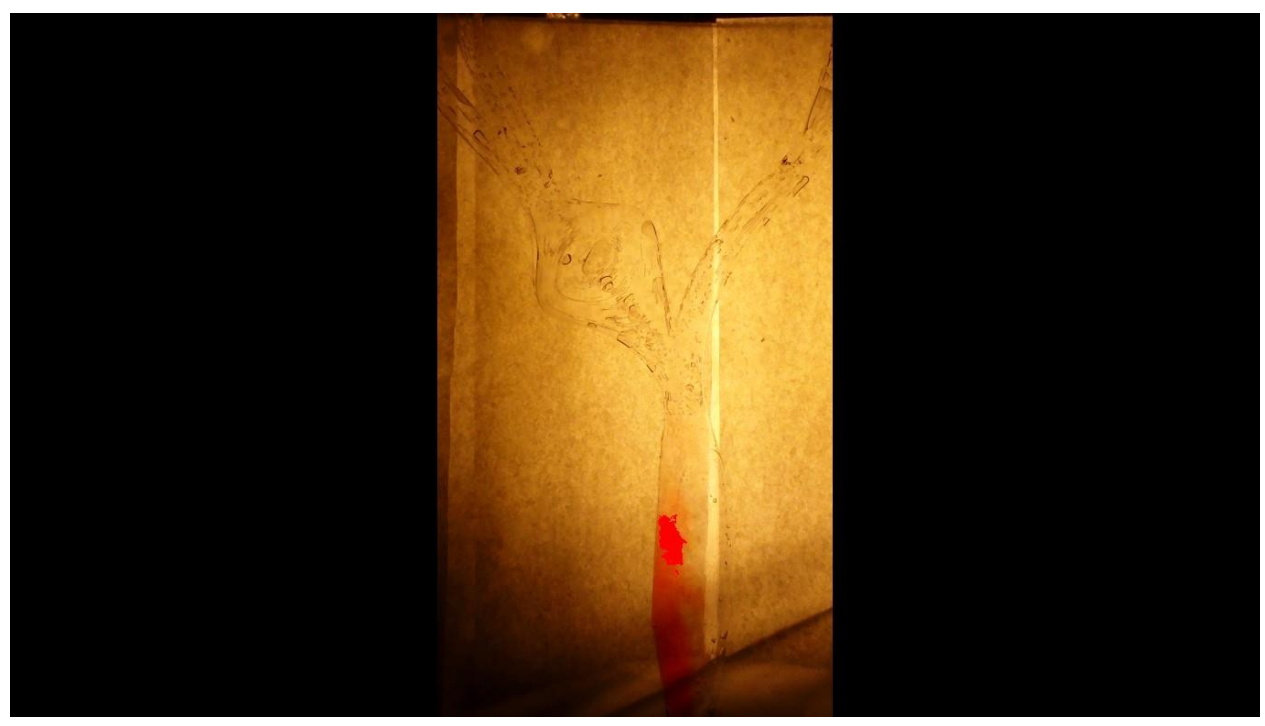

Figure 48: Run 3, Frame 1.

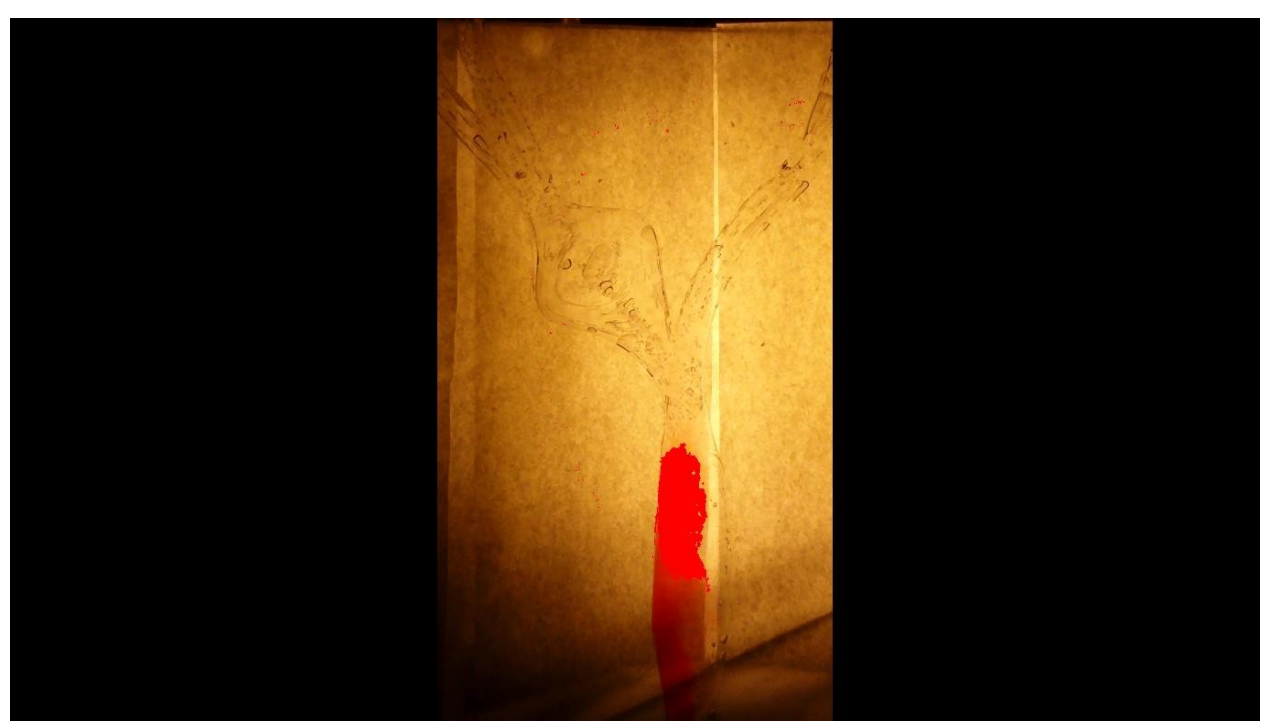

Figure 49: Run 3, Frame 2. 


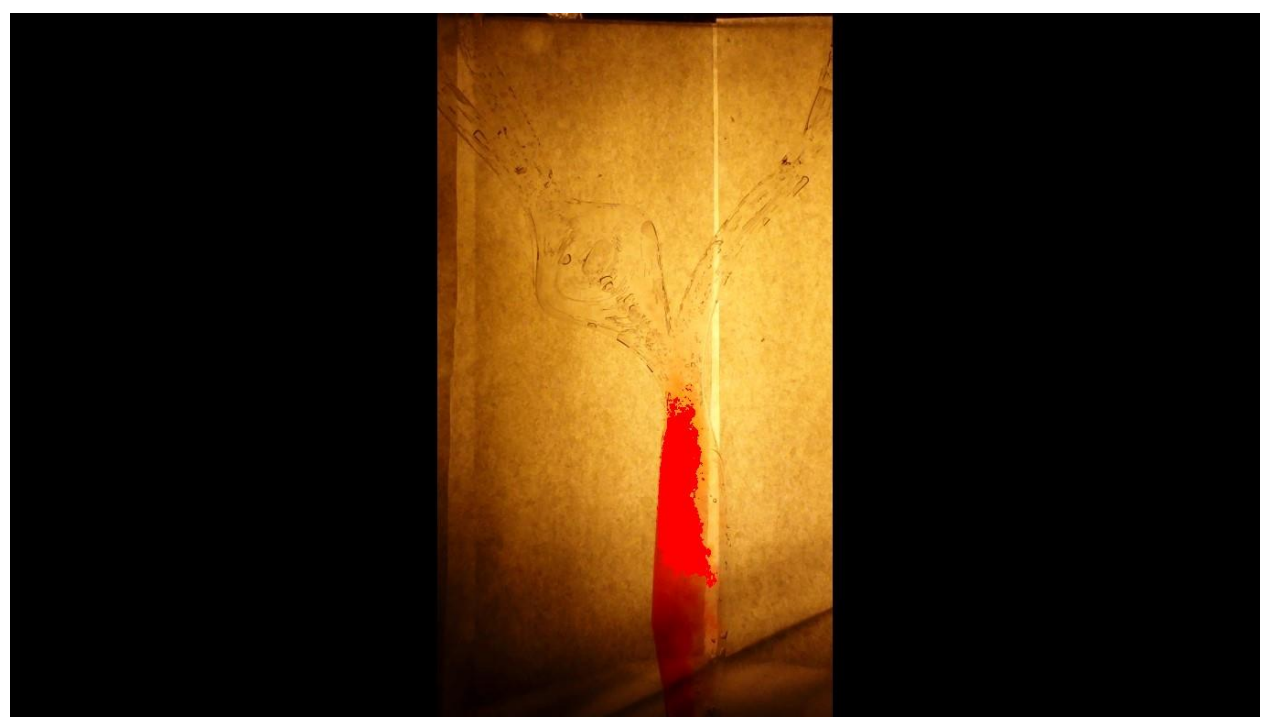

Figure 50: Run 3, Frame 3.

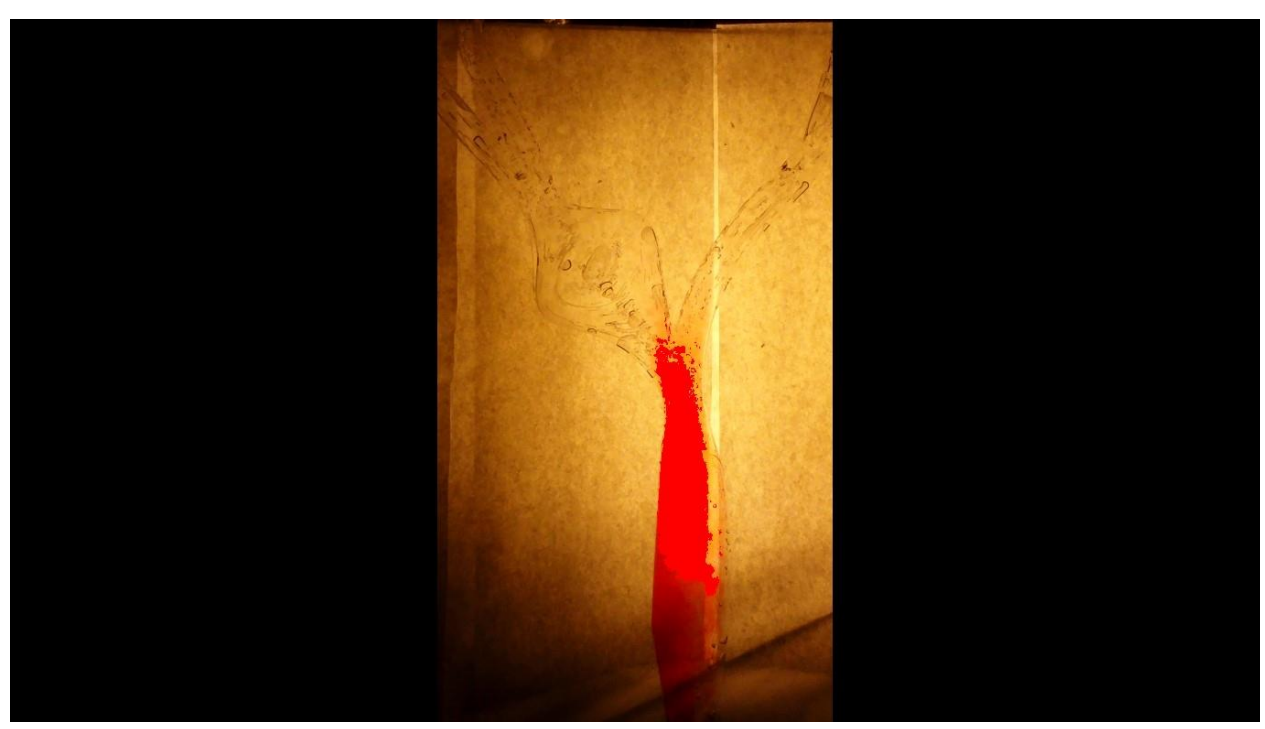

Figure 51: Run 3, Frame 4. 


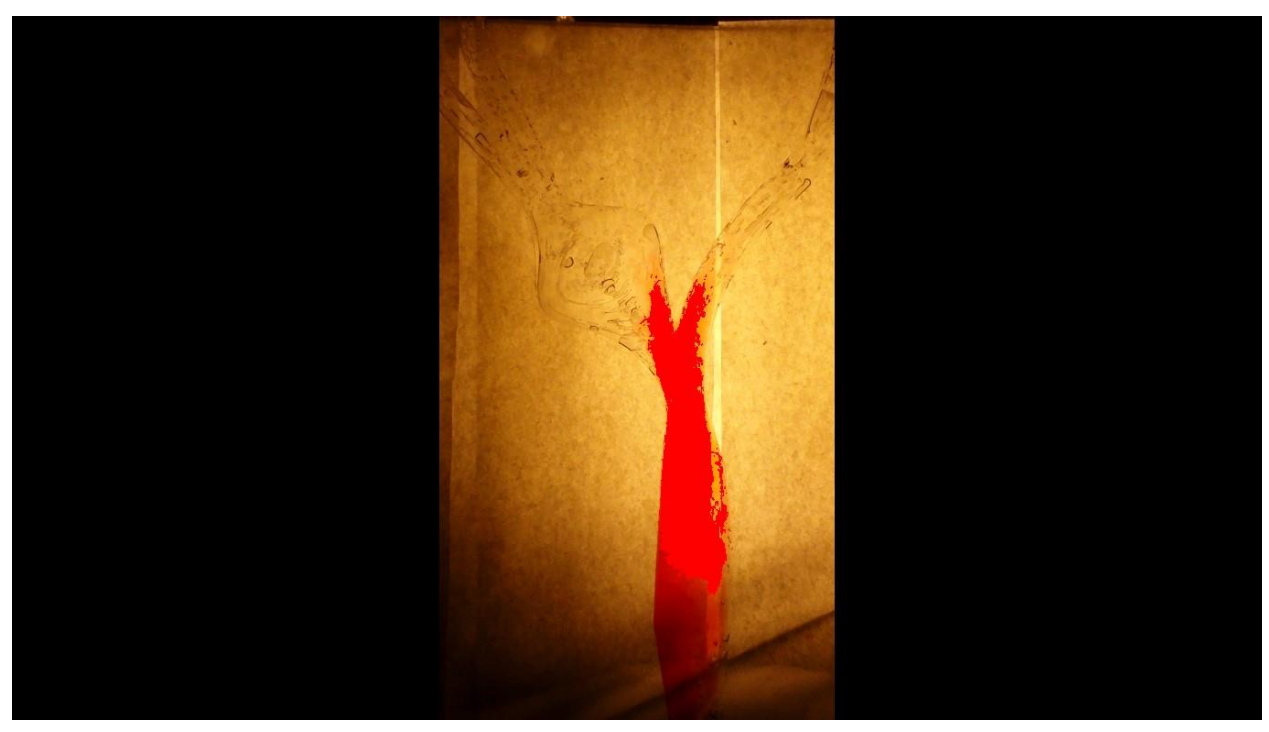

Figure 52: Run 3, Frame 5.

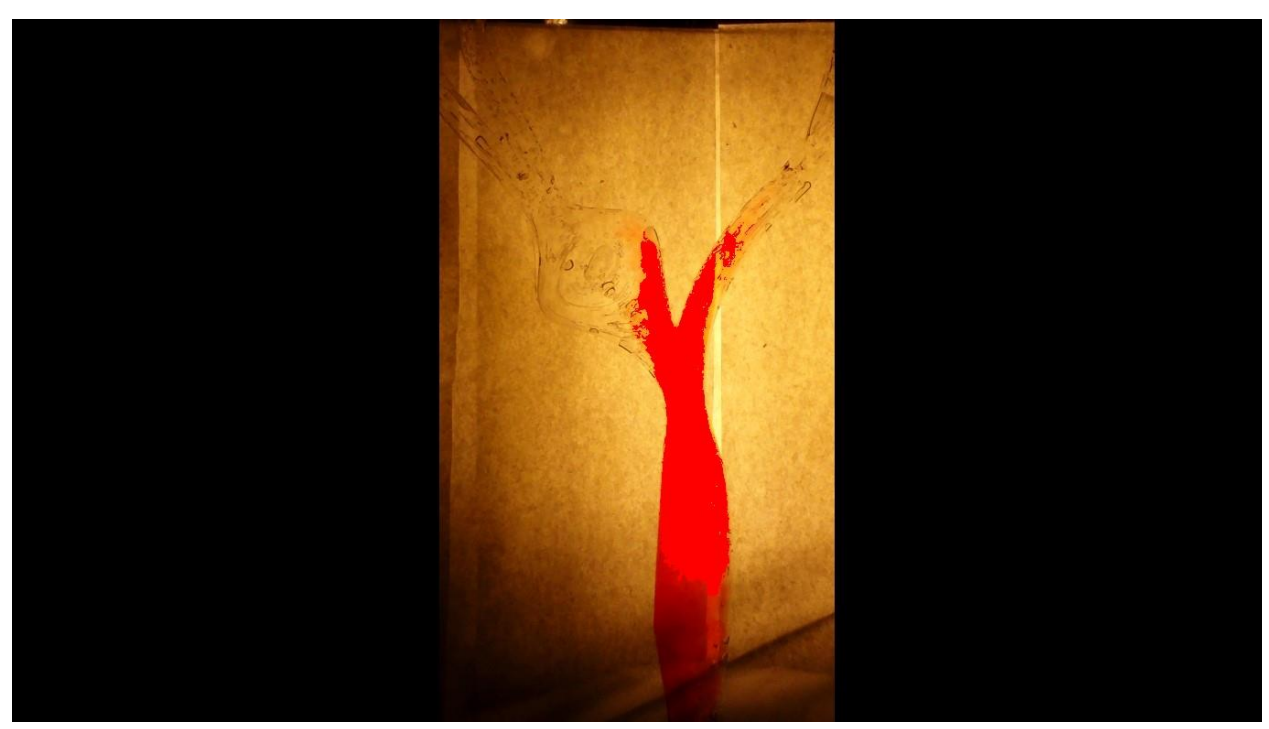

Figure 53: Run 3, Frame 6. 


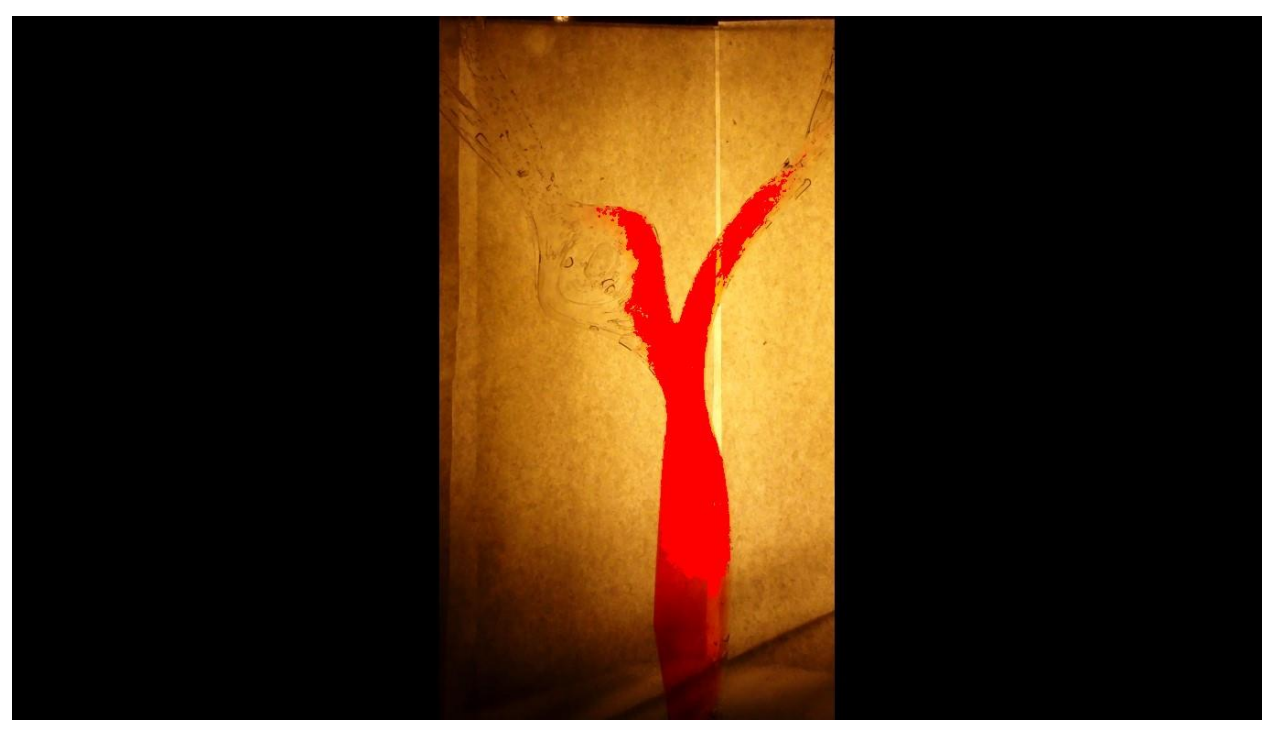

Figure 54:Run 3, Frame 7.

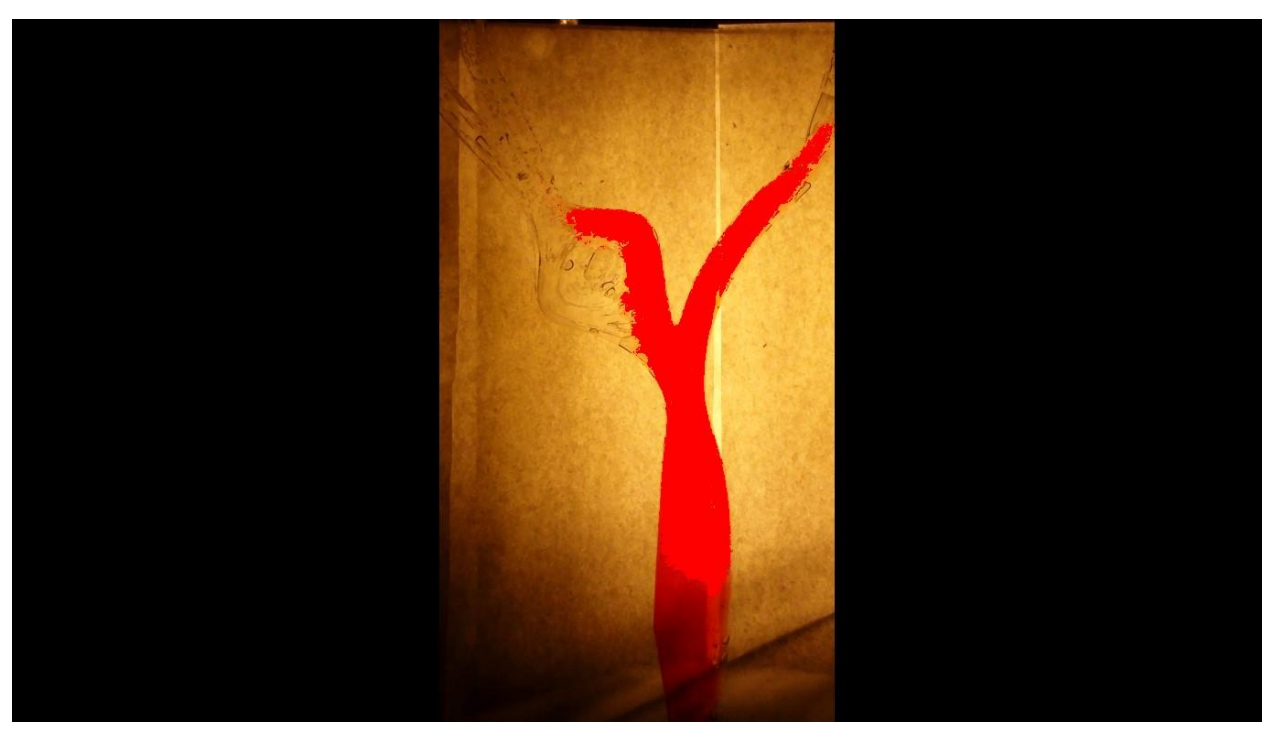

Figure 55: Run 3, Frame 8. 


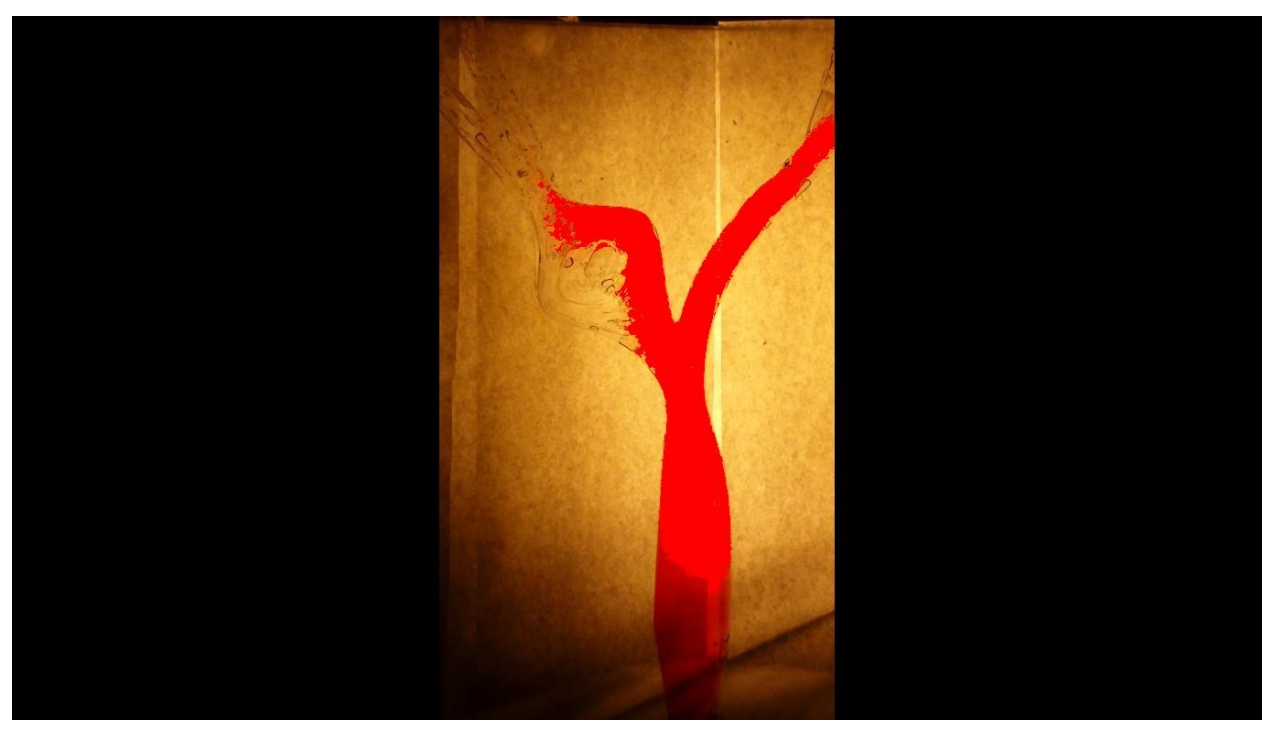

Figure 56: Run 3, Frame 9.

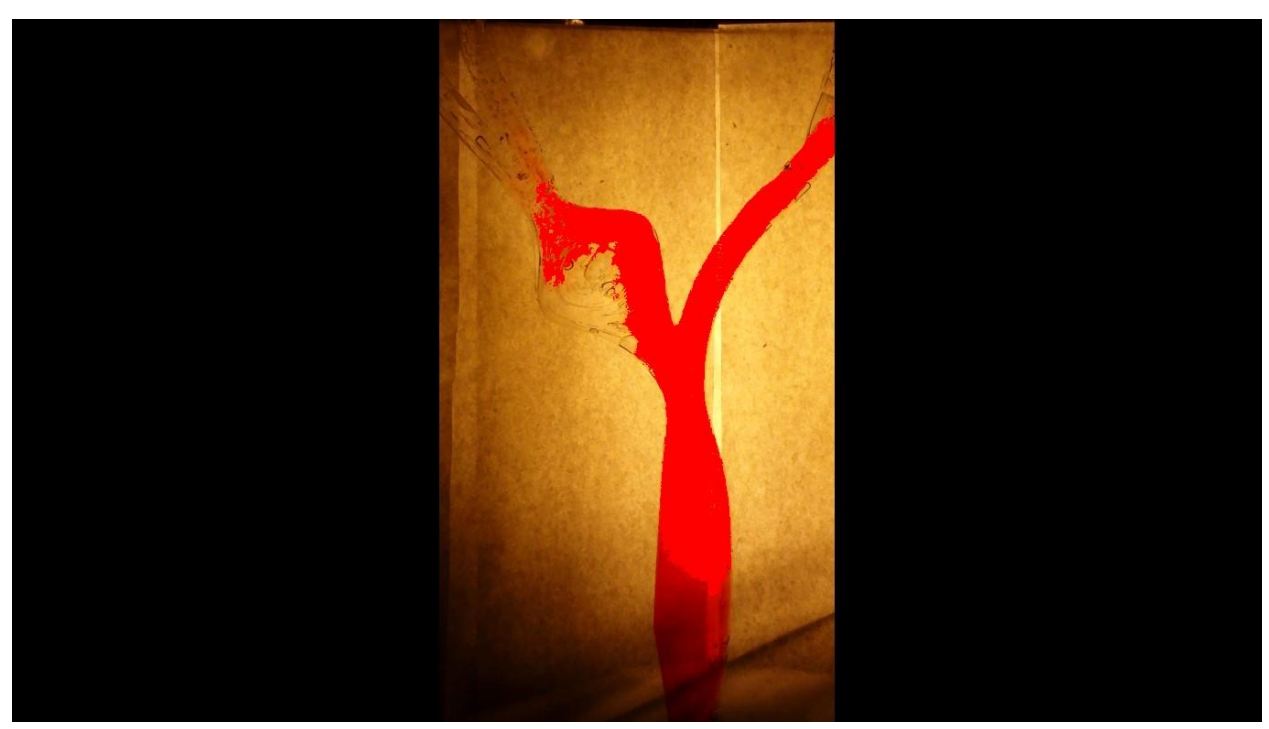

Figure 57: Run 3, Frame 10. 


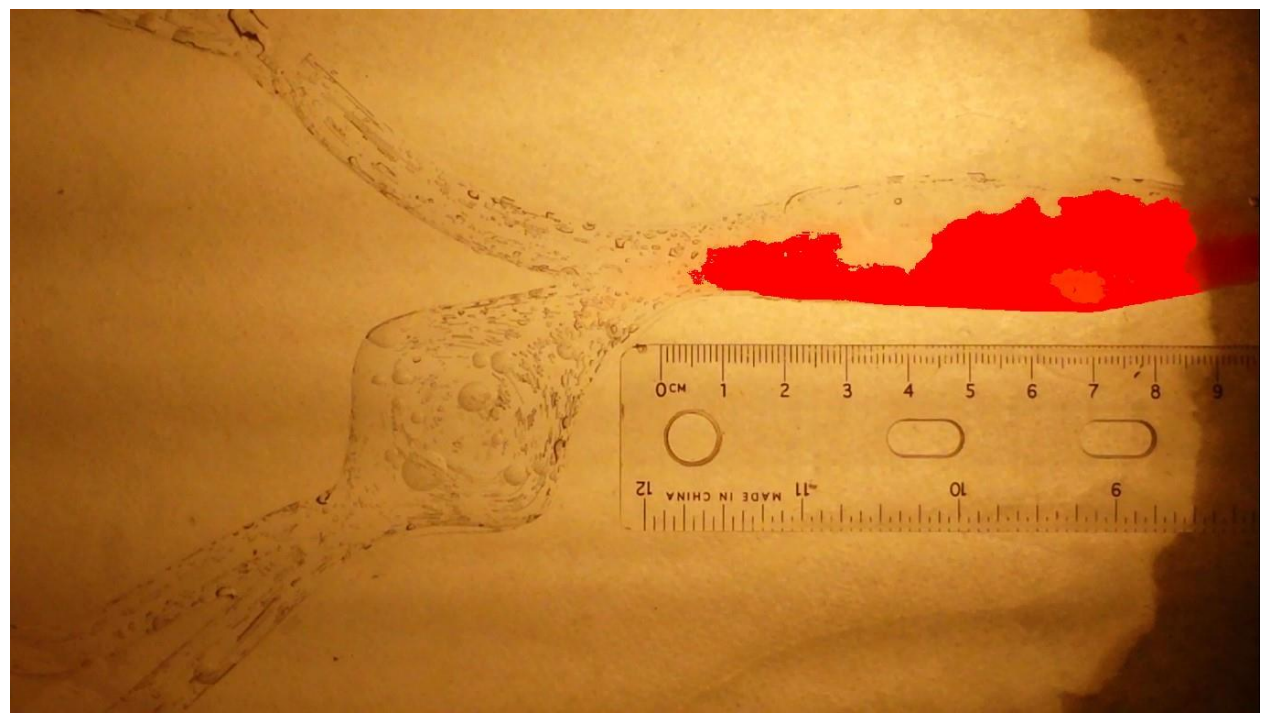

Figure 58: Run 4, Frame 1.

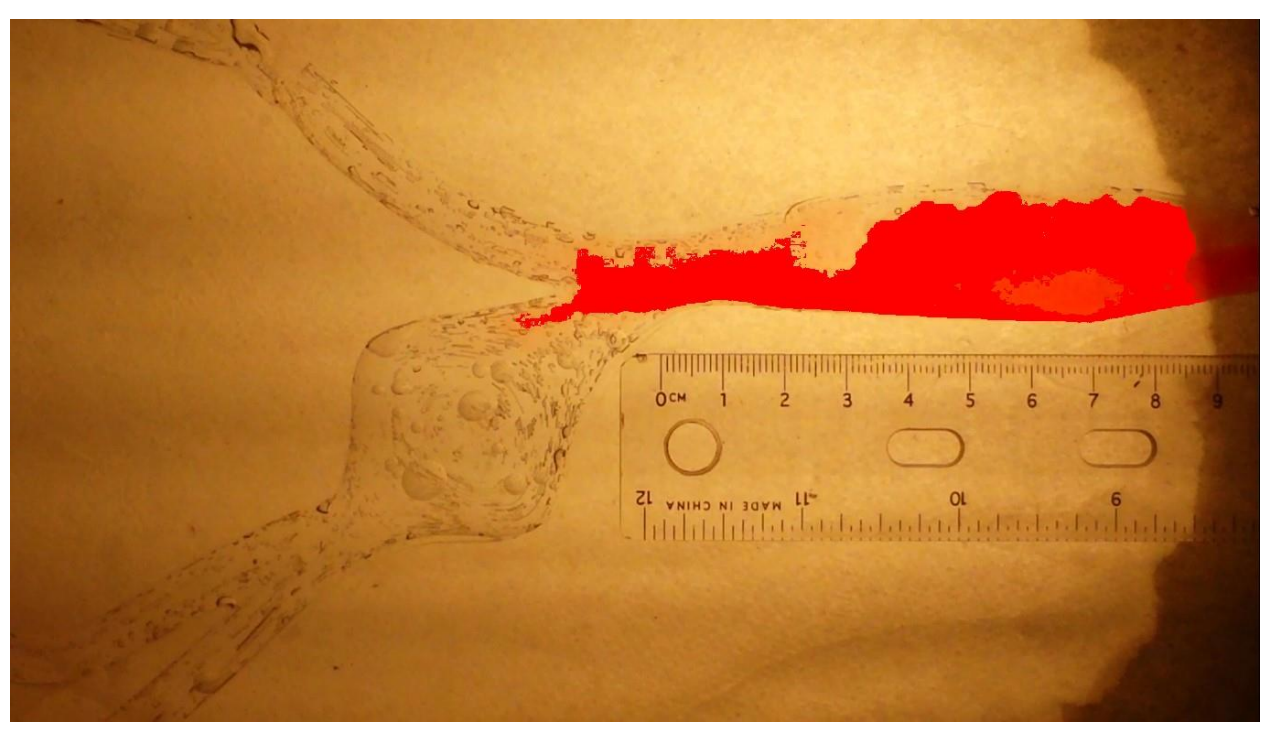

Figure 59: Run 4, Frame 2. 


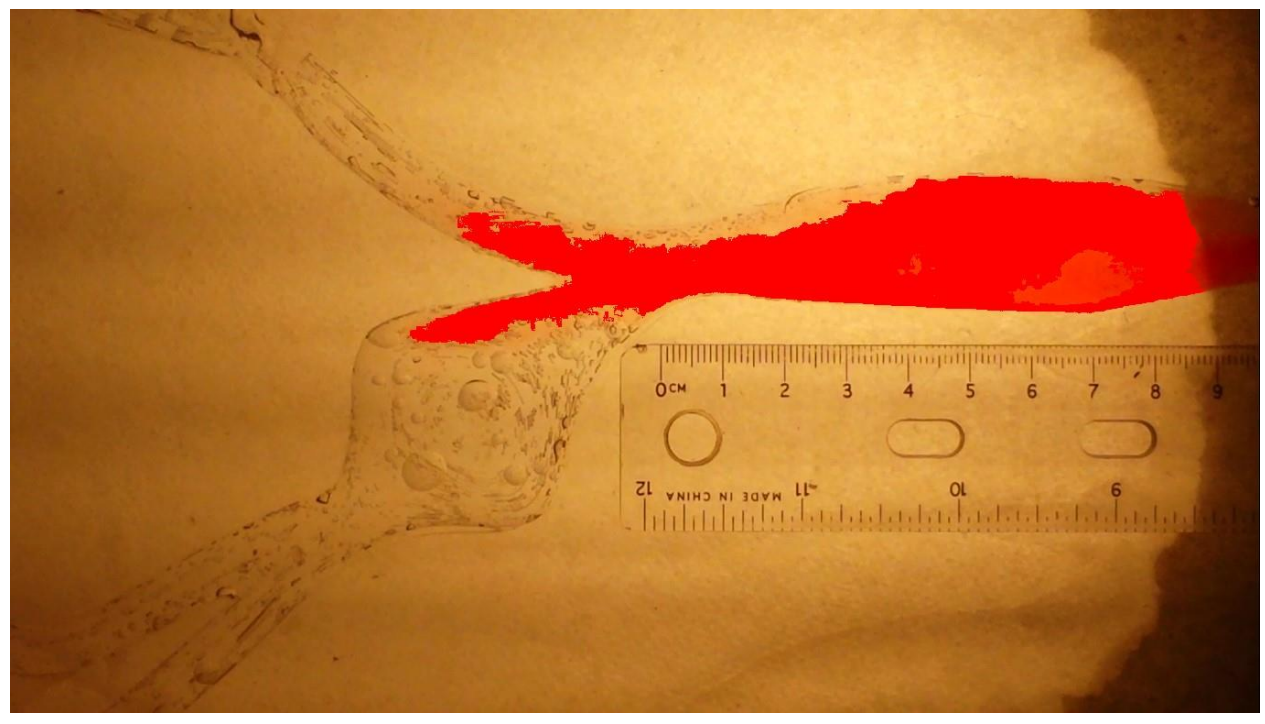

Figure 60: Run 4, Frame 3.

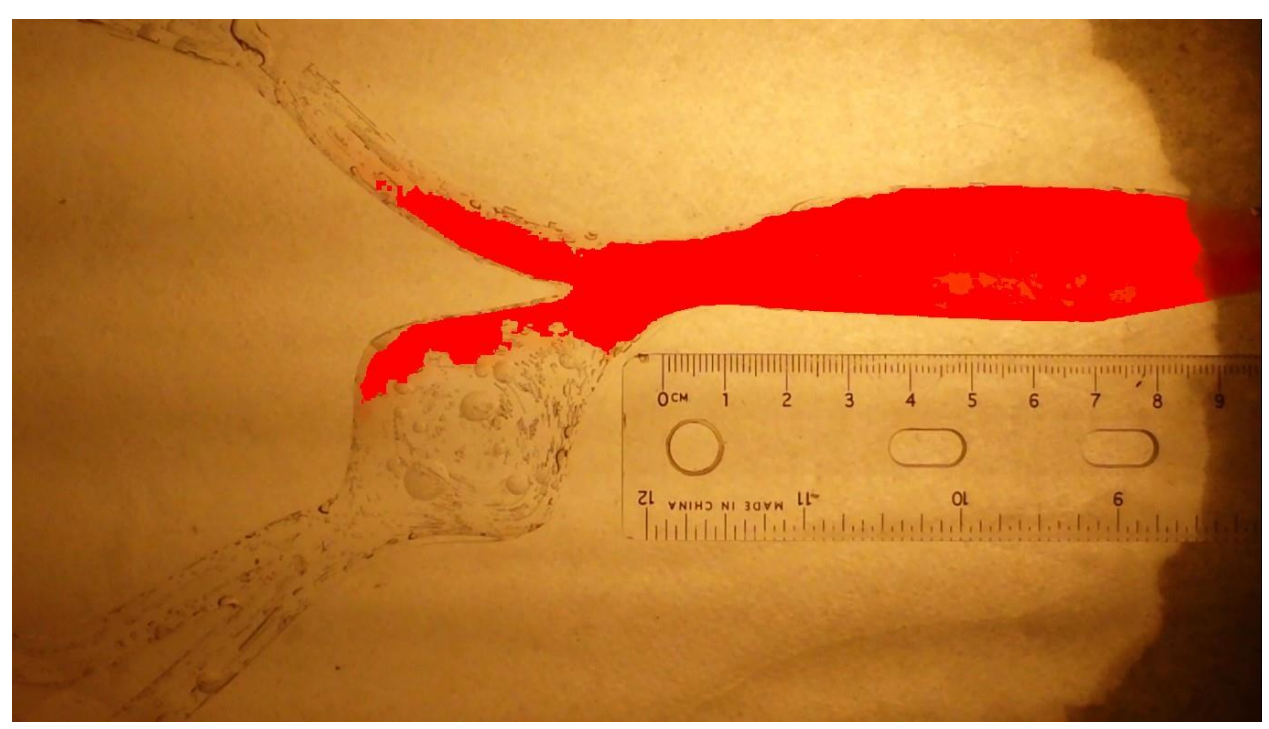

Figure 61: Run 4, Frame 4. 


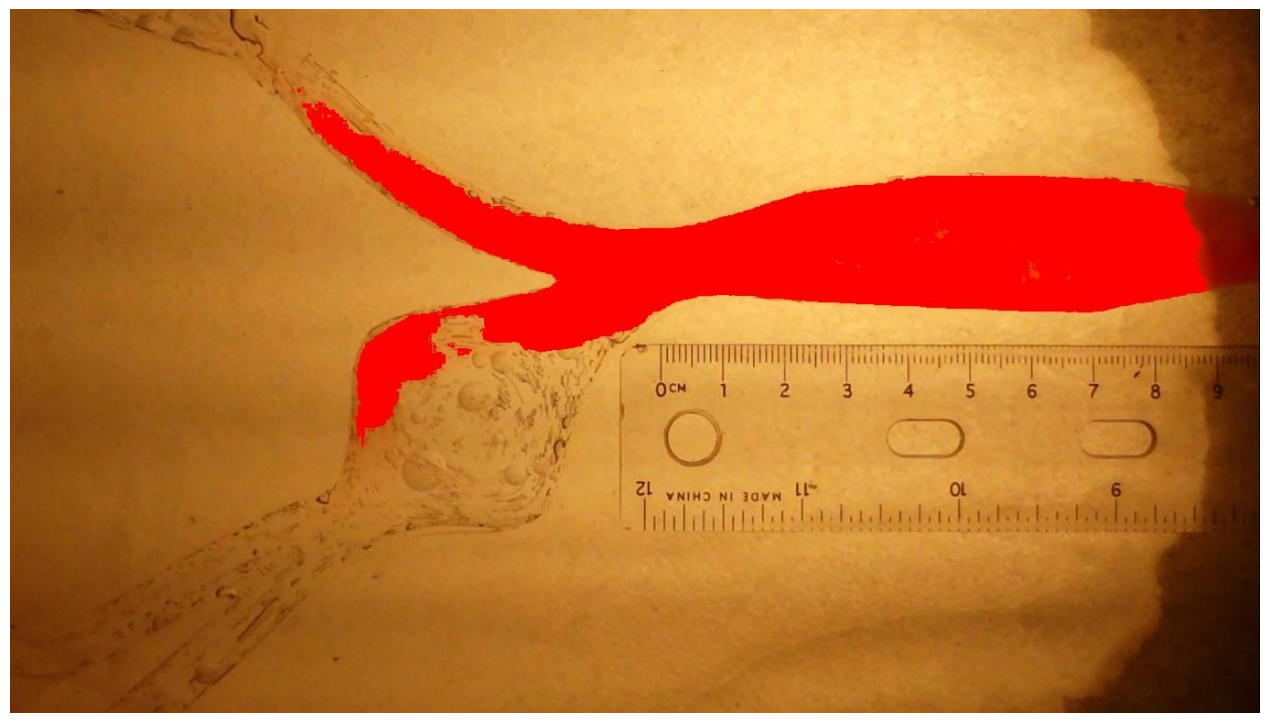

Figure 62: Run 4, Frame 5.

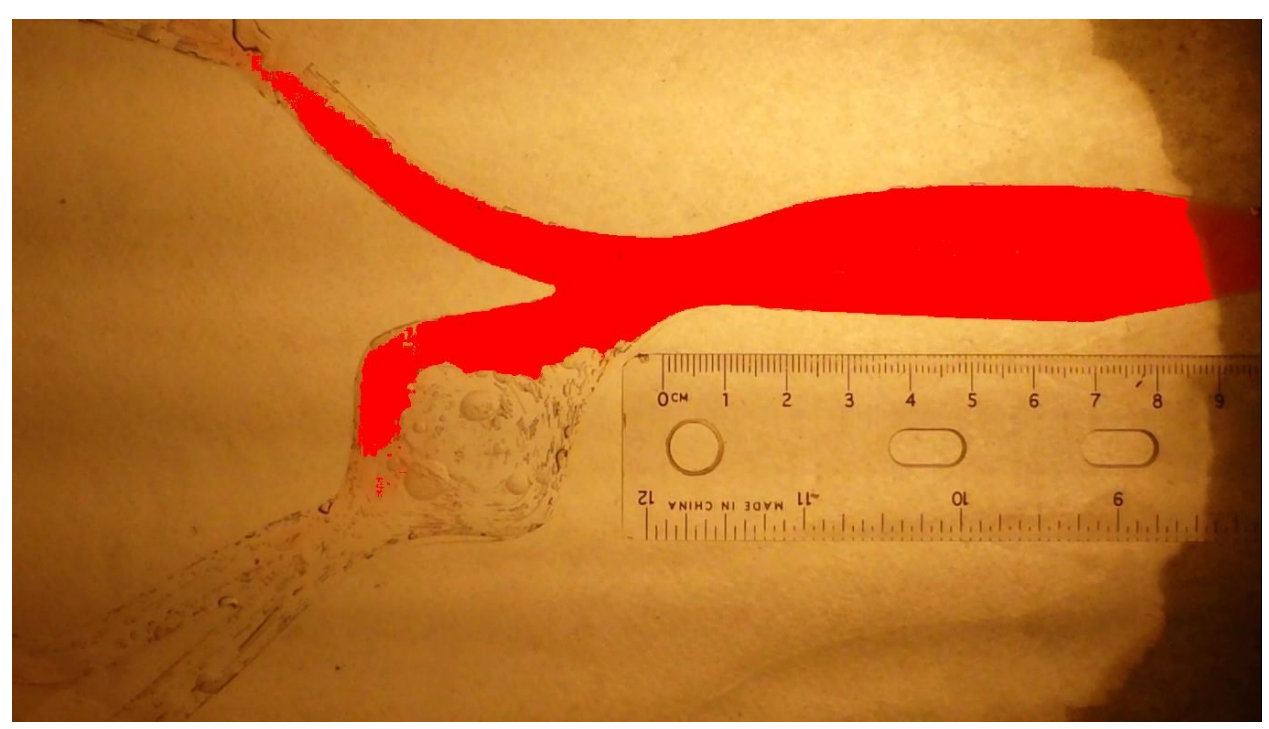

Figure 63: Run 4, Frame 6. 


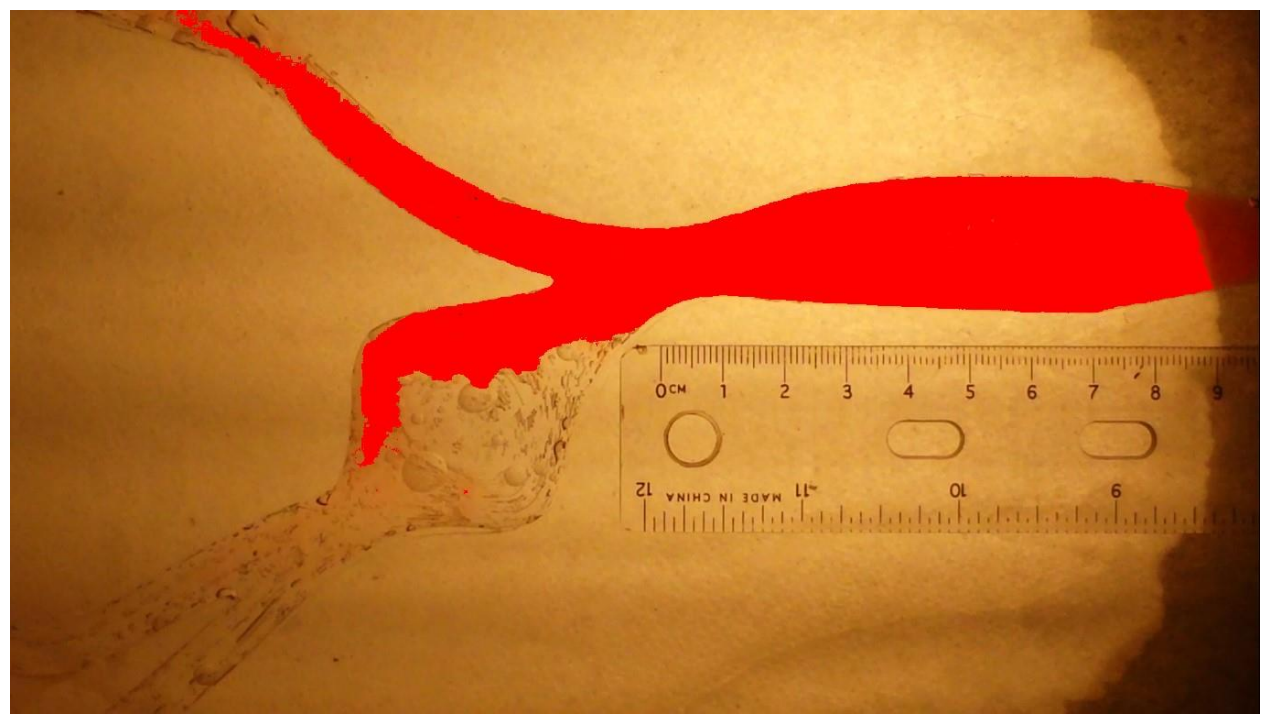

Figure 64: Run 4, Frame 7.

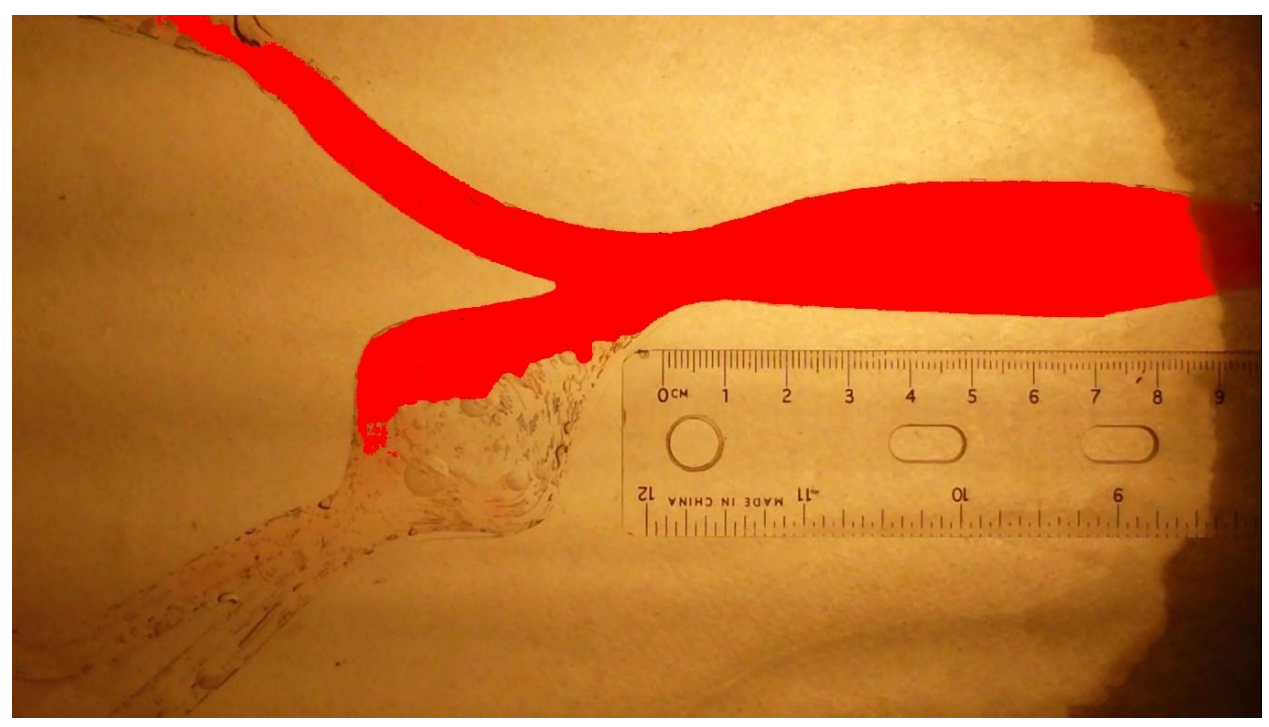

Figure 65: Run 4, Frame 8. 


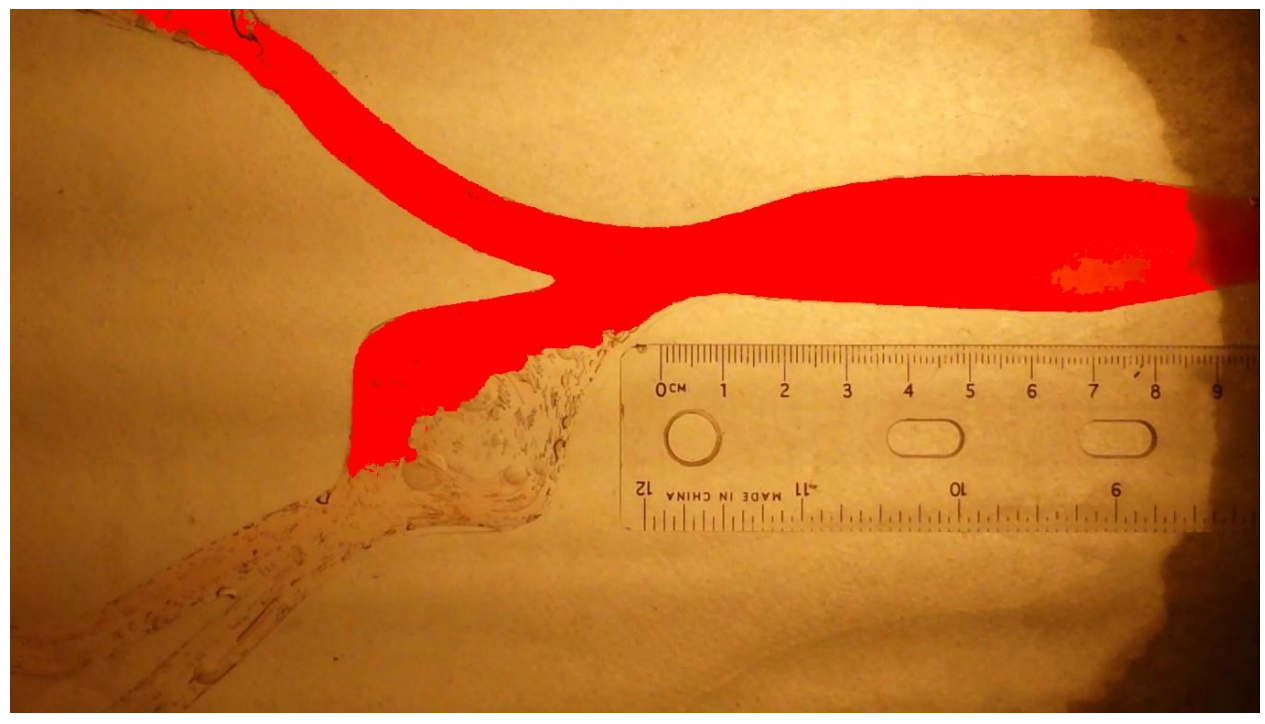

Figure 66: Run 4, Frame 9.

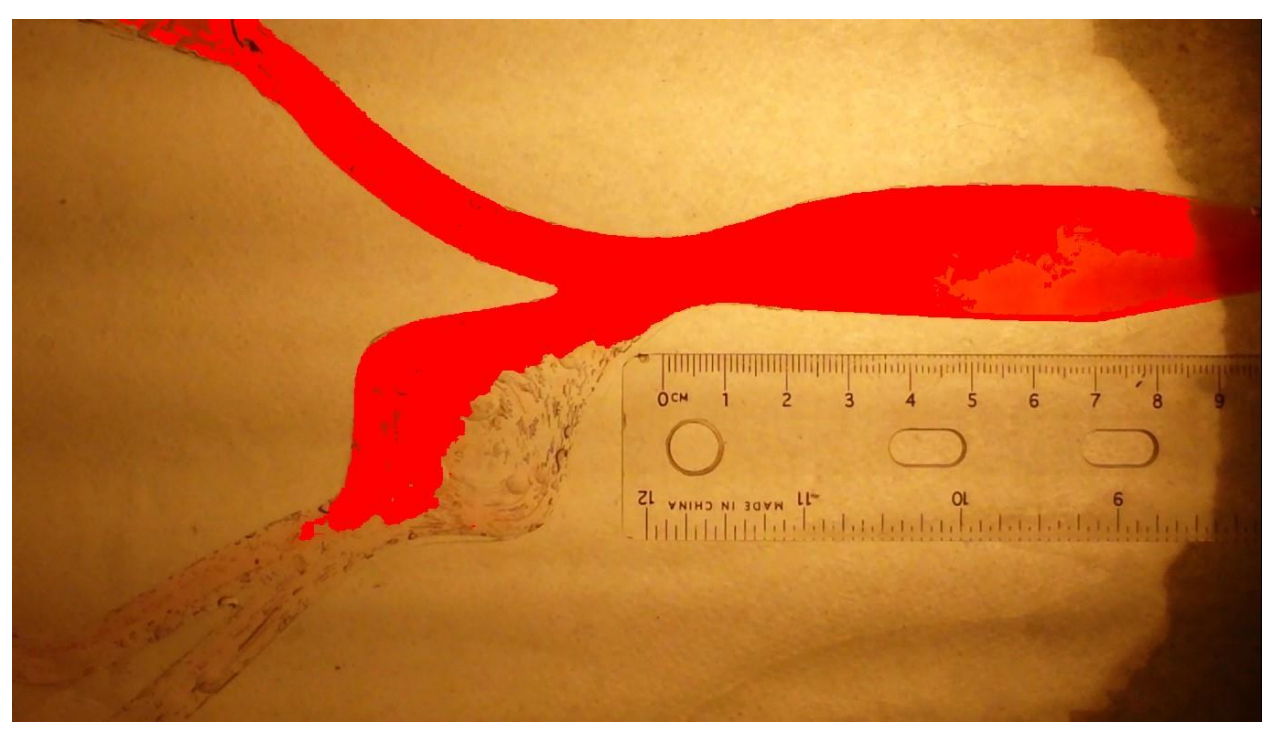

Figure 67: Run 4, Frame 10. 


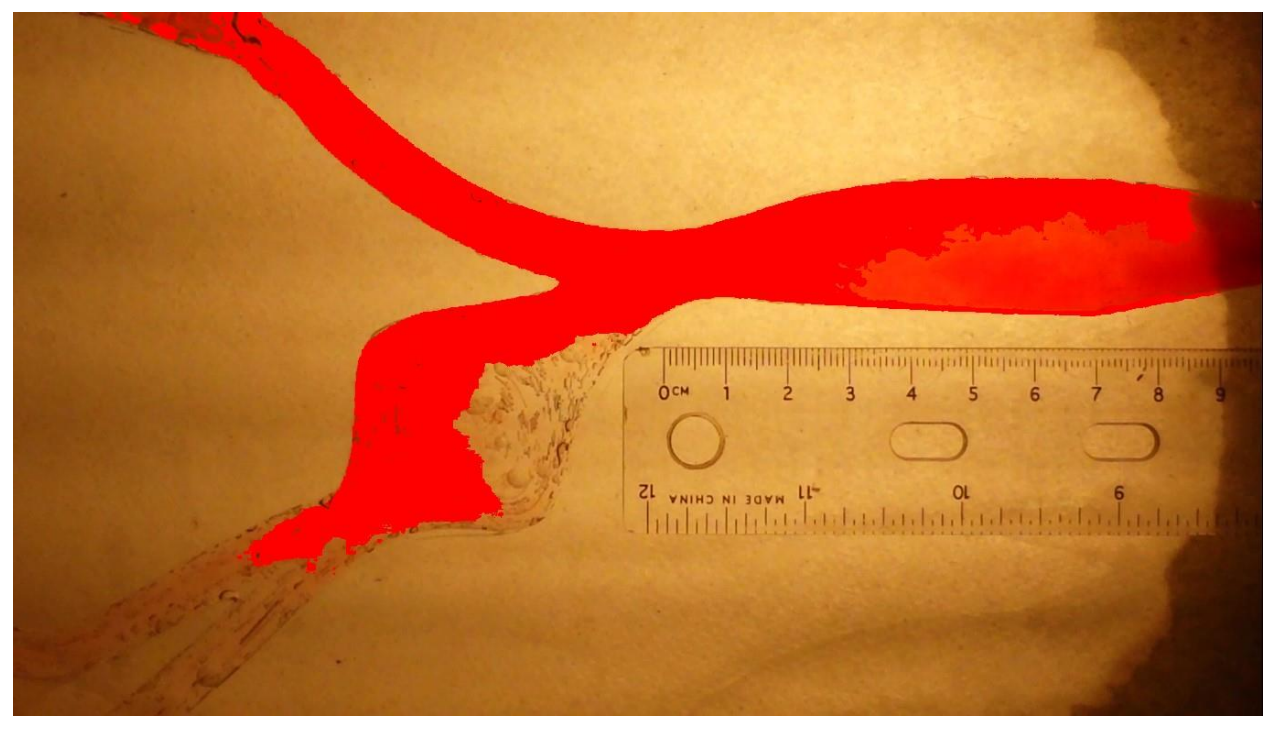

Figure 68: Run 4, Frame 11.

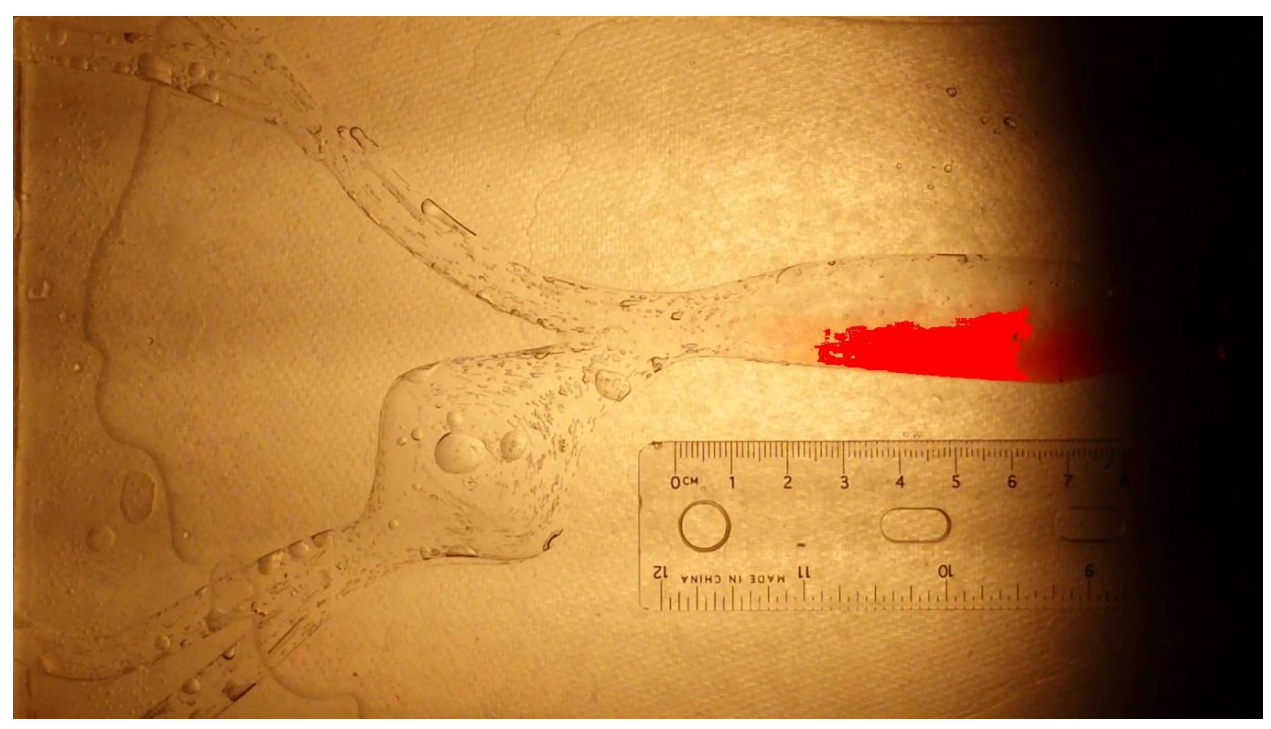

Figure 69: Run 5, Frame 1. 


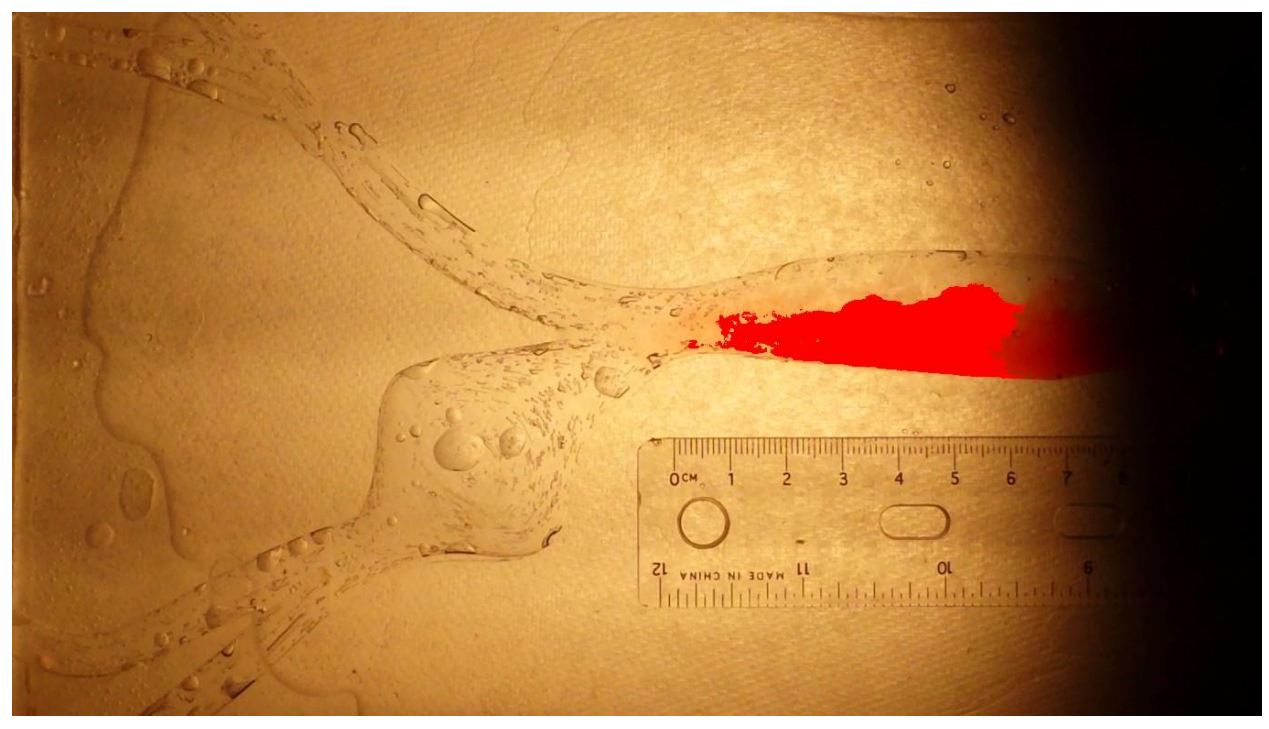

Figure 70: Run 5, Frame 2.

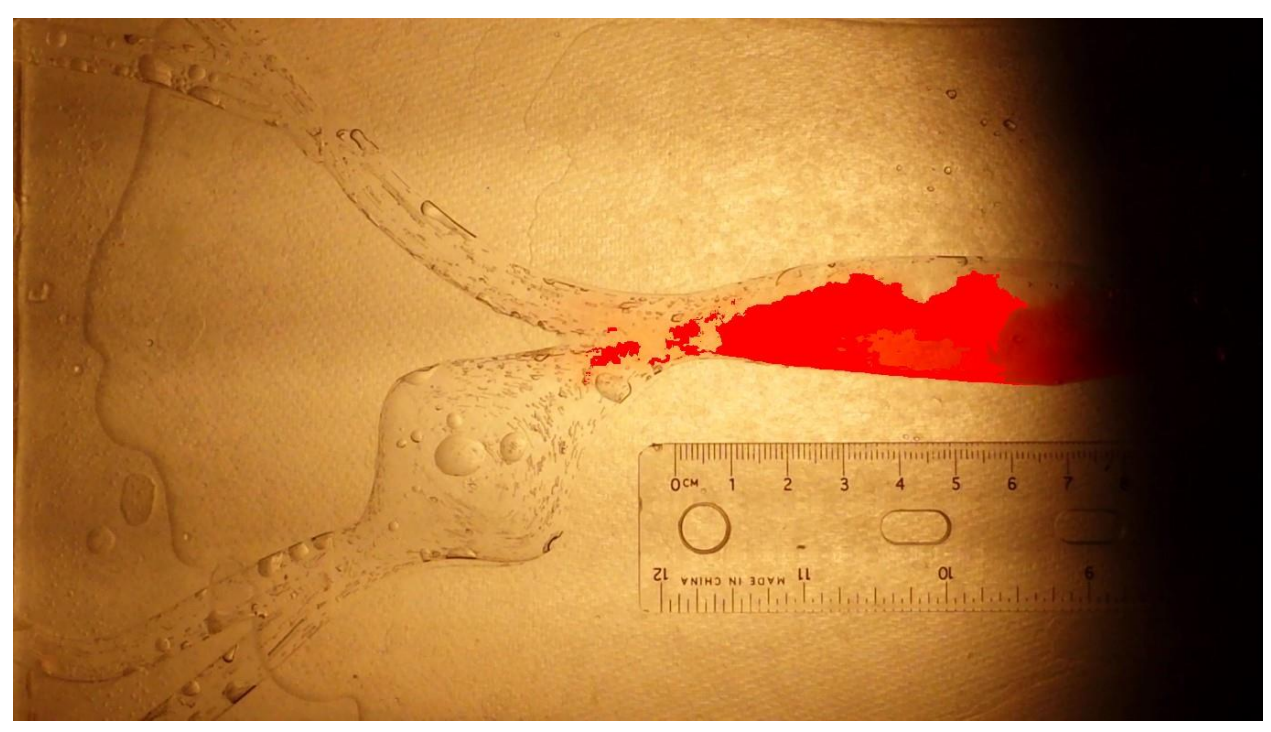

Figure 71: Run 5, Frame 3. 


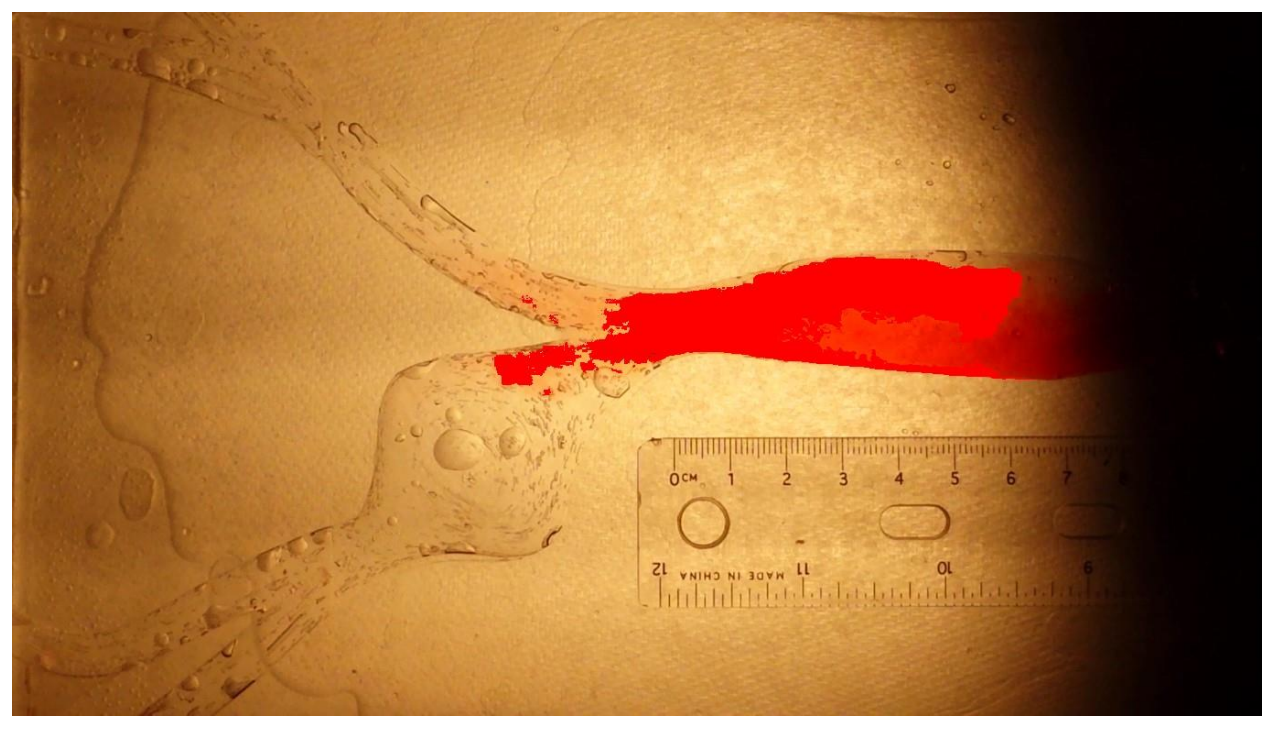

Figure 72: Run 5, Frame 4.

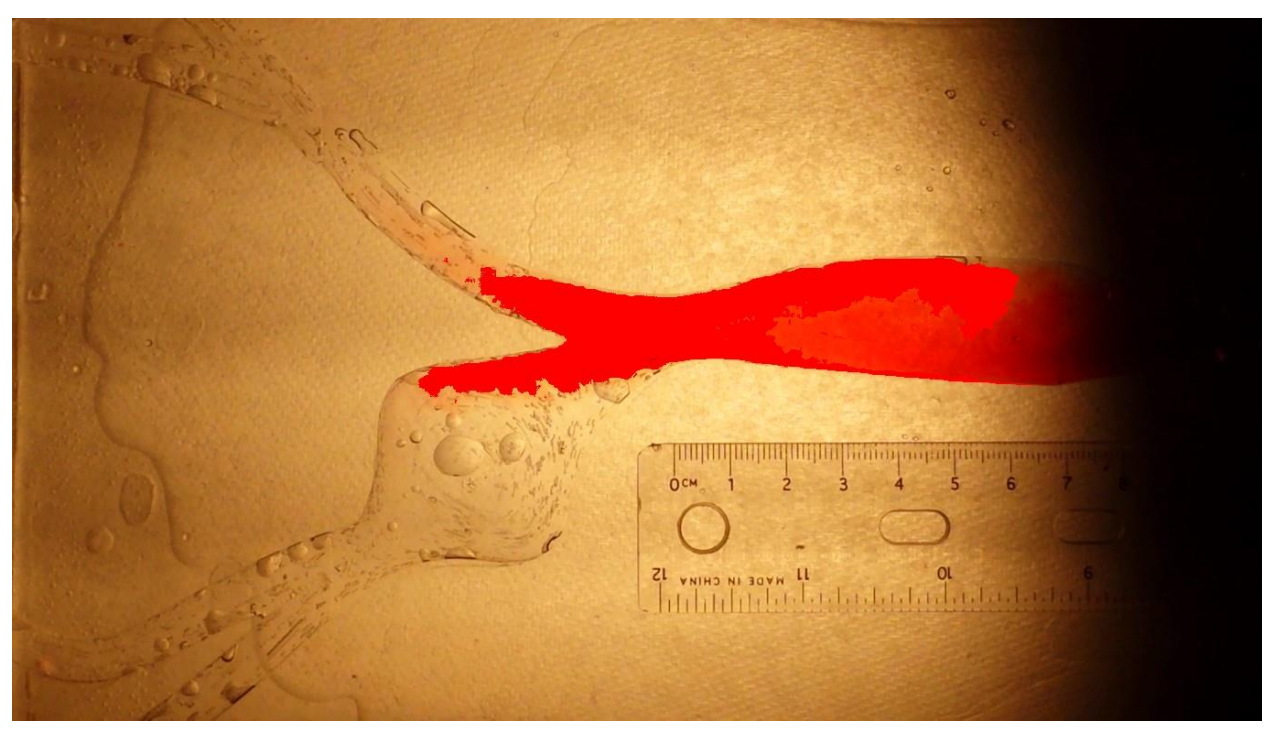

Figure 73: Run 5, Frame 5. 


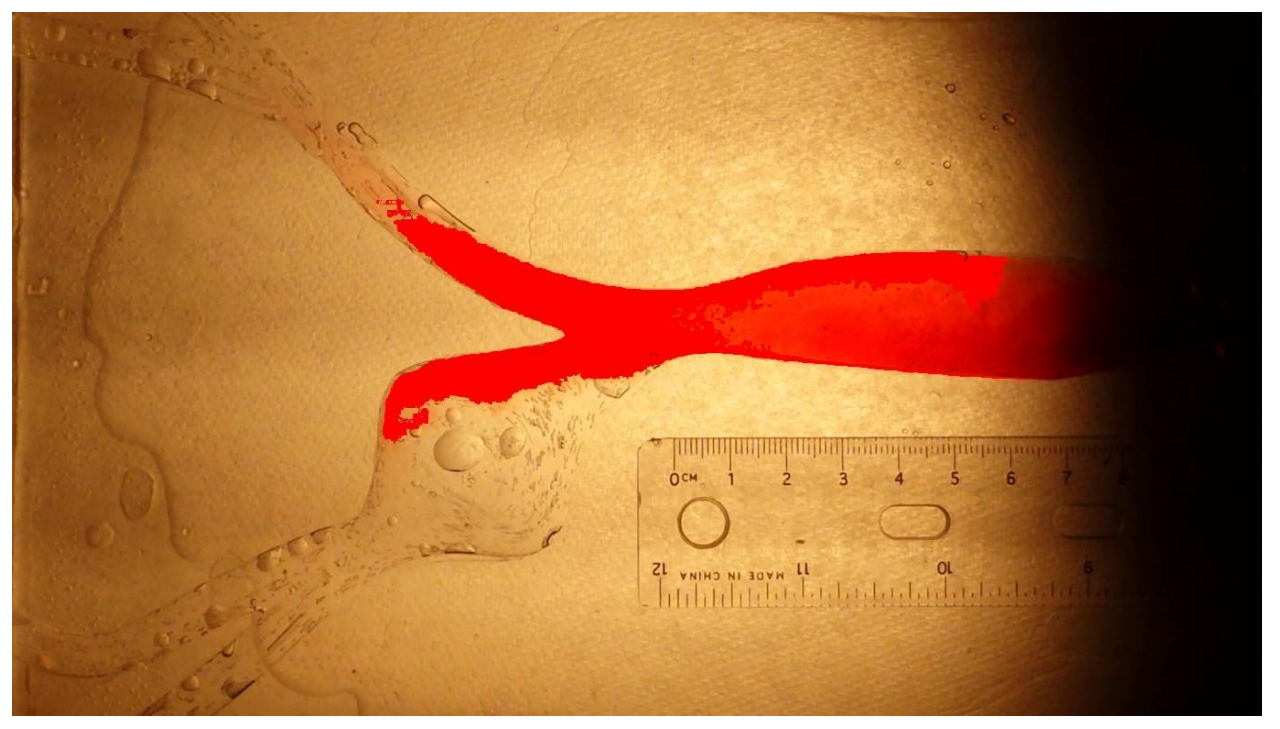

Figure 74: Run 5, Frame 6.

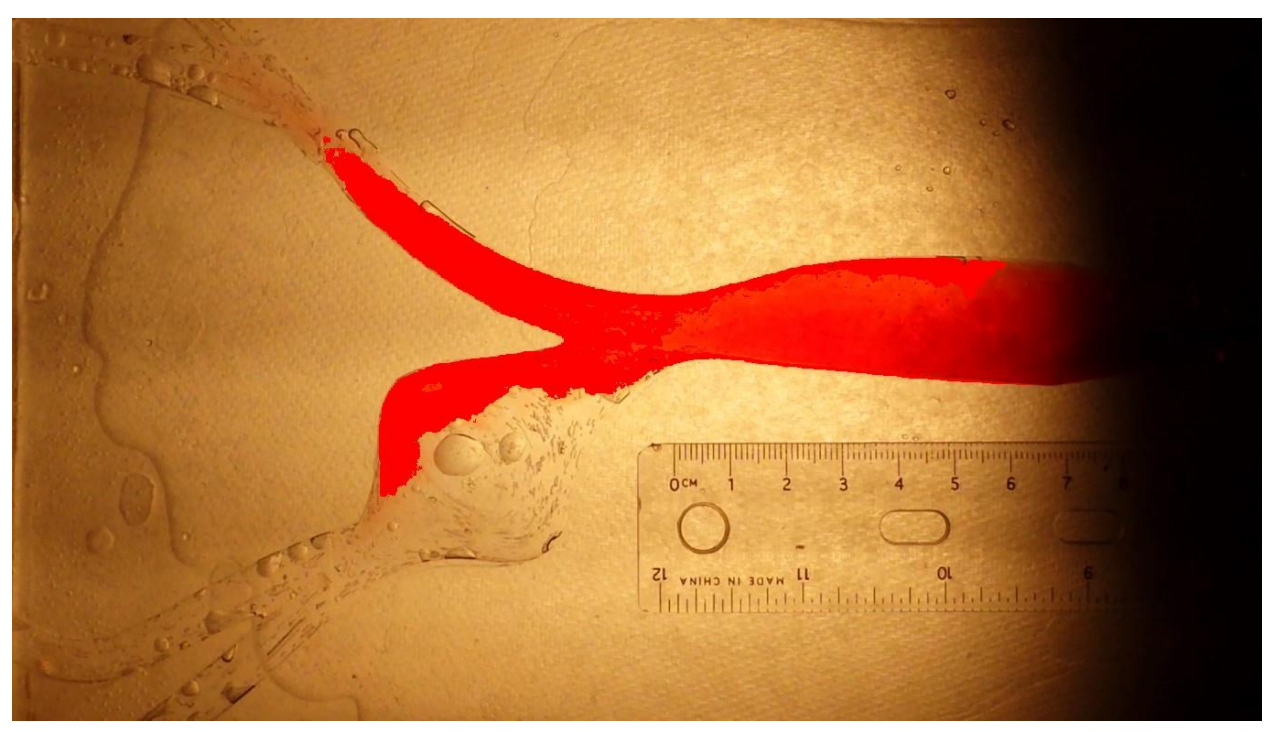

Figure 75: Run 5, Frame 7. 


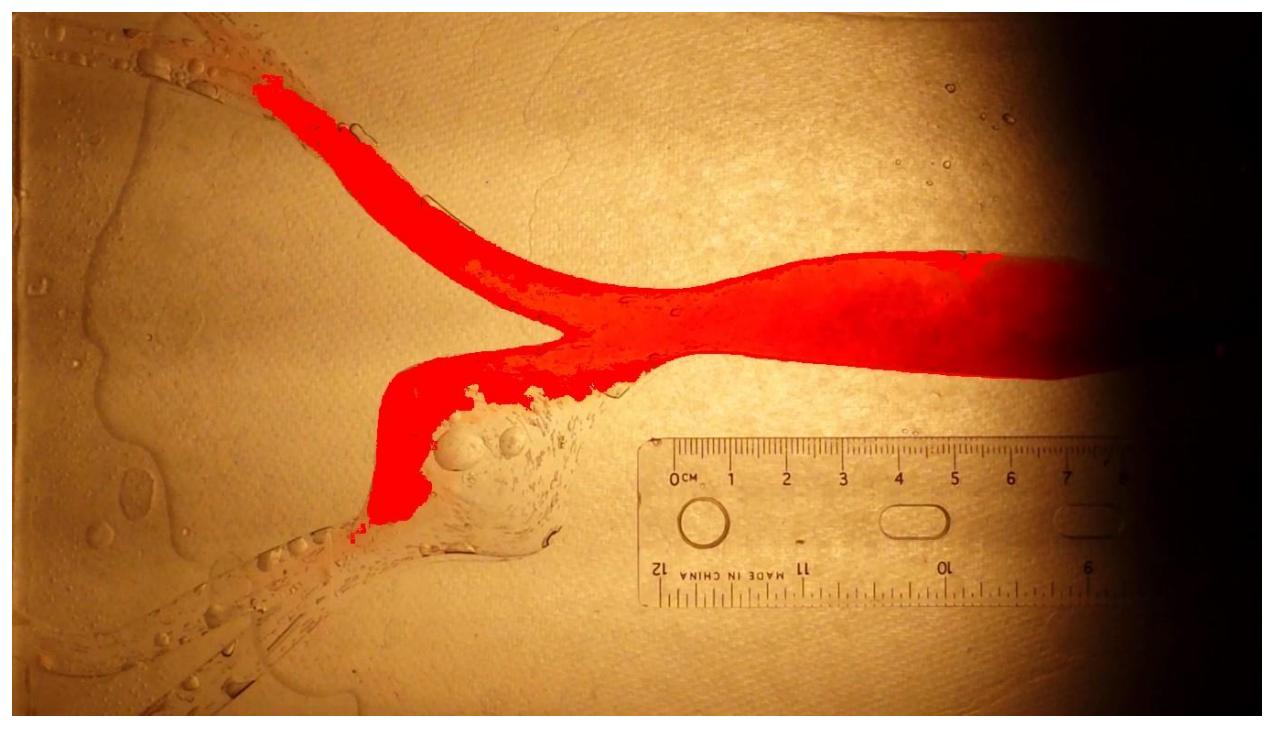

Figure 76: Run 5, Frame 8.

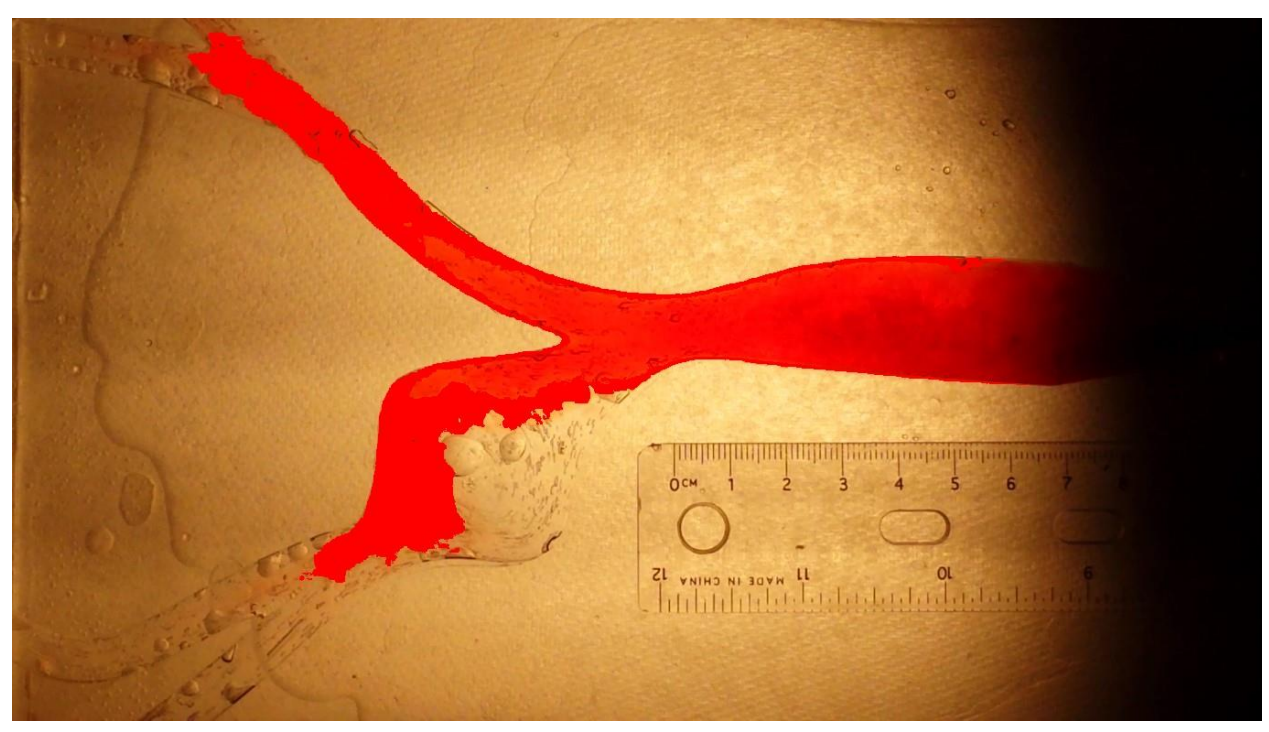

Figure 77: Run 5, Frame 9. 


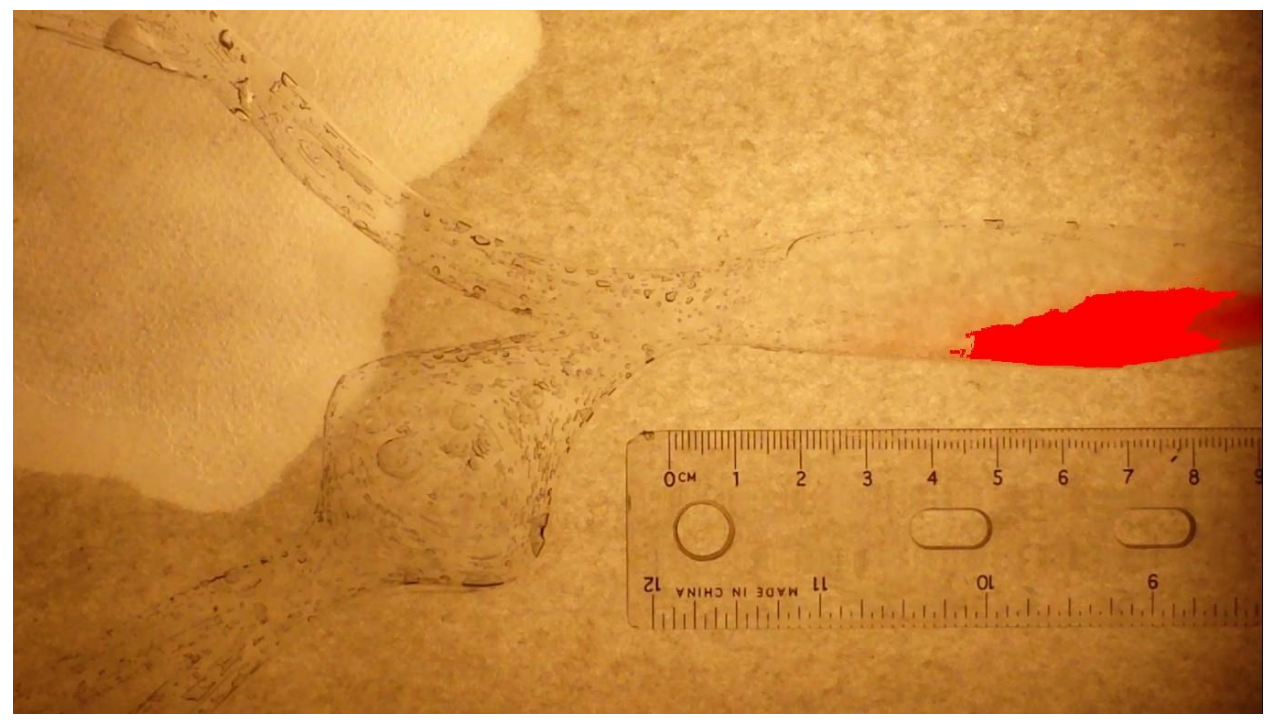

Figure 78: Run 6, Frame 1.

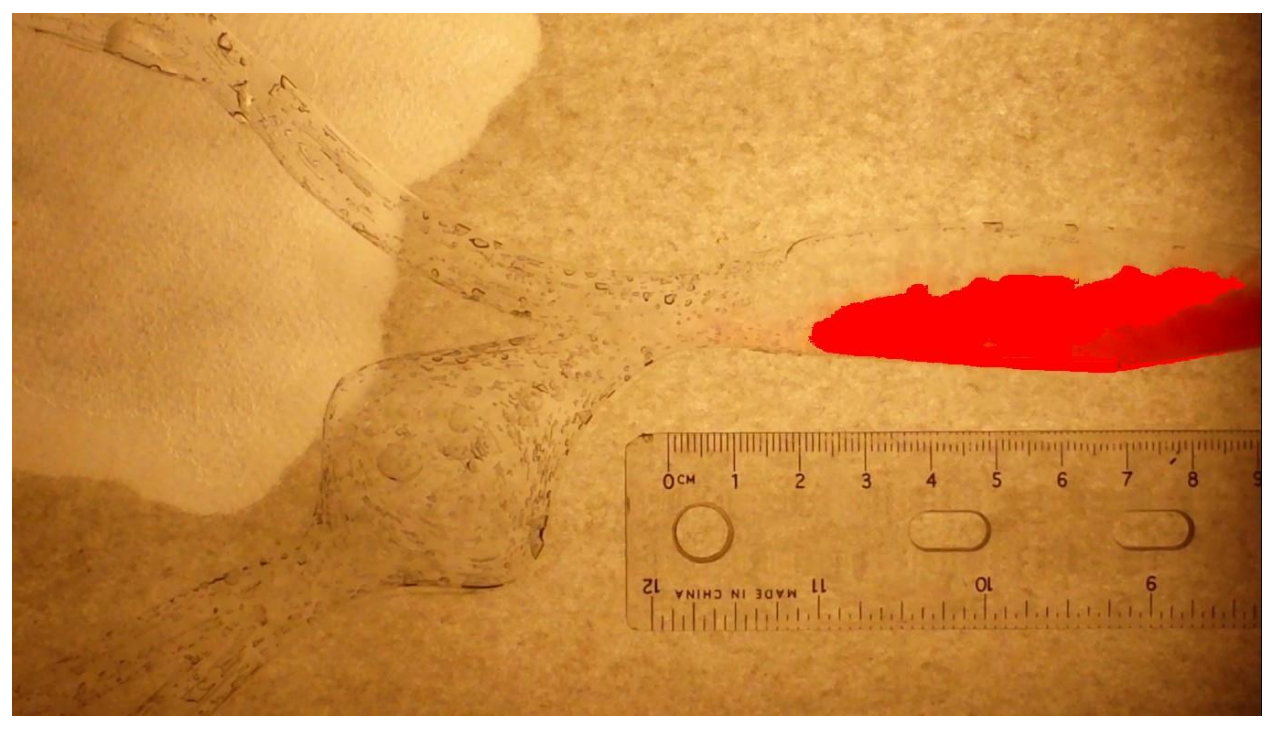

Figure 79: Run 6, Frame 2. 


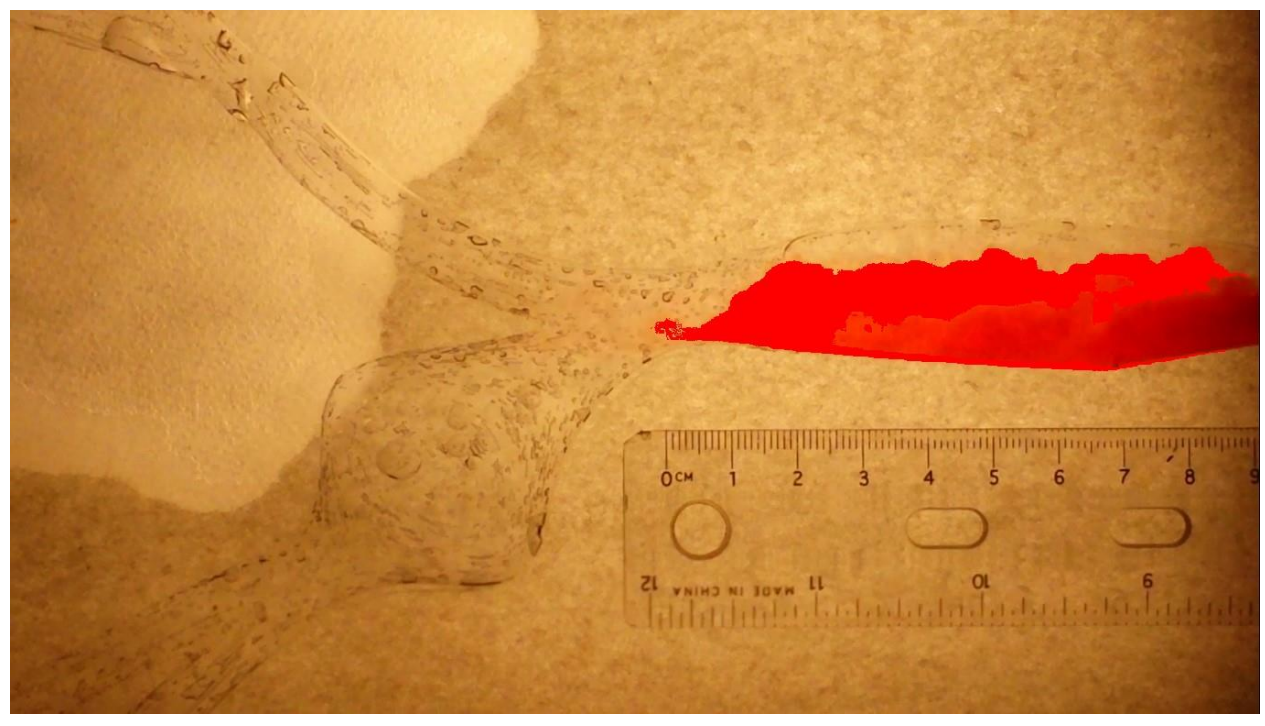

Figure 80: Run 6, Frame 3.

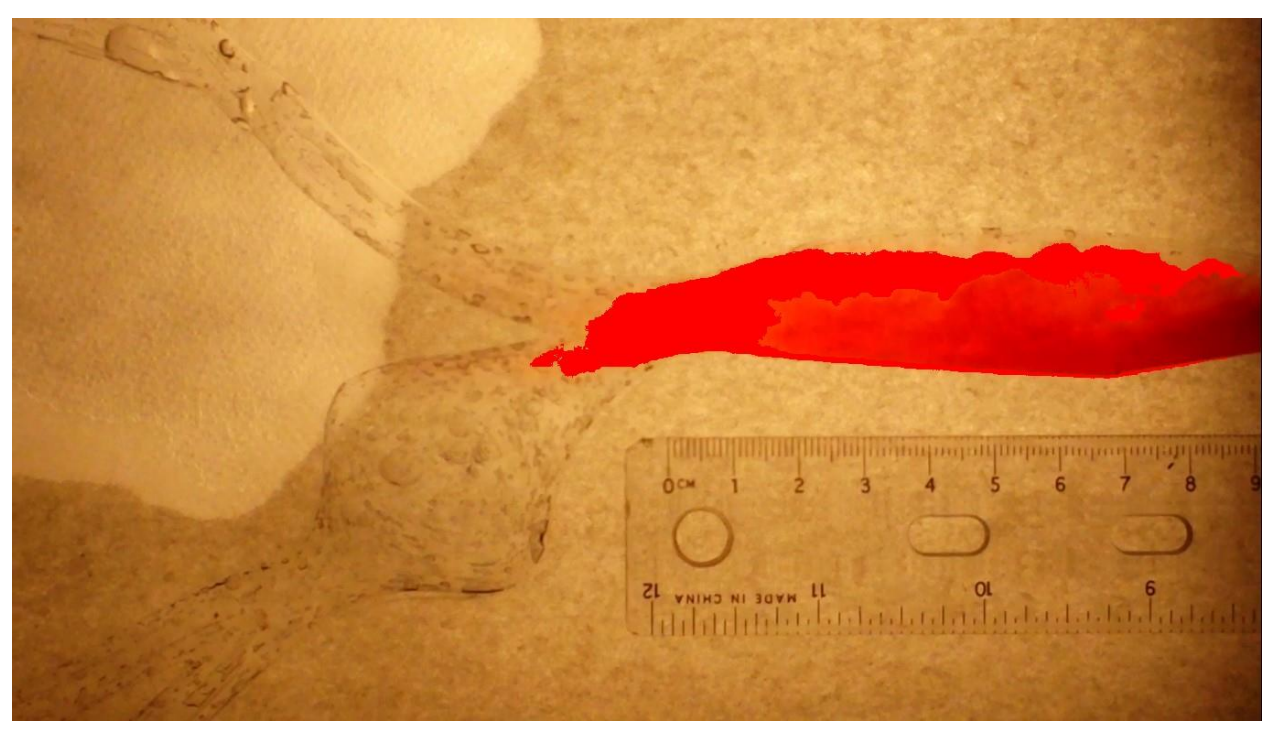

Figure 81: Run 6, Frame 4. 


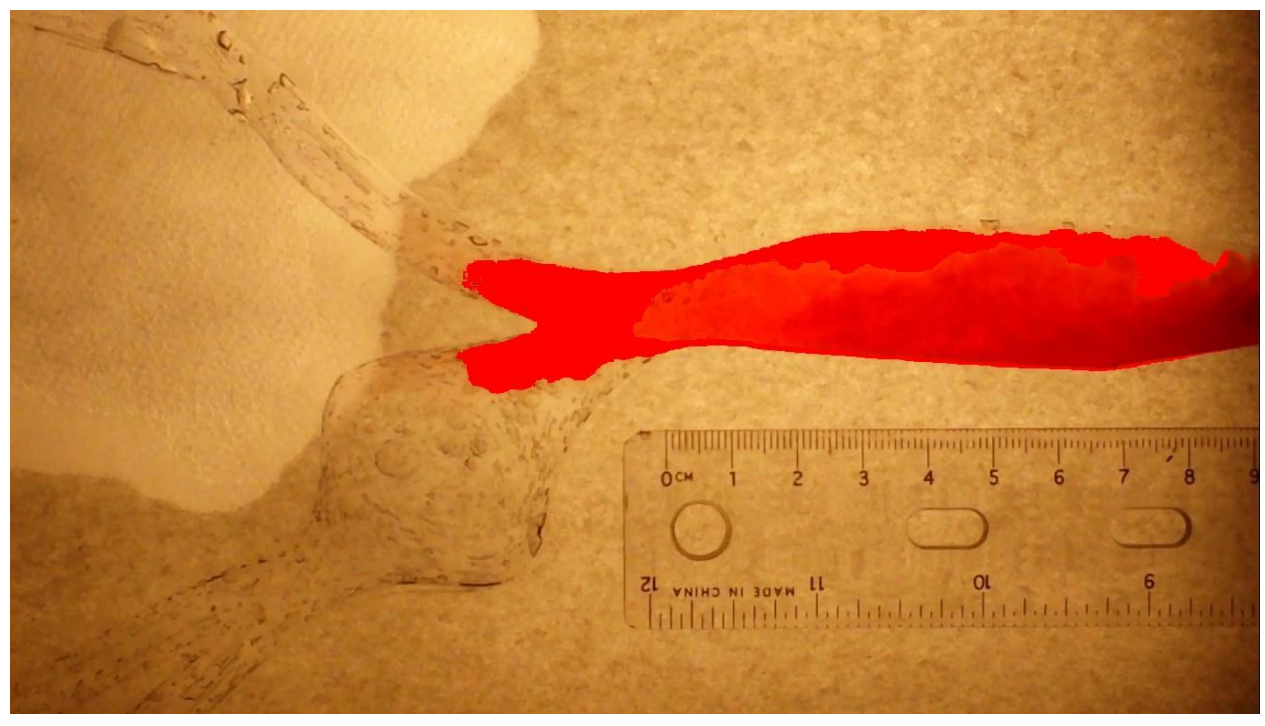

Figure 82: Run 6, Frame 5.

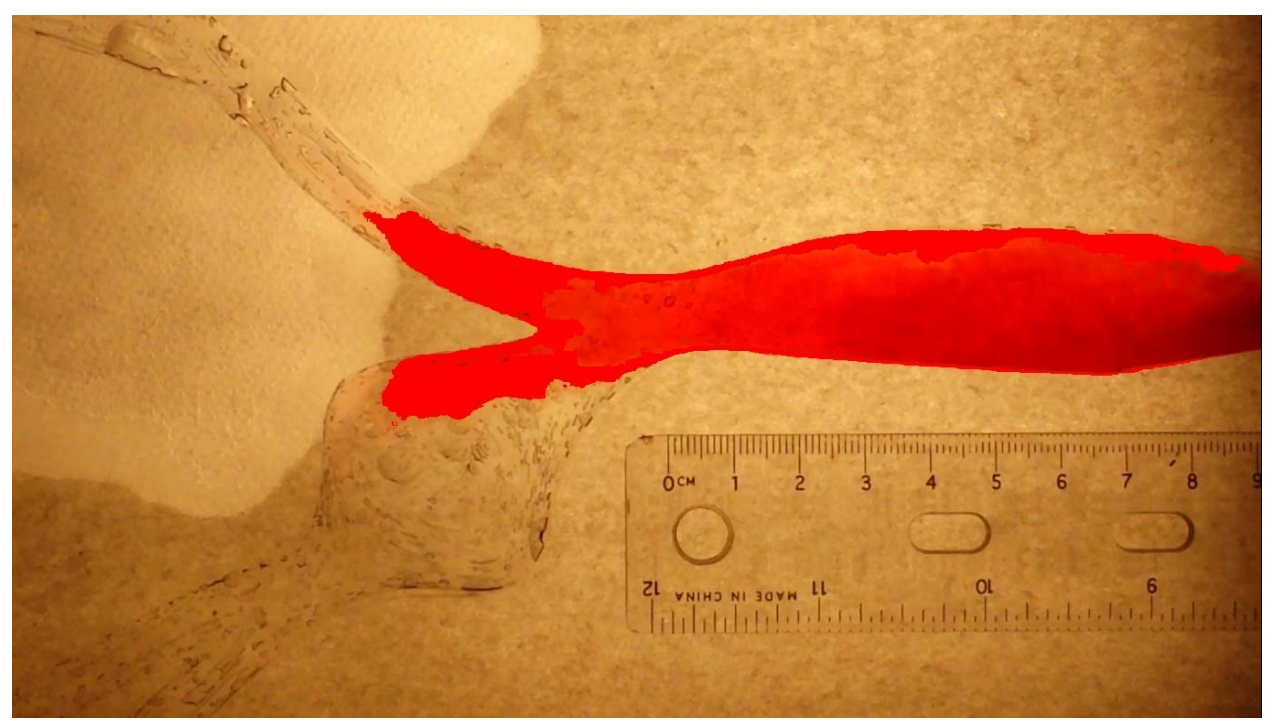

Figure 83: Run 6, Frame 6. 


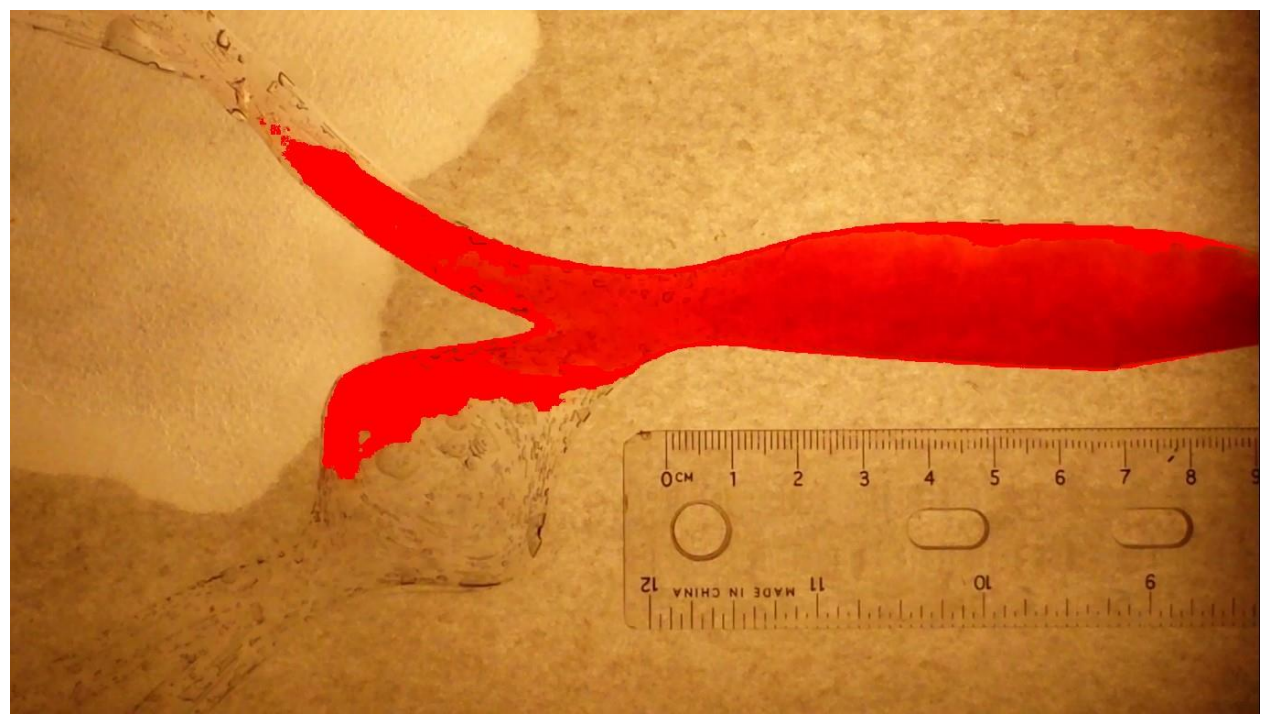

Figure 84: Run 6, Frame 7.

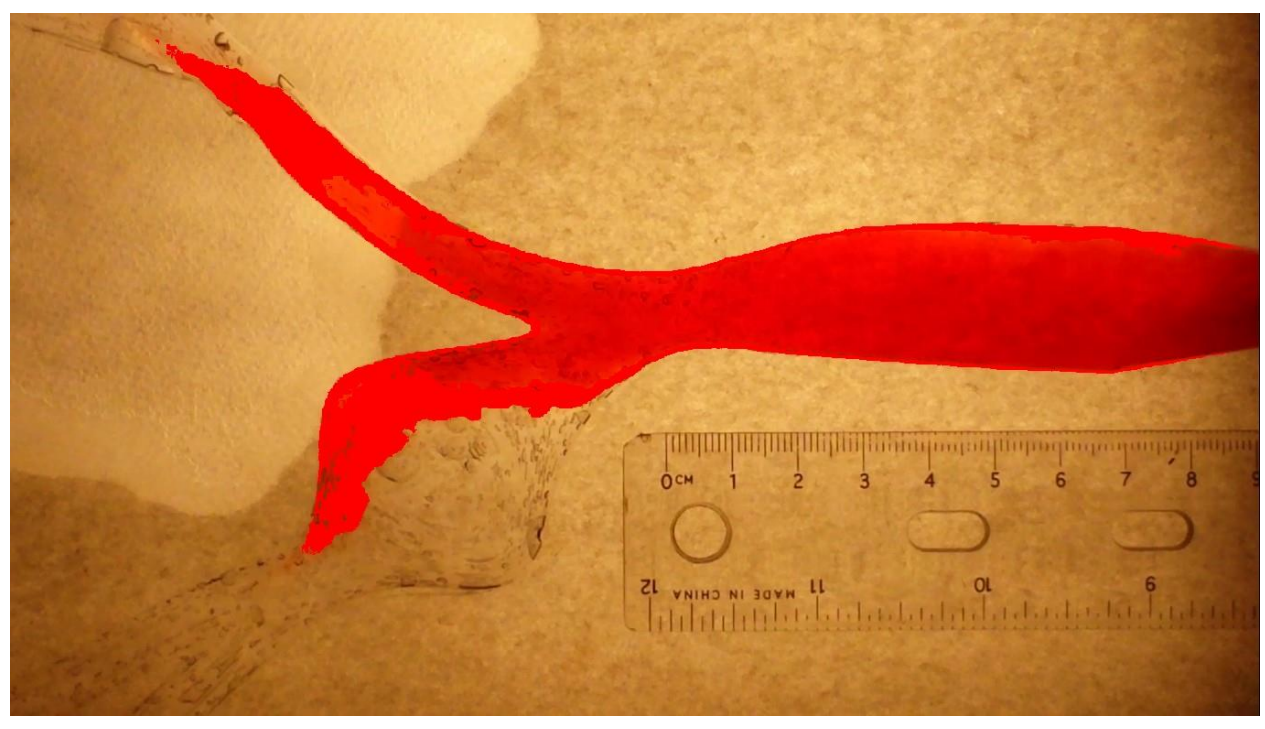

Figure 85: Run 6, Frame 8. 


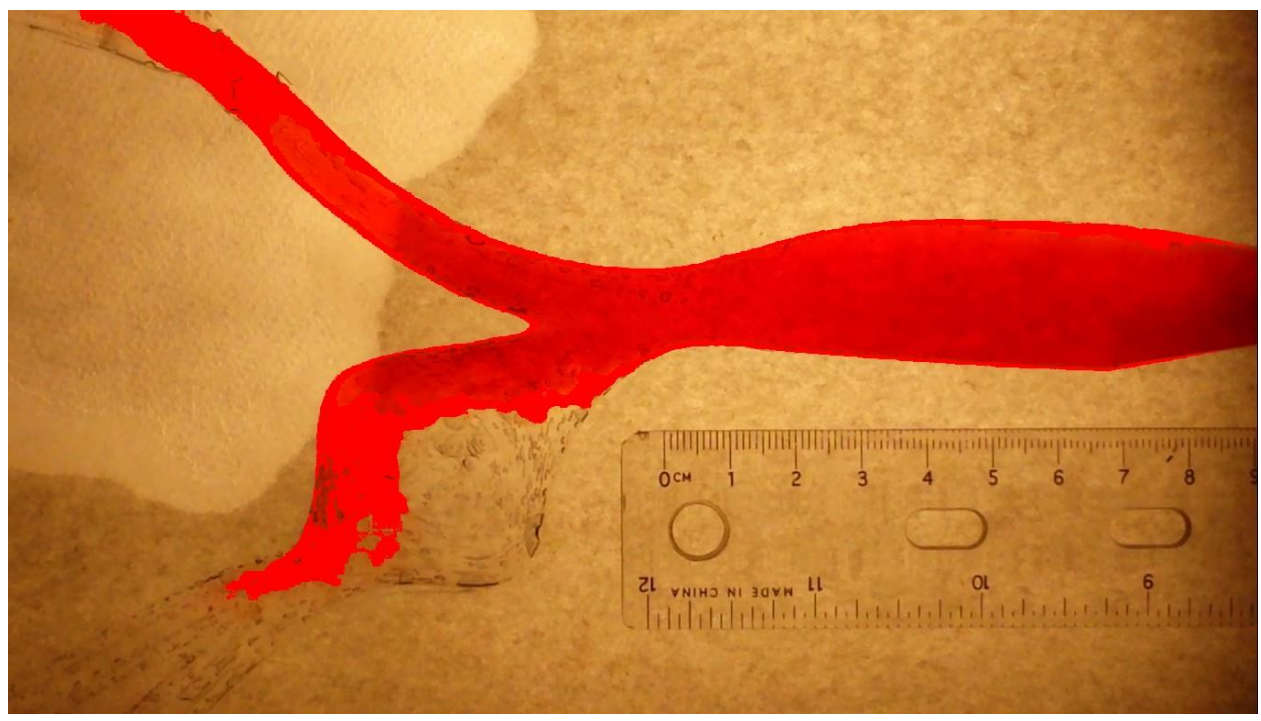

Figure 86: Run 6, Frame 9. 